MARCELO RASSY TEIXEIRA

UMA CONTRIBUIÇÃO PARA A MODELAGEM NUMÉRICA DA HETEROGENEIDADE DO CONCRETO COM O MÉTODO DE GALERKIN LIVRE DE ELEMENTOS 


\section{UMA CONTRIBUIÇÃO PARA A MODELAGEM NUMÉRICA DA HETEROGENEIDADE DO CONCRETO COM O MÉTODO DE GALERKIN LIVRE DE ELEMENTOS}

Tese apresentada à Escola Politécnica da Universidade de São Paulo, como parte dos requisitos para obtenção do título de Doutor em Engenharia de Estruturas.

Área de Concentração:

Engenharia de Estruturas

Orientador:

Prof. Dr. -Ing. hab. Paulo de Mattos Pimenta 
Este exemplar foi revisado e alterado em relação à versão original, sob responsabilidade única do autor e com a anuência de seu orientador.

São Paulo, de janeiro de 2012.

Assinatura do autor

Assinatura do orientador

FICHA CATALOGRÁFICA

Teixeira, Marcelo Rassy

Uma contribuição para a modelagem numérica da heterogeneidade do concreto com o método de Galerkin livre de elementos / M.R. Teixeira. -- ed.rev. -- São Paulo, 2012.

$115 \mathrm{p}$.

Tese (Doutorado ) - Escola Politécnica da Universidade de São Paulo. Departamento de Engenharia de Estruturas e Geotecnica.

1. Concreto 2. Método sem malha 3. Micromecânica 4. Mecânica do dano I. Universidade de São Paulo. Escola Politécnica. Departamento de Engenharia de Estruturas e Geotécnica II. t. 
Esse trabalho é dedicado ao meu filho Raulino, que me ensinou o verdadeiro significado da vida: ser feliz. 


\section{AGRADECIMENTOS}

- Agradeço a Deus, por nunca ter me abandonado nesta longa jornada de trabalho. Estando presente nos diversos momentos difíceis, pessoais e profissionais, que passei ao longo desses cinco anos. Por ter me apoiado quando achei que não encontraria solução. Por ter me ajudado a encontrar forças e a superar as grandes barreiras da vida que se colocaram diante de mim.

- Agradeço a minha esposa Aléta, meu filho Raulino, minhas irmãs Maria e Marcela, meu irmão Marcio, meu afilhado Otávio, meu sobrinho(a) que ainda está no ventre da minha irmã Maria, minha sogra Luzia, cunhados, tios, tias, primos e primas. Eu tenho muito orgulho da minha família.

- Agradeço em especial ao meu pai, Raulino (in memorian) e a minha mãe, Terezinha. Infelizmente Deus já levou meu pai desta jornada terrena. Ele era meu melhor amigo e foram devidos seus ensinamentos me tornei o que sou hoje. E minha mãe, devido seu imenso amor e carinho, incansável em proporcionar o bem estar dos seus filhos, me tornei uma pessoa com bom coração.

- Agradeço aos integrantes do Centro John Argyris: meu orientador Prof. Pimenta, Jorge, Eduardo, Fernando, Leonardo, Paulo, Cintia, Alexandre, Henrique, Evandro, Campello e Liliana. Eu resumo tudo escrevendo que julgo que criamos uma grande família, onde vai ficar os laços por muito tempo. Assim como também agradeço as grandes amizades que conquistei na EPUSP em especial Luis, Igor, Márcia, Tarsis, Plinio e Ricardo.

- Agradeço aos meus grandes amigos da UFPA/CAMTUC que acreditaram e me apoiaram para conseguir fazer meu Doutorado, em especial cito algumas dessas pessoas: Malaquias, Cleison, Carolina, Aarão e Francisco.

- Agradeço a UFPA/CAMTUC e ao CNPq, pelo apoio financeiro. Sem esse apoio se tornaria impossível cursar meu doutorado em São Paulo. 
“A grande luta da vida é contra seus próprios limites.”

Autor desconhecido 


\section{RESUMO}

TEIXEIRA, M.R. Uma contribuição para a modelagem numérica da heterogeneidade do concreto com o método de Galerkin livre de elementos. 2011. 110f. Tese (Doutorado) Escola Politécnica, Universidade de São Paulo, São Paulo, 2011.

Este trabalho apresenta uma metodologia de análise da heterogeneidade do concreto a partir de modelos computacionais desenvolvidos com o método de Galerkin livre de elementos. Esse método se caracteriza pela discretização de um domínio de interesse por um conjunto de partículas sem que exista explicitamente uma malha de elementos no sentido convencional. O objetivo é a previsão das propriedades mecânicas macroscópicas do material resultante a partir das fases individuais e do arranjo geométrico. O concreto foi admitido, na escala mesoscópica, como um composto formado por inclusões (agregado graúdo) imersas em uma matriz (argamassa). Para a simulação foi desenvolvida uma formulação multiregiões onde se admitiu que cada agregado e a argamassa são domínios distintos interligados nas suas interfaces. Para isto foram utilizadas técnicas de subdivisões do domínio (elemento representativo) ao ponto que os seus comportamentos mecânicos não foram comprometidos. Para simular o processo das perdas de rigidez com a formação da fissuração no concreto foi admitido o efeito da mecânica do dano contínuo através do modelo de Mazars. Para as análises foram desenvolvidos modelos computacionais bidimensionais e tridimensionais da heterogeneidade do concreto. A geometria dos agregados foi aproximada por circunferências e elipses no caso 2D e por esferas e elipsoides no caso 3D. Como conclusão a metodologia de multiregiões com o método de Galerkin livre de elementos foi satisfatória e os modelos apresentaram caminhos preferencias de ruptura adequados durante a evolução da danificação.

Palavras-chaves: Concreto; Método de Galerkin livre de elementos; Elemento representativo; Homogeneização; Modelo de Mazars 


\begin{abstract}
TEIXEIRA, M.R. A contribution to the numerical modeling of the heterogeneity of concrete with the element free Galerkin method. 2011. 110f. Thesis (Doctoral) Polytechnic School, University of Sao Paulo, Sao Paulo, 2011.
\end{abstract}

This thesis presents a methodology for analyzing the heterogeneity of concrete from computational models developed with the element free Galerkin method. This method is characterized by discretization of a domain of interest by a set of particles with no explicit mesh in the conventional sense. The goal is to predict the macroscopic mechanical properties of the material resulting from the individual phases and the geometric arrangement. The concrete was assumed, in the mesoscopic scale, as a compound formed by inclusions (coarse aggregate) embedded in a matrix (mortar). For the simulation, a formulation was developed where multi regions were admitted, assuming that each aggregate and mortar are distinct domains connected by their interfaces. For this we used techniques of subdivisions of the domain (representative elements) to the point that their mechanical behaviors were not compromised. To simulate the process of loss of stiffness with the formation of cracks in the concrete, continuum damage mechanics was admitted through Mazars' model. For the analysis, two-dimensional and three-dimensional computer models of the heterogeneity of the concrete were developed. The shape of the aggregates was approximated by circles and ellipses in the two-dimensional case, and by spheres and ellipsoids for the 3D problems. In conclusion the multi region methodology with the element free Galerkin methods was satisfactory and the models presented suitable preferred paths for the rupture during the evolution of damage.

Keywords: Concrete; Element free Galerkin method; Micromechanics; Representative elements; Homogenization; Mazars’ models. 


\section{LISTA DE FIGURAS}

Figura 1 - Localização no Brasil da (a) UHE Tucuruí e (b) UHE Itaipu................................... 4

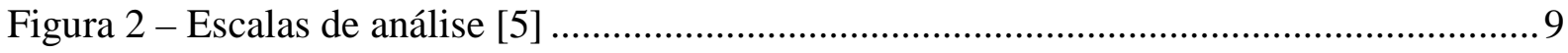

Figura 3 - Heterogeneidade do Concreto [1] ..................................................................... 11

Figura 4 - (a) obtenção do RVE e (b) obtenção do RUC ........................................................... 13

Figura 5 - Célula representativa para um material heterogêneo com duas fases características. [42]........ 15

Figura 6 - Precisão dos resultados em função a proporção do tamanho do RVE. [32] ........... 18

Figura 7 - Processo de danificação do material quase-frágil. [69] .......................................... 24

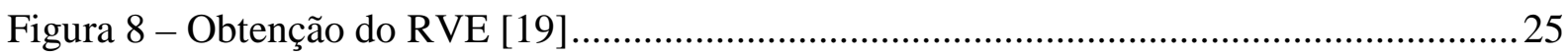

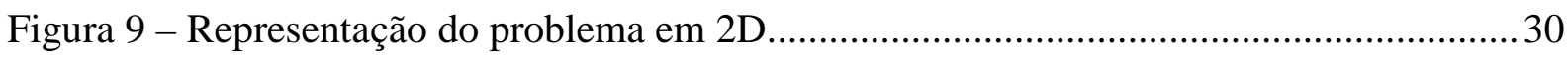

Figura 10 - (a) Discretização pelo MGLE com nuvem de partículas e (b) Discretização pelo

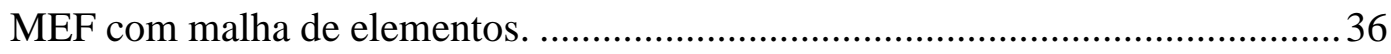

Figura 11 - Método dos Mínimos Quadrados Móveis ........................................................... 40

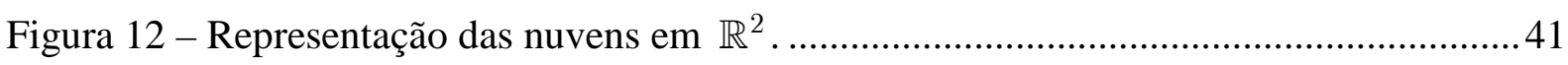

Figura 13 - Distribuição de partículas (a) na matriz (b) no agregado.................................... 44

Figura 14 - Discretização da Fronteira Essencial em Elementos Finitos................................. 45

Figura 15 - Distribuição de partículas na interface. (a) no espaço paramétrico (b) no espaço

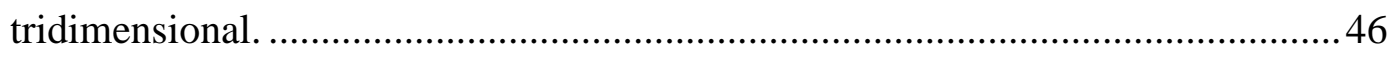

Figura 16 - (a) Concreto (b) Aproximação para círculos e elipses da geometria dos agregados

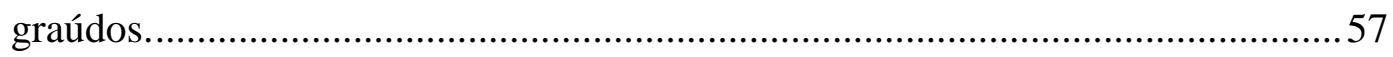

Figura 17 - Esquema do ensaio de compressão axial. ......................................................... 58

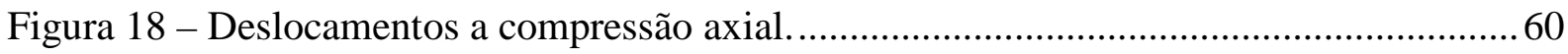

Figura 19 - (a) Nuvem com 400 partículas, (b) Nuvem com 3600 partículas, (c) Deslocamentos verticais com a nuvem de 400 partículas e (d) Deslocamentos verticais com a nuvem de 3600 partículas.......................................................... 61

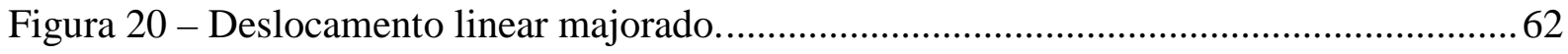

Figura 21 - Deslocamentos verticais. (a) e (b) Inclusão no domínio, (c) e (d) Inclusão na

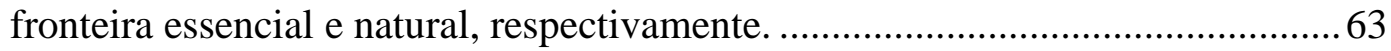

Figura 22 - (a) Deslocamentos verticais com duas inclusões e (b) Deslocamentos verticais

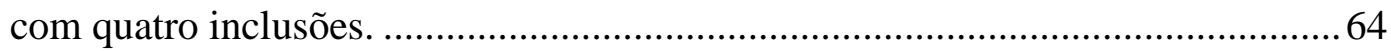


Figura 23 - Obtenção da modelo com várias inclusões.

Figura 24 - Detalhe do modelo com várias inclusões.

Figura 25 - Deslocamentos verticais

Figura 26 - (a) Distribuição das tensões verticais e (b) Distribuição das deformações verticais 66

Figura 27 - Comportamento do material homogeneizado. 67

Figura 28 - Comportamento do módulo de elasticidade homogeneizado...... 69

Figura 29 - Evolução da danificação do modelo com uma inclusão. ...................................... 70

Figura 30 - Processo de ruptura esperado para o modelo com uma inclusão. [5] ................... 71

Figura 31 - Redivisão dos elementos representativos no corpo de prova............................... 71

Figura 32 - Evolução da danificação no modelo com sete inclusões. .................................... 72

Figura 33 - Convergência com matriz elástica e matriz danificada....................................... 73

Figura 34 - Evolução da rigidez do material com incremento de deslocamento...................... 75

Figura 35 - (a) Modelo 3D com uma inclusão e (b) Nuvem de partículas refinadas na interfase

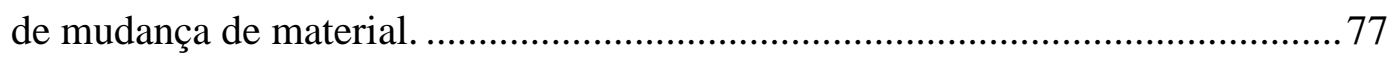

Figura 36 - Deslocamentos do modelo com uma inclusão esférica....................................... 77

Figura 37 - Deformações do modelo com uma inclusão esférica. ......................................... 78

Figura 38 - Tensões do modelo com uma inclusão esférica. ................................................. 78

Figura 39 - Tensões na interface da inclusão esférica. ......................................................... 79

Figura 40 - Modelo com inclusão de 13 agregados.......................................................... 80

Figura 41 - Deslocamentos no eixo Z do modelo com 13 agregados.................................... 81

Figura 42 - Deslocamentos nos eixos X e Y do modelo com 13 agregados............................81

Figura 43 - Distribuição das deformações no modelo com 13 agregados. ............................... 82

Figura 44 - Distribuição das tensões no modelo com 13 agregados........................................ 83

Figura 45 - Curva do módulo de elasticidade do modelo com várias inclusões e diferentes

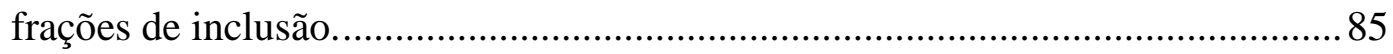

Figura 46 - Croqui esquemático da homogeneização da rigidez do concreto. ........................ 87

Figura 47 - (a) Modelo com a região de danificação, (b) Região danificada obtida na literatura

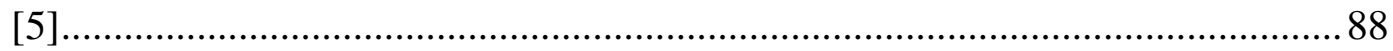

Figura 48 - Caminhos preferencias de ruptura do concreto.............................................. 88 


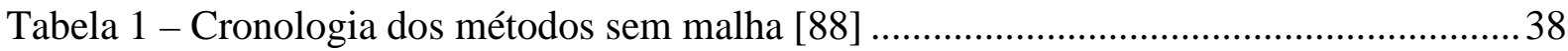

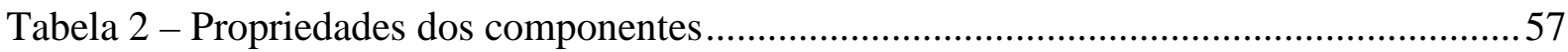

Tabela 3 - Parâmetros do modelo constitutivo de Mazars ........................................................ 57

Tabela 4 - Resultados obtidos no processo de homogeneização ............................................. 68

Tabela 5 - Homogeneização do Material com a evolução do dano ........................................ 74

Tabela 6 - Descrição das propriedades do modelo ................................................................... 77

Tabela 7 - Homogeneização das propriedades elásticas do modelo com uma inclusão esférica 83

Tabela 8- Homogeneização das propriedades do modelo com várias inclusões e com diferentes frações de inclusões. ............................................................................. 84 


\section{SUMÁRIO}

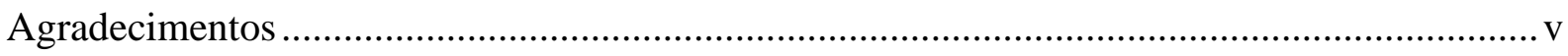

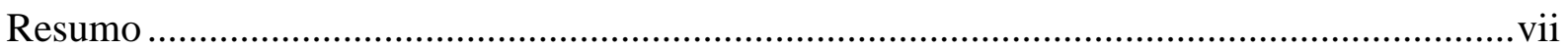

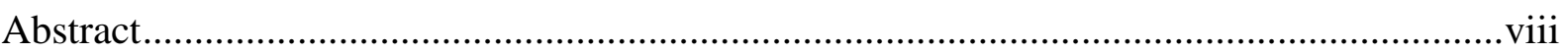

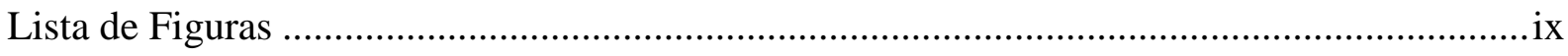

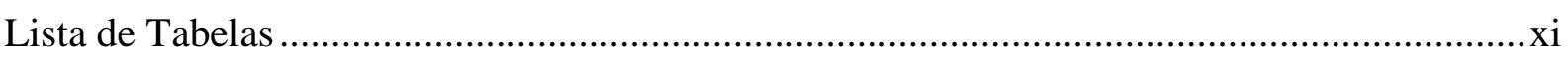

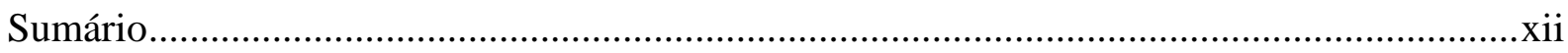

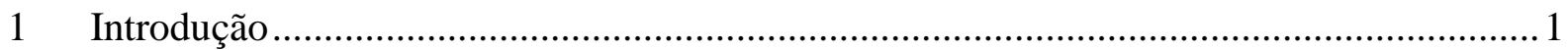

1.1 Relevância do Tema Proposto.................................................................2

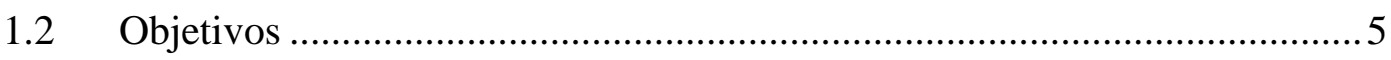

1.2.1 Objetivos Gerais ............................................................................ 5

1.2.2 Objetivos Específicos .................................................................

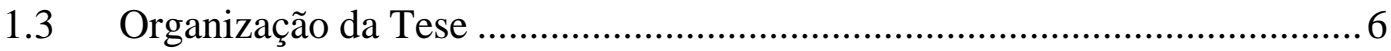

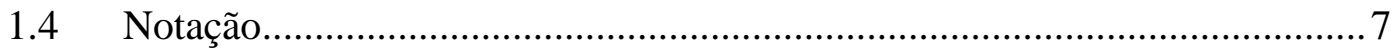

2 Fundamentos da Mesomecânica do Concreto ...................................................................

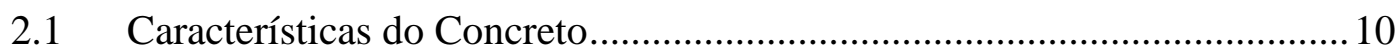

2.2 Definição do Elemento de Volume Representativo (RVE) ........................ 11

2.2.1 Homogeneização das Propriedades Elásticas ........................................ 15

2.2.2 Lema de Hill .................................................................................... 17

2.3 Homogeneização Equivalente de Elsheby Modificada ................................. 18

2.3.1 Método de Mori-Tanaka ....................................................................2 21

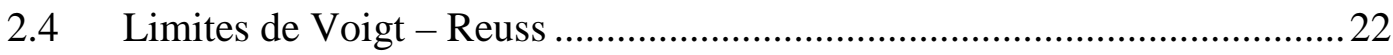

2.5 Modelo de Dano Contínuo ............................................................................ 23

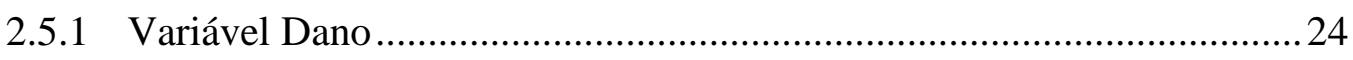

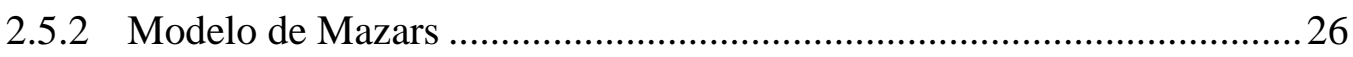

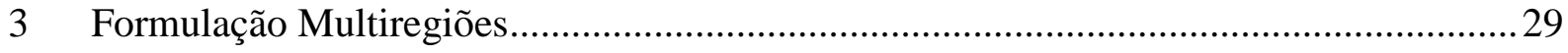

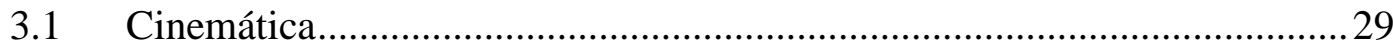

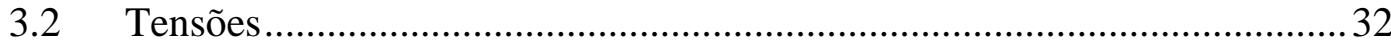

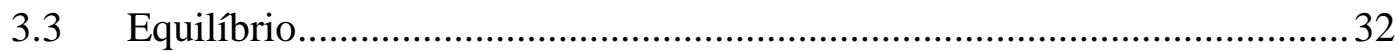

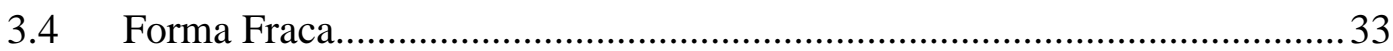


4 Método de Galerkin Livre de Elementos (MGLE) ......................................................... 36

4.1 Aproximação por Mínimos Quadrados Móveis .......................................... 39

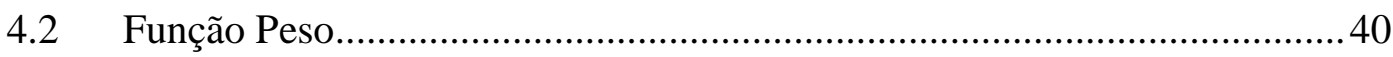

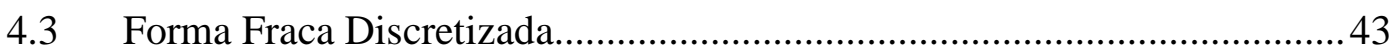

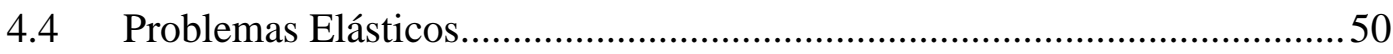

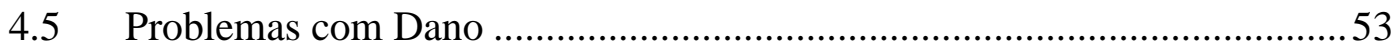

5 Experimentos Númericos Bi-Dimensionais ….............................................................. 57

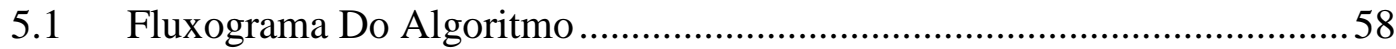

5.2 Modelo De Material Heterogêneo Com Uma Inclusão.................................60

5.3 Modelo De Material Heterogêneo Com Várias Inclusões ...........................63

5.4 Homogeneização Dos Modelos Heterogêneos...........................................67

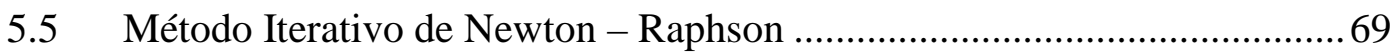

5.5.1 Evolução Do Dano No Elemento Representativo ..................................69

5.5.2 Convergência Nas Iterações ................................................................ 73

5.5.3 Homogeneização Dos Modelos Com Dano............................................ 73

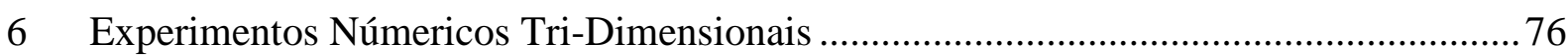

6.1 Modelo De Material Heterogêneo Com Uma Inclusão................................ 76

6.2 Modelo De Material Heterogêneo Com Várias Inclusões .......................... 79

6.3 Homogeneização Dos Modelos Heterogêneos........................................... 83

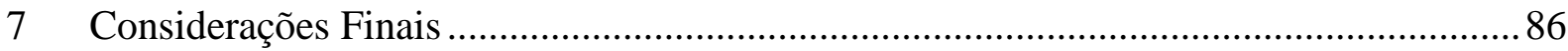

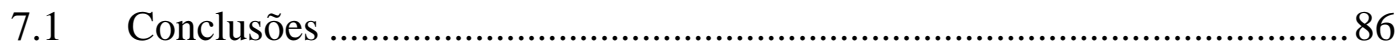

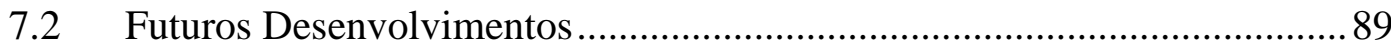

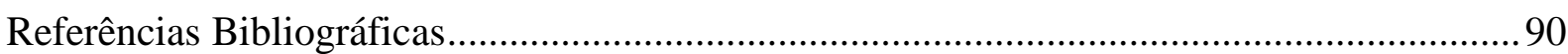




\section{INTRODUÇÃO}

O concreto é um material poroso, com uma estrutura bastante heterogênea e complexa. Analisando a sua mesoestrutura $\left(\approx 10^{-3} \mathrm{~m}\right)$ identificam-se dois constituintes: a pasta de cimento endurecida e as partículas de agregado graúdo. Segundo METHA \& MONTEIRO [1]:

\footnotetext{
"Cada uma das fases do concreto é de natureza multifásica. Toda partícula de agregado pode conter vários minerais, além de microfissuras e vazios. Analogamente, tanto a matriz da pasta como a zona de transição contém geralmente uma distribuição heterogênea, de diferentes tipos e quantidades de fases sólidas, poros e microfissuras, acrescentando-se ainda o fato de estarem sujeitas a modificações com o tempo, umidade ambiente e temperatura, o que torna o concreto, diferentemente de outros materiais de engenharia, um material com características parcialmente intrínsecas ao material.”
}

Atualmente, o ramo da engenharia que está em ampla expansão e cada vez se tornando mais importante é a análise do envelhecimento de estruturas. O desenvolvimento de técnicas para conhecer a mesoestrutura do concreto presente nas estruturas e o nível de deterioração que a mesma teve durante os anos de utilização é o que precisa para poder estimar o tempo restante da vida útil dessas estruturas. Um exemplo clássico desta atividade é a análise de segurança de usinas hidrelétricas e nucleares com vários anos de utilização. Este ramo é altamente multidisciplinar, contando com engenheiros especialistas em estruturas, construção e materiais [2].

O elemento de volume representativo (RVE) do material desempenha um papel central no desenvolvimento da mecânica de materiais heterogêneos, a fim de prever as suas propriedades de forma eficaz. DRUGAN \& WILLIS [3] propuseram que o RVE seja o elemento com menor volume do material heterogêneo para a qual apresentam boas respostas das constantes constitutivas quando comparadas com as obtidas na macroestrutura. O RVE é um método de homogeneização que apresenta resultados satisfatórios especialmente nos casos de propriedades lineares como é o caso das características elásticas do material.

Nesta pesquisa foi utilizado para os processamentos numéricos dos modelos um workstation com dois processadores Xeon modelo X5650 de seis núcleos de processamento 
cada, quatro discos rígidos de um terabyte em "RAID10” e quarenta e oito gigabyte de memória RAM.

\subsection{Relevância do Tema Proposto}

A vida útil do concreto depende da qualidade dos materiais utilizados, do grau de agressividade ambiental e dos tipos de carregamentos. Porém como comumente é um material projetado para ter uma vida útil longa, e grandes obras serem constituídas predominantemente por este material, o governo e a sociedade não imaginam perder construções marcantes provocadas pela deterioração do material. Com isso, desenvolveram-se modelos computacionais que simulam o comportamento deste material admitindo a sua heterogeneidade e como evolui a danificação ao longo do seu domínio.

A fundação nacional de ciências e tecnologia dos Estados Unidos emitiu um relatório denominado SBES (Blue Ribbon Panel on Simulation-Based Engineering Science) [4] no ano de 2006 com o apoio de diversos pesquisadores renomados mundialmente, onde relataram que a área de ciência e tecnologia tem como uma das metas o desenvolvimento dos materiais.

Um dos maiores impactos na sociedade é justamente nas inovações metodológicas da modelagem e simulação dos materiais. A modelagem multiescala transforma-se com o desenvolvimento de novos materiais e/ou o aperfeiçoamento dos materiais já existentes. Esta transformação é equivalente a uma mudança para um novo paradigma. Os benefícios do desenvolvimento de novos materiais são amplamente evidentes no progresso atual nas áreas da nanociência, da tecnologia e da bioengenharia. [4]

Para a manutenção do concreto precisam-se desenvolver técnicas de restauro e/ou previsões do seu real estado de conservação. As previsões podem ser feitas através de monitorações com sensores e sistema de aquisição de dados, ou através do desenvolvimento de modelos computacionais que simulem esses comportamentos. A segunda opção é predominantemente mais viável, segundo dois aspectos: econômico e tempo de resposta.

Outra motivação é o pioneirismo deste estudo. Atualmente pesquisadores vêm se aprofundando no desenvolvimento de modelos computacionais que simulem o 
comportamento mesomecânico de materiais como concreto, aço, fibra de carbono, borracha, nono-tubo de carbono, etc. Porém, ainda não se chegou a um modelo constitutivo que simule propriamente o comportamento mesomecânico do concreto. E no Brasil, ainda tem muito que fazer para o desenvolvimento destes modelos.

A relevância deste assunto se explica também devido cada vez mais ser necessário o entendimento do comportamento dos materiais presentes em obras marcantes da sociedade, como por exemplo, as usinas hidrelétricas. Alguns exemplos, usina hidrelétrica de Tucuruí, usina hidrelétrica de Itaipu, Ponte Otávio Frias de Oliveira e altos fornos de siderúrgicas, serão descritos para um melhor entendimento da importância do assunto.

- A usina hidrelétrica de Tucuruí (UHE Tucuruí) é a maior usina genuinamente brasileira, localizada a $350 \mathrm{~km}$ da capital Belém, estado do Pará. Foi construída para a geração de energia elétrica e para tornar navegável um trecho do rio Tocantins cheio de corredeiras, ultrapassadas através de duas eclusas interligadas por um canal. Em números a UHE Tucuruí é responsável pela geração de 11,960 MW e 7,919 milhões de metros cúbicos de concreto. A UHE Tucuruí é responsável por cerca de 15\% da energia consumida no Brasil.

- A usina hidrelétrica de Itaipu (UHE Itaipu) é uma usina binacional localizada no rio Paraná, na fronteira entre o Brasil e o Paraguai. Fica localizada a 640 km da capital Curitiba, estado do Paraná. Para sua construção foram necessários 12,57 milhões de metros cúbicos de concreto. Hoje é responsável por 20\% da energia consumida no Brasil. A Figura 1ilustra a localização no território brasileiro das duas principais usinas hidrelétricas responsáveis por quase $40 \%$ da energia total consumida no Brasil. 


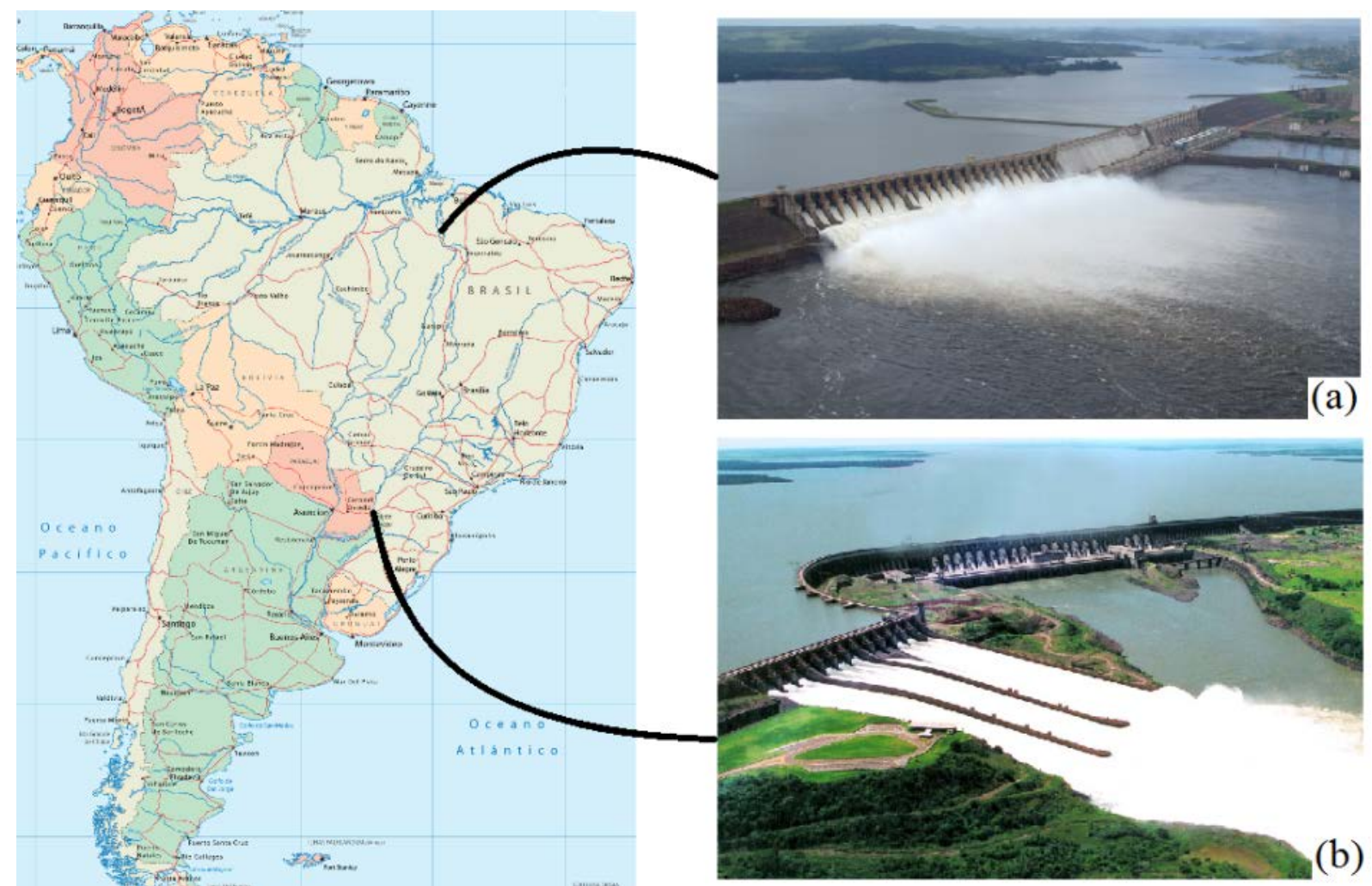

Figura 1 - Localização no Brasil da (a) UHE Tucuruí e (b) UHE Itaipu.

- A ponte Octávio Frias de Oliveira que faz parte do complexo viário real parque, é formada por duas pistas estaiadas em curva independentes de $60^{\circ}$ que cruzam o rio Pinheiros, no bairro do Brooklin, na cidade de São Paulo. Foi inaugurada em maio de 2008, após três anos de construção. Erguida em concreto protendido, as alças foram moldadas por meio de formas deslizantes. A obra consumiu aproximadamente 58.700 metros cúbicos de concreto.

- Nas siderúrgicas, se faz necessário, o entendimento do comportamento do concreto presente nos alto-fornos de cozimento. Esses fornos têm estruturas de concreto armado revestidas internamente por concretos isolantes e materiais refratários. Além de suportar o peso próprio desses materiais, a estrutura de concreto deve suportar também o carregamento mecânico cíclico provocado pelo processo térmico de cozimento dos materiais em questão. $\mathrm{O}$ exemplo disso, está nos fornos de cozimento de anodos presentes no processo de fabricação do alumínio primário, que comumente apresentam deformação lenta nas paredes externas de concreto armado provocado pelo ciclo a cada 18 
dias de aquecimento e resfriamento interno. Os custos desses fornos giram em torno de cinquenta milhões de dólares.

\subsection{Objetivos}

O objetivo desta pesquisa é o desenvolvimento de modelos avançados da mesoestrutura do concreto, admitindo a sua heterogeneidade com as inclusões dos agregados no seu domínio. Para isto, foi utilizado o método de Galerkin livre de elementos, por ser um método sem malha, não precisando assim que em toda mudança de material seja muito refinada, como é necessário no caso do método dos elementos finitos, que utiliza malhas de elementos, precisando um grande refinamento na interface agregado - argamassa.

Esta pesquisa é a parte introdutória de um grande projeto a ser desenvolvido, que tem por objetivo a simulação computacional do envelhecimento do concreto de barragens. A idéia será a obtenção do real estado de conservação das principais barragens de concretos presentes nas usinas hidrelétricas no Brasil. A exemplo disso, citam-se as duas principais que são a UHE Tucuruí e a UHE Itaipu que juntas são responsáveis por quase $40 \%$ da energia consumida no território brasileiro.

Com o estudo da evolução do dano, pode-se notar de forma qualitativa que os comportamentos das estruturas de concreto no nível macro são reflexos dos diversos fatores que incidem na microestrutura do concreto. Ressalta-se que o fraturamento do material na escala macro se origina de uma evolução da danificação na mesoescala.

\subsubsection{Objetivos Gerais}

Um melhor entendimento do comportamento mecânico linear e não linear do concreto com a utilização do método de Galerkin livre de elementos, através de modelos computacionais que admitem a sua heterogeneidade. 


\subsubsection{Objetivos Específicos}

- O desenvolvimento de modelos da mesomecânica do concreto com o método de Galerkin livre de elementos para facilitar uma definição mais detalhada das interfaces entre os diferentes materiais. O método de Galerkin livre de elementos se enquadra nos métodos sem malha, onde em vez de criar uma malha de elementos, utiliza uma nuvem de partículas para definir o domínio do sólido.

- Um melhor entendimento do comportamento mecânico do concreto quando submetido a carregamentos crescentes, admitindo nos modelos computacionais como sendo um material heterogêneo, na mesoescala, com a presença de inclusões circulares e elípticas para os casos bidimensionais e esféricas e elipsoidais para os casos tridimensionais.

- Desenvolvimento da evolução do dano no meio contínuo do concreto com o incremento de carregamento, através do uso do modelo de dano contínuo de Mazars. Nessa evolução pode se comparar com o que está definido na literatura do comportamento de um modelo com apenas uma inclusão.

- Obtenção da homogeneização do material a partir de análises lineares e não lineares quando admitida a heterogeneidade do concreto. Com isso pode se verificar que os modelos computacionais de estrutura do concreto, quando admitida a heterogeneidade do material, apresentaram resultados mais realísticos quando comparados com modelos que admitem o concreto como material homogêneo.

\subsection{Organização da Tese}

Além deste capítulo introdutório, a tese está dividida em seis capítulos. O capítulo 2 relata uma revisão do que já foi desenvolvido na literatura sobre o assunto proposto. No capítulo 3 é apresentado a formulação multiregiões. No capítulo 4 descreve-se o método de Galerkin livre de elementos, sendo o método numérico utilizado nesta pesquisa. Nos capítulos 5 e 6 expõem-se os resultados obtidos nos diversos modelos numéricos. No capítulo 7 as conclusões e considerações finais. Em detalhe, a Tese apresenta: 
- Capítulo 2 introduz os fundamentos da mesomecânica do concreto, as características do concreto, a definição do elemento representativo, a homogeneização das propriedades elásticas, o lema de Hill, a homogeneização equivalente de Eshelby modificado, o método de MoriTanaka, os limites de Reuss - Voigt, o método de dano contínuo, a variável dano e o modelo de Mazars.

- Capítulo 3 apresenta a formulação multiregiões, a cinemática do problema, as tensões, o equilíbrio e a forma fraca. Essa formulação foi a diferencial na metodologia para a discretização da heterogeneidade do material nos modelos computacionais.

- Capítulo 4 apresenta a metodologia do método de Galerkin livre de elementos, as aproximações por mínimos quadrados móveis, a função peso, forma fraca discretizada, a formulação dos problemas elásticos e dos problemas com dano.

- Capítulo 5 e 6 são apresentados os experimentos numéricos bi- e tridimensionais, respectivamente. No desenvolvimento da metodologia é apresentado o fluxograma do algoritmo, modelo de materiais heterogêneos com uma inclusão, modelo de materiais heterogêneos com várias inclusões, a homogeneização dos modelos heterogêneos, a evolução do dano nos modelos através do método incremental-iterativo de Newton - Raphson e a homogeneização dos modelos danificados.

- Capítulo 7 contém as considerações finais, onde são apresentadas a síntese dos resultados e as conclusões obtidas. Em seguida se propõem futuros desenvolvimentos como continuação desta pesquisa.

\subsection{Notação}

Ao decorrer do texto, adota-se:

(1) Letras minúsculas itálicas, gregas ou latinas $(a, b, \ldots, \alpha, \beta, \ldots)$ representam escalares.

(2) Letras minúsculas itálicas e negritas, gregas ou latinas $(\boldsymbol{a}, \boldsymbol{b}, \ldots, \boldsymbol{\alpha}, \boldsymbol{\beta}, \ldots)$ representam vetores. 
(3) Letras maiúsculas itálicas e negritas, gregas ou latinas $(\boldsymbol{A}, \boldsymbol{B}, \ldots)$ representam tensores de segunda ordem no espaço vetorial Euclidiano.

(4) A convenção da somatória de Einstein para índices repetidos é adotada, com índices gregos variando de 1 a 2 e índices latinos de 1 a 3 . 


\section{FUNDAMENTOS DA MESOMECÂNICA DO CONCRETO}

As análises para a determinação das propriedades macroscópicas de materiais compostos é um problema clássico da ciência e da engenharia, atraindo a atenção de celebridades como Maxwell e Einstein. A atenção atual é focada no desenvolvimento das análises do comportamento dos materiais heterogêneos [5] nas escalas nanoscópia, microscópica, mesoscópica e macroscópica. Na mesoescala (Figura 2) assume-se uma escala com dimensões maiores que as das moleculares, de modo que o domínio possui propriedades macroscópicas, mas muito menor que a dimensão macroscópica.

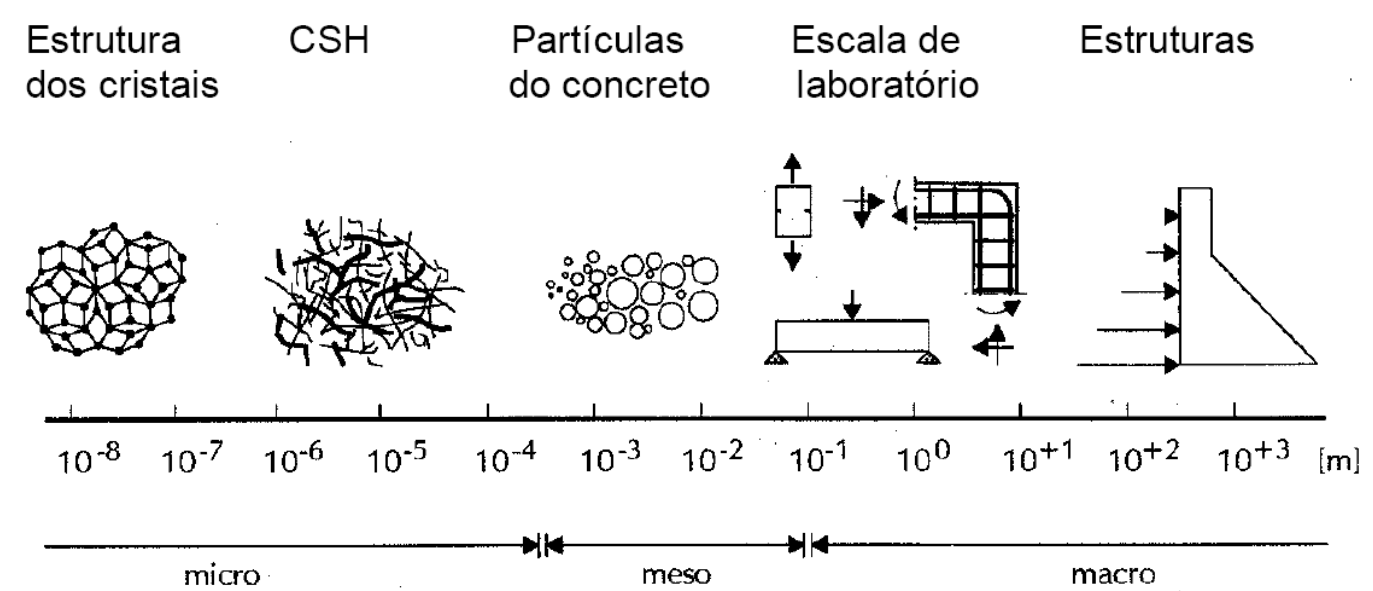

Figura 2 - Escalas de análise [5]

Um material heterogêneo é aquele que é composto por diferentes fases, tais como os compósitos, ou mesmo materiais em diferentes estados, tais como os policristais. O concreto pode ser um meio contínuo quando analisado macroscopicamente ou na escala mesoscópica, diversos grãos (agregado graúdo) imersos em um meio comum (argamassa). Para obtenção das propriedades efetivas deste material tem-se com a premissa básica a obtenção das frações das diferentes fases presentes no mesmo. [6] [7] [8] [9]

O estudo da mesoestrutura do material está associado na obtenção das propriedades efetivas do mesmo divididas em quatro classes: tensor de condutividade efetivo, tensor de elasticidade efetiva, tensor de permeabilidade efetiva e vida útil efetiva do material. [10] [11] [12] [13] [14] [15] [16] [17] [18] 
Em razão dos fenômenos de expansão e retração da matriz quando do processo de endurecimento do concreto, pode-se admitir uma terceira fase formada ao redor dos grãos. Essa fase, denominada de zona de transição, se desenvolve em condições de ainda baixa resistência da matriz, dando margem à criação de vazios e defeitos de aderência. Assim, a existência de vazios na estrutura matricial ou mesmo a geração dos citados defeitos durante o processo de fabricação do concreto permitem afirmar que esse material sempre apresentará uma dada heterogeneidade. [19] [20]

\subsection{Características do Concreto}

Basicamente o concreto tem três razões principais que justificam a sua grande utilização:

(1) a sua excelente resistência a água,

(2) a facilidade com a qual elementos estruturais de concreto podem ser obtidos através de uma variedade de formas e tamanhos,

(3) e sua rápida disponibilidade do material para o uso.

A aplicação de modelos mesomecânicos é para a obtenção das propriedades mecânicas macroscópicas a partir desta escala. Estes modelos fornecem as equações constitutivas que podem ser utilizadas para simular o comportamento de elementos estruturais. Essas técnicas também permitem a análise dos fenômenos locais tais como a iniciação e acumulação de danos nos compósitos durante a deformação. [21]

O tipo, a quantidade, o tamanho, a forma e a distribuição das fases presentes em um sólido constituem a sua mesoestrutura. A partir da investigação de uma seção transversal do concreto (Figura 3), as fases que podem ser distinguidas são os agregados graúdos com formas e tamanhos variados e o meio ligante composto de uma massa de pasta de cimento hidratada. 


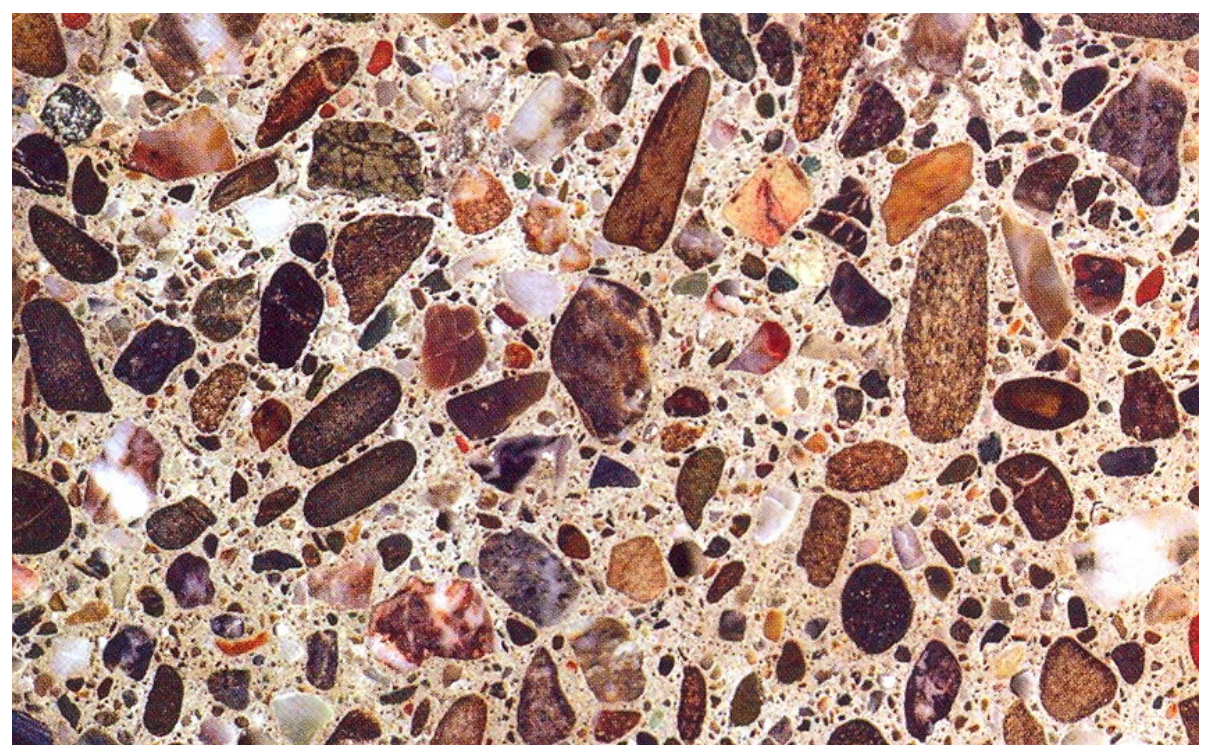

Figura 3 - Heterogeneidade do Concreto [1]

Quando o concreto é submetido a um carregamento ao longo do tempo, primeiramente nota-se uma deformação instantânea, a qual é seguida por um acréscimo de deformação no decorrer do tempo chamado de fluência. Além disso, submetido ou não a carregamento, o concreto se contrai quando perde umidade, sofrendo uma retração.

As intensidades da retração e da fluência são da mesma ordem de grandeza da deformação elástica devida a tensões usuais, de modo que os diversos tipos de deformações sempre devem ser levados em conta. Percebe-se, na verdade, que as deformações nas estruturas reais de concreto, que frequentemente levam à fissuração, ocorrem devido à resposta do material a cargas externas, ao material empregado, à forma da estrutura e ao meio ambiente. [22]

\subsection{Definição do Elemento de Volume Representativo (RVE)}

A modelagem computacional de materiais está se tornando uma ferramenta confiável para as pesquisas científicas e para complementação de tradicionais abordagens teóricas e experimentais. Nas análises multiescala (contínua, quando visto na macroescala e mesoescala, e discreta quando visto na escala atômica) a compreensão da estrutura do material apresenta interdependências necessárias para as análises. 
A modelagem multiescala é um termo com aplicabilidade geral, mesmo que restrita ao campo de materiais compósitos. Em seu sentido amplo pode ser descrita como atravessar todas as escalas de comprimento a partir dos átomos, até estruturas de engenharia com tamanhos representativos. As ferramentas de previsão aplicáveis nas modelagens multiescala podem usar desde a mecânica quântica, a mecânica estatística e as abordagens clássicas de Engenharia baseadas na elasticidade linear e não-linear elastoplástica do material. Nesta pesquisa utilizaram-se as abordagens clássicas. [23]

As estimativas das propriedades mecânicas efetivas dos materiais multifásicos são de interesse para pesquisadores e engenheiros em muitas áreas da Engenharia. As chamadas propriedades efetivas de um composto heterogêneo são obtidas no volume com as médias ao longo de um elemento de volume representativo e que caracteriza uma escala mesoscópica.

O concreto é tratado pela maioria dos modelos apenas tendo uma fase homogênea. Isso resulta em previsões imprecisas do comportamento deste material sob condições de carregamento. Neste trabalho, o concreto é considerado como um material compósito formado por dois componentes: argamassa e agregado graúdo. Na mesoescala esses componentes formam uma matriz contínua, com a inclusão dos agregados graúdos. O comportamento não linear ocorre através da danificação com a evolução das microfissuras ao logo do domínio do material. [24]

Os desempenhos dos concretos são afetados pelas tensões que atuam na interface entre a pasta endurecida de cimento (ou argamassa) e o agregado graúdo [25]. Assim nesta linha de trabalho na mesoescala, os experimentalistas têm observado um aumento progressivo das tensões de ruptura com o surgimento de microfissuras nestas regiões. Os modelos mesomecânicos podem prever com precisão o comportamento do concreto. Desta forma vem sendo desenvolvido modelos que simulem as microfissuras que se localizam na camada entre a argamassa e os agregados graúdos. [26]

O objetivo da mesomecânica é a previsão das propriedades mecânicas macroscópicas de materiais heterogêneos a partir das fases individuais e do arranjo geométrico. Análises mesomecânicas são normalmente conduzidas ao conceito de elementos representativos do volume (RVE) e/ou a células unitárias periódicas (RUC). O RVE e RUC são técnicas de 
subdivisões do domínio do material ao ponto que os seus comportamentos mecânicos não são comprometidos. Para o caso do concreto que apresenta agregados graúdos dispersos aleatoriamente no volume do concreto será utilizado neste estudo o conceito do RVE. A figura 4 ilustra a diferença entre essas duas técnicas de obtenção de um elemento na escala mesomecânica.

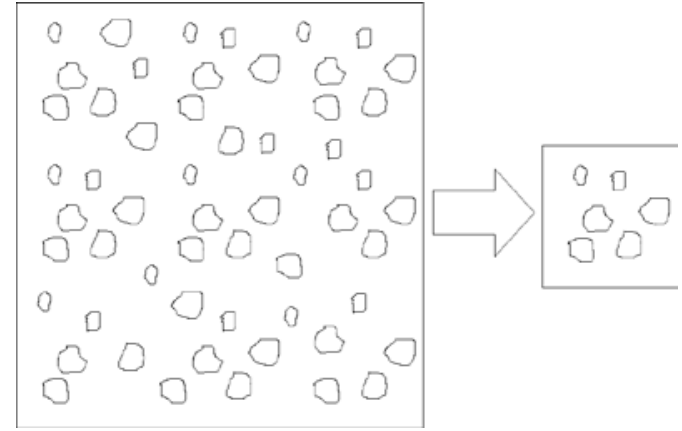

(a)

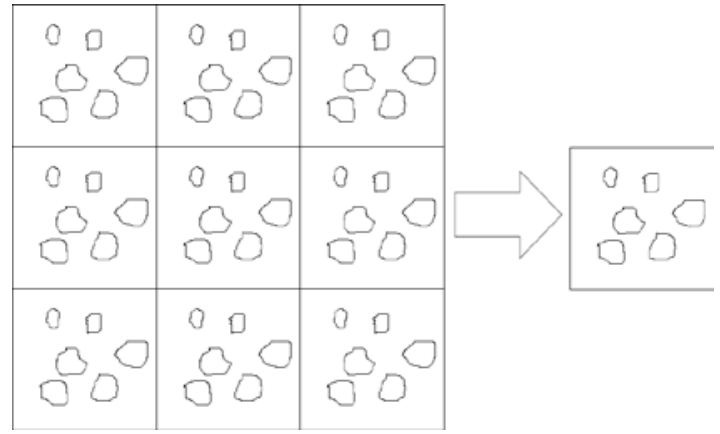

(b)

Figura 4 - (a) obtenção do RVE e (b) obtenção do RUC

A definição do tamanho do RVE não é uma tarefa fácil e não é conhecida a priori. Para o caso com uma distribuição aleatória da mesoestrutura, deve ser assumido um RVE fictício, porém com significativa resposta estatística. Evidentemente ao gerar um RVE fictício todas as informações disponíveis da mesoestrutura devem ser consideradas. As mesoestruturas representativas são apropriadas para um RVE, porém há muitas discussões sobre qual tamanho mínimo deve ser considerado. Nos casos em que se obtêm mesoestruturas regulares podendo-se obter o RUC, os resultados obtidos são considerados mais representativos. [27] [28] [29] [30] [31] [32] [33] [34]

A etapa atual sobre o uso dessas análises em estruturas com materiais compostos está na obtenção eficaz dos módulos, dos deslocamentos locais, dos campos de tensões para a previsão das forças. O cálculo dos campos de tensões em nível meso para a homogeneização do material continua sendo o principal alvo das investigações científicas. [35]

HUET [36] [37] introduziu o conceito de módulo aparente obtido a partir de repetidas análises mesomecânicas de materiais com diferentes tamanhos de RVE e sob diferentes condições de contorno. A questão sobre o número de inclusões contidas no RVE tem sido o tema principal das investigações. 
HOLLISTER \& KIKUCHI [38] [39] abordaram quantitativamente a diferença entre o RVE e o RUC em materiais heterogêneos com inclusões cilíndricas, onde se verificou a necessidade de aumentar o RVE quando submetido a um grande número de RUC. PECULLAN et. al. [40] estenderam a pesquisa considerando agora, diferentes módulos de rigidez para as inclusões do compósito, apresentando resultados melhores para uma fração baixa de inclusões.

KANIT [41] propôs uma metodologia para obtenção da célula representativa de um material heterogêneo. A pesquisa desenvolveu a análise numérica e então foi obtida a estatística dos resultados. Para a determinação do RVE de uma microestrutura, pode-se:

- Gerar diferentes microestruturas com 4 ou 5 tamanhos.

- Registrar as propriedades aparentes obtidas em cada microestrutura.

- Calcular o valor médio e o desvio padrão e avaliar se os números de microestruturas foram suficientes para definir o tamanho.

- Definir a precisão que se queira para a estimativa das propriedades efetivas do material e com isso definir o número de microestruturas e o tamanho da célula representativa.

O erro é obtido segundo a fórmula:

$$
\text { erro }=\frac{2 D_{z}(V)}{\sqrt{n}}
$$

onde $D_{z}(V)$ é a variação dos dados das propriedades coletadas do RVE e $n$ é o número de amostras de cada RVE. A Figura 5 ilustra a obtenção dos resultados das propriedades efetivas em função do tamanho do RVE. 


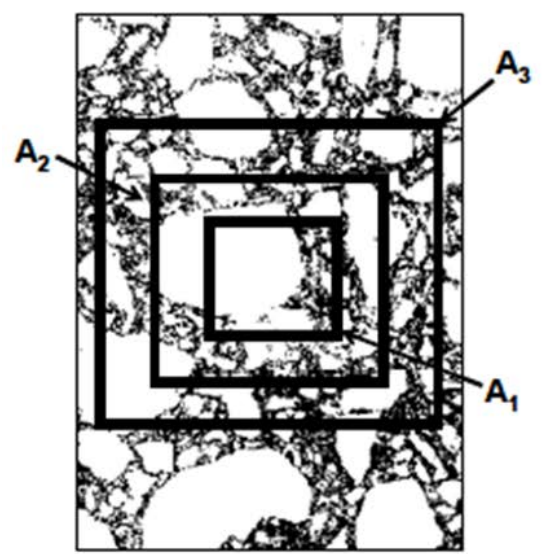

Figura 5 - Célula representativa para um material heterogêneo com duas fases características. [42]

SWAMINATHAN [43] [44] desenvolveu a equivalente célula representativa da mesoestrutura estatisticamente (SERVE) com o objetivo de obter um RVE com propriedades efetivas dos compósitos com variações de concentração das partículas e variação da dispersão. Seguindo esse conceito, POVIRK [45]determinou o tamanho do RVE a partir da estatística da mesoestrutura com duas fases aleatórias, obtendo como resultado, que são necessários para respostas satisfatórias, RVE com dimensões suficientes para obter apenas doze inclusões. Inclusão é um subdomínio presente no sólido e que apresenta propriedades distintas quando comparado com a matriz. [30]

\subsubsection{Homogeneização das Propriedades Elásticas}

A determinação macroscópica das propriedades efetivas dos materiais heterogêneos depende do modelo geométrico escolhido para o material investigado e, portanto, o tipo de condições de contorno aplicadas no RVE. A deformação efetiva e a tensão efetiva são os nomes para descrever a equivalência com a deformação e a tensão, respectivamente, encontrada na célula representativa da mesoestrutura. Um material heterogêneo é estatisticamente homogêneo na escala apropriada quando se caracteriza pelos módulos de elasticidade que não variam ponto a ponto nessa escala.

O conceito dos módulos de elasticidade de materiais heterogêneos é baseado nos módulos de elasticidades dos materiais homogêneos que os compõem e que devem ser independentes da forma de aplicação das condições de contorno. Os módulos de elasticidade 
efetiva são definidas no menor elemento representativo do material cuja média das respostas são representados pela resposta do material como um todo. Pode-se definir para o RVE as tensões e deformações médias

$$
\langle\boldsymbol{\sigma}\rangle=\frac{1}{V} \int_{V} \boldsymbol{\sigma}(\boldsymbol{x}) d V
$$

e

$$
\langle\varepsilon\rangle=\frac{1}{V} \int_{V} \varepsilon(\boldsymbol{x}) d V
$$

respectivamente, e $V$ é o volume do RVE.

A associação do tensor na escala meso com tensor na escala macro é chamado de homogeneização. A teoria da homogeneização lida com um problema de distribuição de massa e pode ser considerado como um problema de síntese de material. Nestes métodos baseados na teoria da homogeneização, busca-se uma representação consistente do ponto de vista mecânico, fazendo-se uso das leis básicas de mistura e das condições de periodicidade dos meios.

Para os modelos bi-dimensionais foram admitidos estado plano de tensões, material isotrópico, notação de Voigt e a partir das tensões e deformações efetivas, chegou-se a:

$$
\nu=\frac{\left\langle\sigma_{y}\right\rangle\left\langle\varepsilon_{x}\right\rangle-\left\langle\sigma_{x}\right\rangle\left\langle\varepsilon_{y}\right\rangle}{\left\langle\sigma_{x}\right\rangle\left\langle\varepsilon_{x}\right\rangle-\left\langle\sigma_{y}\right\rangle\left\langle\varepsilon_{y}\right\rangle}
$$

e

$$
E=\frac{\left\langle\sigma_{x}^{2}\right\rangle-\left\langle\sigma_{y}^{2}\right\rangle}{\left\langle\sigma_{x}\right\rangle\left\langle\varepsilon_{x}\right\rangle-\left\langle\sigma_{y}\right\rangle\left\langle\varepsilon_{y}\right\rangle},
$$


onde $\nu$ e $E$ são os coeficiente de Poisson e o módulo de elasticidade do material homogeneizado e as tensões e deformações aparecem segundo a notação de engenharia.

Para os modelos tri-dimensionais, o material foi homogeneizado através do módulo volumétrico $(K)$ e do módulo de elasticidade transversal $(G)$, resultando em WRIGGERS [34] [33] :

$$
3 K=\frac{\frac{\operatorname{tr}\langle\boldsymbol{\sigma}\rangle}{3}}{\frac{\operatorname{tr}\langle\varepsilon\rangle}{3}}
$$

e

$$
2 G=\left(\frac{\left\langle\boldsymbol{\sigma}^{\prime}\right\rangle:\left\langle\boldsymbol{\sigma}^{\prime}\right\rangle}{\left\langle\boldsymbol{\varepsilon}^{\prime}\right\rangle:\left\langle\boldsymbol{\varepsilon}^{\prime}\right\rangle}\right)^{1 / 2},
$$

onde $K$ e $G$ são os módulo volumétrico e o módulo de elasticidade transversal do material homogeneizado. Para se obter o coeficiente de Poisson e o módulo de elasticidade homogeneizado, utilizam-se as relações

$$
G=\frac{E}{2(1+\nu)}
$$

e

$$
K=\frac{E}{3(1+\nu)}
$$

\subsubsection{Lema de Hill}

Dada a tensão $\boldsymbol{\sigma}$ compatível com a deformação $\varepsilon(\varepsilon=\varepsilon(\boldsymbol{u}))$, e cada tensão e deformação satisfazendo as condições de contorno, tem-se o teorema de Hill: 


$$
\langle\sigma: \varepsilon\rangle=\langle\sigma\rangle:\langle\varepsilon\rangle
$$

Esse teorema define a igualdade entre a média de $\sigma: \varepsilon$ microscópico - trabalho devido às tensões na escala microscópica $-\mathrm{e}\langle\boldsymbol{\sigma}\rangle:\langle\varepsilon\rangle$, trabalho devido às tensões médias, macroscópico. A figura 6 ilustra qualitativamente a precisão dos resultados em função da proporção do tamanho do RVE.

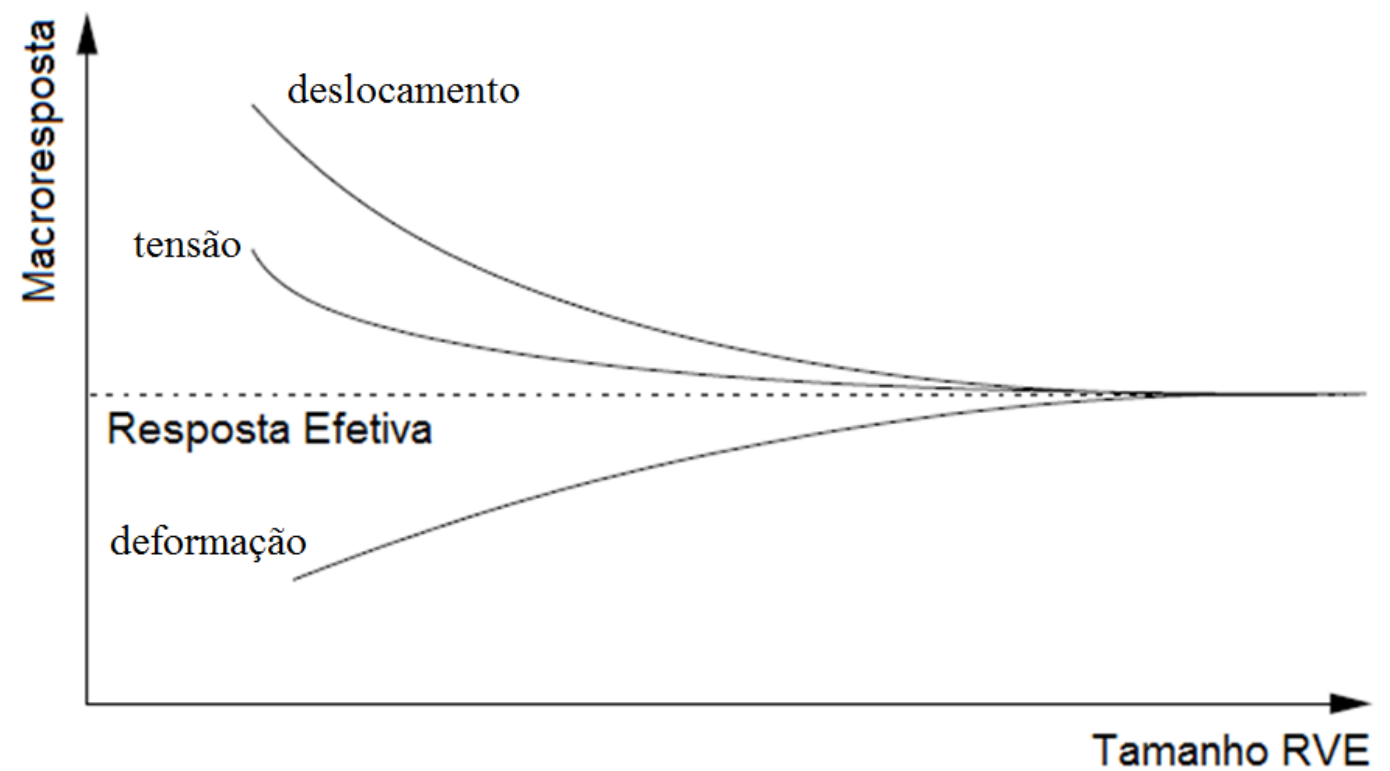

Figura 6 - Precisão dos resultados em função a proporção do tamanho do RVE. [32]

\subsection{Homogeneização Equivalente de Elsheby Modificada}

A distribuição de tensões entre duas fases em um compósito pode ser obtida pelo método de Eshelby. Para isto, é necessário admitir uma perfeita ligação na interface inclusãomatriz, além de considerar a ausência de trincas e de porosidade assumida ao longo deste desenvolvimento.

A distribuição não uniforme de tensões pode surgir, durante o experimento, por causa das diferenças entre as constantes elásticas da matriz e da inclusão. A equação da tensão pode ser expressa, mais simplesmente, onde na maioria dos materiais de interesse, $C$ é a matriz de elasticidade. 
O método de Eshelby consiste em extrair uma região da matriz de mesma constante elástica, isotrópica infinita, a qual será chamada de inclusão equivalente. Em seguida, ela é imaginada como submetida a uma ampliação livre de tensões $\varepsilon^{T}$, a qual será chamada de deformação ou forma transformada, sem mudança de constante elástica.

Uma pressão superficial é usada para fazer essa região retornar ao tamanho original, de maneira que se possa recolocá-la no furo formado na matriz no momento em que ela foi extraída. Ao se retornar esta pressão, o equilíbrio é alcançado entre a matriz e a inclusão, produzindo uma forma contraída. Por isso, essa região será chamada de inclusão equivalente $\varepsilon^{C}$, que tem a forma contraída. Neste caso, além da deformação na inclusão equivalente, aparecerá uma deformação na matriz. A tensão na inclusão, pela lei de Hooke, pode ser expressa em termos de deformação elástica por:

$$
\boldsymbol{\sigma}=C_{M}\left(\varepsilon^{C}-\varepsilon^{T}\right)
$$

ESHELBY [46] [47] [48] relacionou a forma contraída com a forma transformada por meio de um tensor chamado de tensor de Eshelby

$$
\varepsilon^{C}=\boldsymbol{S} \varepsilon^{T}
$$

Quando se estuda um caso real, o processo é o mesmo, porém como a inclusão real é mais rígida que a inclusão equivalente, para haver esta equivalência, é preciso que a forma transformada da inclusão real $\left(\varepsilon^{T^{*}}\right)$ seja menor que a forma transformada da inclusão equivalente.

$$
\varepsilon^{C}>\boldsymbol{S} \varepsilon^{T^{*}}
$$

Assim, podemos expressar

$$
\boldsymbol{\sigma}_{I}=\boldsymbol{C}_{M}[\boldsymbol{S}-\boldsymbol{I}]\left[\left(\boldsymbol{C}_{I}-\boldsymbol{C}_{M}\right) \boldsymbol{S}+\boldsymbol{C}_{M}\right]^{-1} \boldsymbol{C}_{I} \varepsilon^{T^{*}}
$$

onde: 
$C_{M}$ - módulo de elasticidade da matriz.

$C_{I}$ - módulo de elasticidade da inclusão.

$S$ - tensor de Eshelby

$I$ - tensor identidade

O tensor de Eshelby só depende da geometria e das constantes elásticas do material. Já existem expressões para seus componentes para os muitos casos de interesse (elipsoides, fibras longas, círculos,...). [49] [50] [51] [52] [53] [54] [55] [56] [57] [58] [59] [60] [61]. Este tensor é de quarta ordem que apresenta as seguintes propriedades:

- Apresenta simetria com os respectivos dois primeiros índices e os dois últimos índices.

$$
S_{i j k l}=S_{j i k l}=S_{i j \mid k}
$$

Porém, não se pode generalizar a simetria para o caso de $i j$ e $k l$.

$$
S_{i j k l} \neq S_{k l i j}
$$

- As propriedades do material da inclusão independem para o tensor.

- O tensor depende somente dos parâmetros que definem a geometria da inclusão e das propriedades elásticas do material onde foi submetida à inclusão.

- Caso o material do meio que ocorreu a inclusão seja homogêneo, o tensor depende somente do coeficiente de Poisson da matriz e dos parâmetros da geometria da inclusão.

O modelo de Eshelby modificado é utilizado para os casos de experimentos com frações de inclusão elevados, como é o caso do concreto. Neste caso, usa-se o conceito de "back stress" que se baseia no balanço de tensões dado pela equação abaixo:

$$
(1-f)\langle\boldsymbol{\sigma}\rangle_{M}+f\langle\boldsymbol{\sigma}\rangle_{I}=0,
$$


onde $\langle\boldsymbol{\sigma}\rangle_{M}$ corresponde ao "back stress" e é a tensão média desenvolvida na matriz, $\langle\boldsymbol{\sigma}\rangle_{I}$ é a tensão média na inclusão e $f$ é a fração volumétrica da inclusão (agregado graúdo) na matriz (argamassa).

Esta tensão média na inclusão vale $\langle\boldsymbol{\sigma}\rangle_{I}$ em compósitos infinitos e $\langle\boldsymbol{\sigma}\rangle_{I}+\langle\boldsymbol{\sigma}\rangle_{M}$ em um compósito finito.

O balanço de tensões é extremamente útil, pois relaciona o campo de tensões médio na matriz com o campo de tensões médio na inclusão. Este efeito pode ser estendido para outras inclusões ou para um modelo de distribuição espacial aleatória de partículas.

\subsubsection{Método de Mori-Tanaka}

Nos anos 70 MORI \& TANAKA [62] e outros pesquisadores oriundos da área de pesquisa de mesomecânica dos compósitos desenvolveram os modelos matemáticos para compósitos multifásicos aplicando-se o conceito de campo médio microscópico para analisar as propriedades macroscópicas dos materiais. Esse campo de médias consideraria que o corpo contém inclusões (fibras, particulados, poros, fissuras) com mesodeformações específicas.

Para a plena demonstração dessa teoria fez-se uso, entre outros, do estudo do vetor de mesodeformações de Eshelby para avaliação do efeito da forma dos dispersos e de toda a Teoria da Elasticidade de corpos isotrópicos e anisotrópicos. Para ilustração resumida da aplicação, segue a forma proposta por YANG \& HUANG [63], ainda para compósitos bifásicos, mas que já utiliza esse tipo de modelagem.

No caso, foram consideradas as hipóteses de aplicação da lei de Hooke generalizada para materiais isotrópicos e homogêneos tanto para o concreto como para as fases pasta de cimento endurecida e agregado (inclusões). Esta última, ainda, inclusive, considerada como de inclusões esféricas:

$$
\boldsymbol{C}_{c}=\left\{\boldsymbol{C}_{M}^{-1}+V_{p}\left[\left(1-V_{p}\right)\left(\boldsymbol{C}_{p}-\boldsymbol{C}_{M}\right) \boldsymbol{T}-V_{p}\left(\boldsymbol{C}_{M}-\boldsymbol{C}_{p}\right)+\boldsymbol{C}_{M}\right]^{-1}\left(\boldsymbol{C}_{M}-\boldsymbol{C}_{p}\right) \boldsymbol{C}_{M}^{-1}\right\}
$$


onde:

- $C_{c}$ - matriz de rigidez do concreto

- $C_{M}$ - matriz de rigidez da argamassa

- $C_{p}$ - matriz de rigidez do agregado

- $V_{p}$ - fração volumétrica de agregado

- $\quad T$ - tensor de Eshelby

\section{$2.4 \quad$ Limites de Voigt - Reuss}

Com base em um modelo mesomecânico de REUSS - VOIGT [64] é possível estabelecer um limite máximo e mínimo para as propriedades elásticas, como por exemplo, o módulo de elasticidade. Considere um material como sendo a mistura de dois materiais, respectivamente com frações volumétricas de $f_{1}$ e $f_{2}\left(f_{1}=1-f_{2}\right)$.

Considere uma "construção em paralelo", onde os dois materiais estão sujeitos à mesma deformação (modelo de Voigt) e outra “construção em série” onde os dois materiais estão sujeitas ao mesmo esforço (modelo de Reuss). Com base nisso é possível estabelecer propriedades equivalentes destes materiais onde:

Modelo de Voigt: $\quad E_{e q}=f_{1} \cdot E_{1}+f_{2} \cdot E_{2}$

Modelo de Reuss:

$$
E_{e q}=\frac{E_{1} \cdot E_{2}}{\left[\left(1-f_{1}\right) \cdot E_{1}+\left(1-f_{2}\right) \cdot E_{2}\right]}
$$

onde $E_{1}$ e $E_{2}$ são os módulos de elasticidade dos dois materiais.

Estas equações correspondem ao limite superior de Voigt e ao limite inferior de Reuss, respectivamente, para o valor do módulo de elasticidade de um material compósito. No entando, conforme referido por HILL [65], nem a suposição de estado de isodeformação nem isotensão representam condições reais. 
De fato, as trações na interface das lâminas da matriz e do reforço não estão em equilíbrio, segundo o modelo de Voigt, e a interface das lâminas da matriz e do reforço não se consegue manter, segundo o modelo de Reuss. Convém referir que não é aconselhável calcular os coeficientes de Poisson sob estados de isotensão ou de isodeformação. [65]

De um modo geral, se os valores da rigidez dos materiais da matriz e da inclusão forem muito diferentes, os limites de Voigt e Reuss definem um intervalo muito amplo.

\subsection{Modelo de Dano Contínuo}

A mecânica do dano contínuo permite descrever os mesosprocessos heterogêneos envolvidos durante o processo de deformação de materiais na macroescala. Os processos de danificação correspondem a localizações e acumulações de deformações que são de caráter irreversível. Os modelos de dano admitem que as perdas de rigidez e de resistência do material são devidas ao processo de mesofissuração.

O trabalho pioneiro que introduziu o conceito de Dano contínuo foi elaborado por KACHANOV [66] [67] [68]. Este trabalho surgiu do interesse em modelar o efeito da fissuração distribuída na ruptura do tipo frágil, observada em metais, após um período de deformação lenta.

A classificação geral dos materiais que incide sobre o progresso da degradação dentro de sua estrutura é dividido em três grupos:

- Materiais frágeis - é composto por materiais com poucas deformações, por exemplo, o vidro, onde ocorre o aparecimento súbito de descontinuidades no domínio.

- Materiais dúcteis - são materiais que apresentam uma certa deformação antes do aparecimento de descontinuidades, como por exemplo os polímeros e metais.

- Materiais quase-frágeis - presentes entre os dois grupos anteriores, esses materiais apresentam o inicio da deterioração com o surgimento de 
microfissuras. A partir daí, ocorre à evolução da danificação, conforme descrito na Figura 7.

GEERS [69] descreveu de forma sistemática o processo da evolução da danificação, passando para a evolução da fratura, finalizando com o colapso do material.

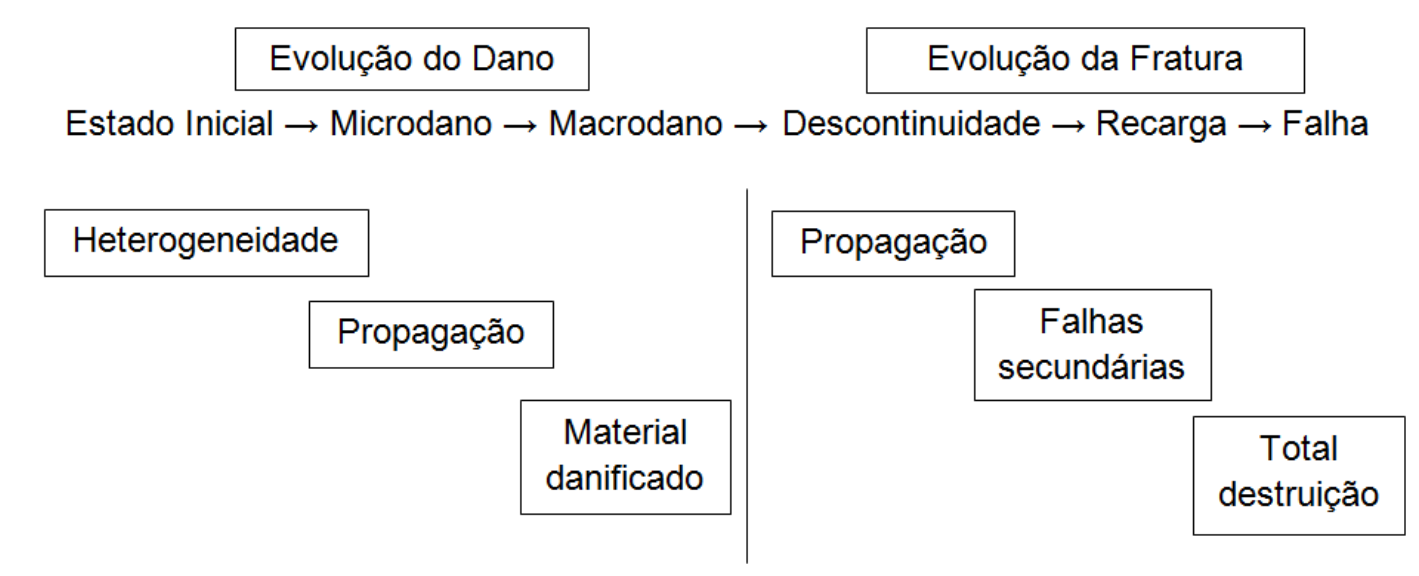

Mecânica do Dano

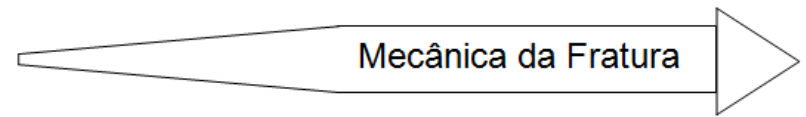

Figura 7 - Processo de danificação do material quase-frágil. [69]

\subsubsection{Variável Dano}

Para auxiliar a definição de uma variável que quantifique o dano, considere o sólido da Figura 8. Tal elemento da figura é dito representativo por possuir dimensão suficientemente grande, de modo que se possa admitir que contenha uma distribuição homogênea dos defeitos e, ao mesmo tempo, é pequeno para ser considerado como um ponto material do contínuo. 


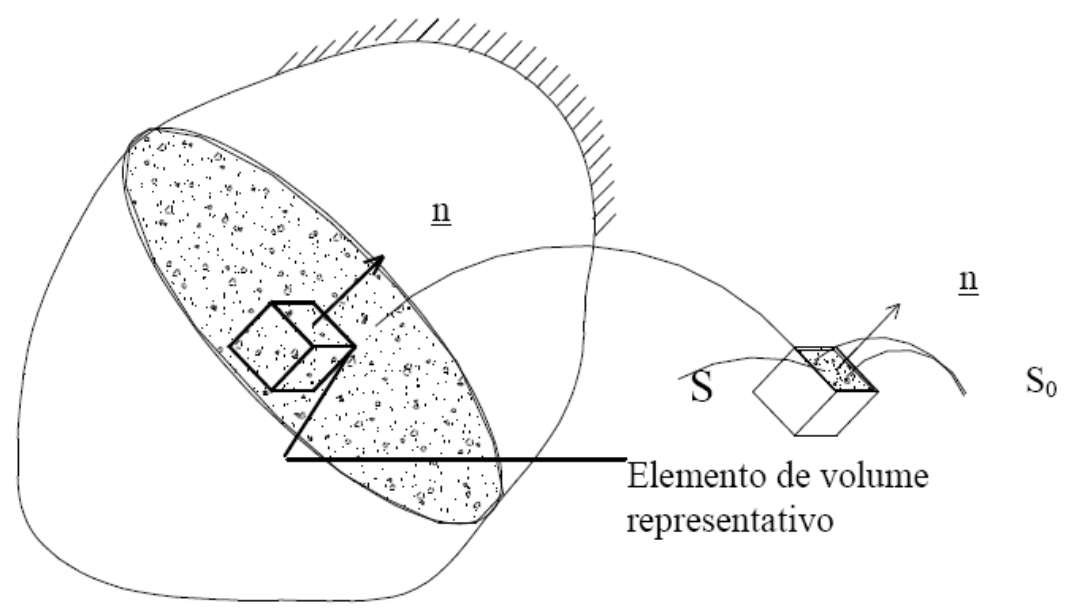

Figura 8 - Obtenção do RVE [19]

$S$ representa a área da seção do elemento e é identificada por sua direção normal $\underline{n}$.

Nesta seção, é suposto existirem trincas que caracterizam o nível de dano. Considerando a área efetiva de resistência $\bar{S}$, sendo $\bar{S}<S$, onde existem trincas, concentradores de tensão e as interações entre os defeitos, pode-se definir a área total danificada como

$$
S_{D}=S-\bar{S}
$$

Pode-se também definir a razão $s_{D} / s=D$ como uma medida mecânica local do dano relativo à direção $\underline{n}$. D representa uma medida local do dano. A variável de dano assume valores contidos no intervalo $0 \leq D \leq 1$, sendo que $D=0$, tem correspondência com a situação de material íntegro e $D=1$, indica um estado de total deterioração.

No caso do dano isotrópico, as trincas e cavidades são orientadas uniformemente em todas as direções. Dessa forma, a variável dano não depende da orientação $\underline{n}$ e o estado danificado é caracterizado por um campo escalar $D$, dependente unicamente da posição no sólido. Esta simplificação numérica, de dano isotrópico, é adotada nesta pesquisa. [67]

Existem algumas linhas de tipos de medidas da variável interna de dano. [67] 
- Medidas em escala de mesoestrutura (densidade de mesofissuras ou cavidades) levando aos modelos mesoscópicos que podem ser integrados sobre o elemento de volume macroscópico, com a ajuda de técnicas matemáticas de homogeneização.

- Medidas físicas globais (densidade, resistividade, etc) requerendo a definição do modelo global para convertê-lo em propriedades que caracterizam a resistência mecânica.

- Avaliação do dano ligado ao tempo de vida restante, mas este conceito não é levado diretamente para a lei constitutiva do dano.

- Medidas mecânicas globais da modificação das propriedades elásticas, plásticas ou viscoplásticas. São medidas fáceis de interpretar utilizando o conceito de tensão efetiva.

Pode-se colocar em evidência a degradação das características mecânicas do material causada pelo dano, mediante a relação que define o módulo de elasticidade $\bar{E}$ para um meio contínuo de resposta equivalente ao meio deteriorado.

$$
\bar{E}=(1-D) E
$$

onde $E$ representa o módulo de elasticidade do meio integro ( $D=0$ ).

\subsubsection{Modelo de Mazars}

O modelo proposto por MAZARS [70] com validade em situações de carregamento aplicado continuamente crescente, tem as seguintes hipóteses fundamentais:

- localmente o dano decorre de deformações de alongamento evidenciadas por sinais positivos, ao menos um deles, das componentes de deformação principal $\left(\varepsilon_{i}>0\right)$;

- o dano é representado por uma variável escalar $D(0 \leq D \leq 1)$ cuja evolução ocorre quando um valor de referência para o alongamento equivalente é superado; 
- o concreto com dano comporta-se como meio elástico. Portanto, deformações permanentes evidenciadas experimentalmente são desprezadas.

Segundo o modelo de Mazars, o dano no concreto inicia quando a deformação normal $\varepsilon_{x x}$ atinge o limite elástico de tração desse material $\varepsilon_{d o}$. A partir desse instante a tensão normal $\sigma_{x}$ passa a se relacionar com a deformação linear específica $\varepsilon_{x x}$ através da seguinte expressão

$$
\sigma_{x}=(1-D) E_{c} \varepsilon_{x x},
$$

sendo $E_{c}$ o módulo de elasticidade do concreto e $D$ a variável de dano escalar, devendo ser observado que enquanto não há dano, essa expressão representa o trecho linear do diagrama tensão-deformação do concreto.

O modelo de Mazars preconiza que o dano dever ser avaliado através de uma combinação de efeitos de tração e compressão, tal como expresso a seguir:

$$
D=\alpha_{T} D_{T}+\alpha_{C} D_{C}
$$

Cabendo observar que, segundo ALVARES [71], para pontos submetidos à:

- Tração axial, $\alpha_{T}=1$ e $\alpha_{C}=0$, resultando em $D=D_{T}$.

- Compressão axial, $\alpha_{T}=0$ e $\alpha_{C}=1$, resultando em $D=D_{C}$.

As parcelas do dano associadas a tração e a compressão são dadas, respectivamente por:

$$
\begin{gathered}
D_{T}\left(\varepsilon_{e q}\right)=1-\frac{\varepsilon_{d o}\left(1-A_{T}\right)}{\varepsilon_{e q}}-\frac{A_{T}}{\exp \left[B_{T}\left(\varepsilon_{e q}-\varepsilon_{d o}\right)\right]} \\
D_{C}\left(\varepsilon_{e q}\right)=1-\frac{\varepsilon_{d o}\left(1-A_{C}\right)}{\varepsilon_{e q}}-\frac{A_{C}}{\exp \left[B_{C} \cdot\left(\varepsilon_{e q}-\varepsilon_{d o}\right)\right]},
\end{gathered}
$$


onde $A_{T}, A_{C}, B_{T}$ e $B_{C}$, são parâmetros experimentais obtidos do diagrama tensão-deformação do material (concreto), $\alpha_{T}, \alpha_{C}$ representam a contribuição de solicitações à tração e à compressão para o estado local de extensão e assumem valores no intervalo fechado [0,1] e $\varepsilon_{e q}$ é a deformação equivalente, como denominada por Mazars, sendo assim expressa:

$$
\varepsilon_{e q} \begin{cases}\varepsilon_{x x} & , \varepsilon_{x x} \geq 0 \\ -\nu \varepsilon_{x x} \sqrt{2} & , \varepsilon_{x x} \leq 0 .\end{cases}
$$

Observando-se que $\nu$ é o coeficiente de Poisson do concreto, a deformação $\varepsilon_{e q}$ é portanto sempre positiva. 


\section{FORMULAÇÃO MULTIREGIÕES}

\subsection{Cinemática}

Seja $\left\{\boldsymbol{e}_{1}, \boldsymbol{e}_{2}, \boldsymbol{e}_{3}\right\}$ uma base ortonormal no espaço, com origem $\boldsymbol{O}=\{0,0,0\}$. Um ponto material pertencente ao corpo é descrito por sua posição $\boldsymbol{x} \in \Omega=x_{1} \boldsymbol{e}_{1}+x_{2} \boldsymbol{e}_{2}+x_{3} \boldsymbol{e}_{3}$. Nos casos bidimensionais, supõe-se também que a superfície representativa do corpo esteja no plano $e_{1} \times e_{2}$, ou seja, $x_{3}=0, \boldsymbol{x}=x_{1} \boldsymbol{e}_{1}+x_{2} \boldsymbol{e}_{2}$.

Um corpo heterogêneo é descrito como a união das regiões que o formam. No presente caso, uma região será a matriz e cada agregado constituirá outra.

$$
\Omega=\Omega_{1} \cup \Omega_{2} \cup \cdots \cup \Omega_{\text {nreg }},
$$

onde $n r e g$ é o número de regiões do problema (o número de agregados mais um). A fronteira do problema é dada por $\Gamma=\partial \Omega=\Gamma_{u} \cup \Gamma_{t}$, onde $\Gamma_{u}$ é a parte da fronteira onde deslocamentos são impostos (condições essenciais de contorno) e $\Gamma_{t}$ é a parte da mesma onde tensões são impostas (condições naturais de contorno). Estas também estão divididas de acordo com a região adjacente:

$$
\begin{gathered}
\Gamma_{u}=\Gamma_{u-1} \cup \Gamma_{u-2} \cup \cdots \cup \Gamma_{u-n r e g}, \\
\Gamma_{t}=\Gamma_{t-1} \cup \Gamma_{t-2} \cup \cdots \cup \Gamma_{t-n r e g} .
\end{gathered}
$$

Define-se também a fronteira de interface entre as regiões como a superfície de contato entre materiais distintos. Para maior simplicidade na notação no presente trabalho, recorre-se à suposição que dois agregados não se interceptam. Logo, todas as fronteiras de interface $\Gamma_{i}^{a}, a=2$..nreg são entre um agregado e a matriz. Estas regiões e superfícies, para o caso 2D, podem ser vistas na Figura 9 abaixo. 


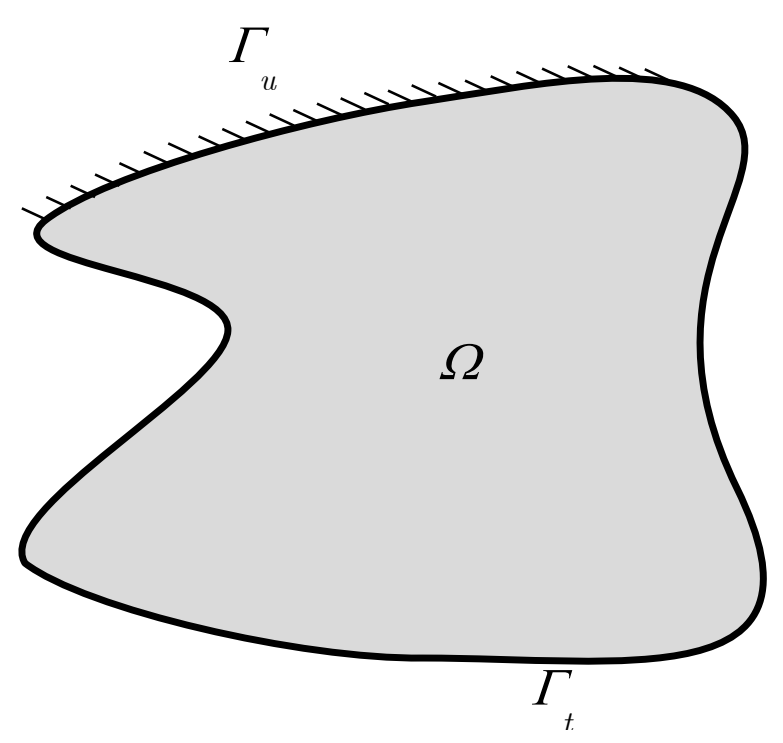

(a)

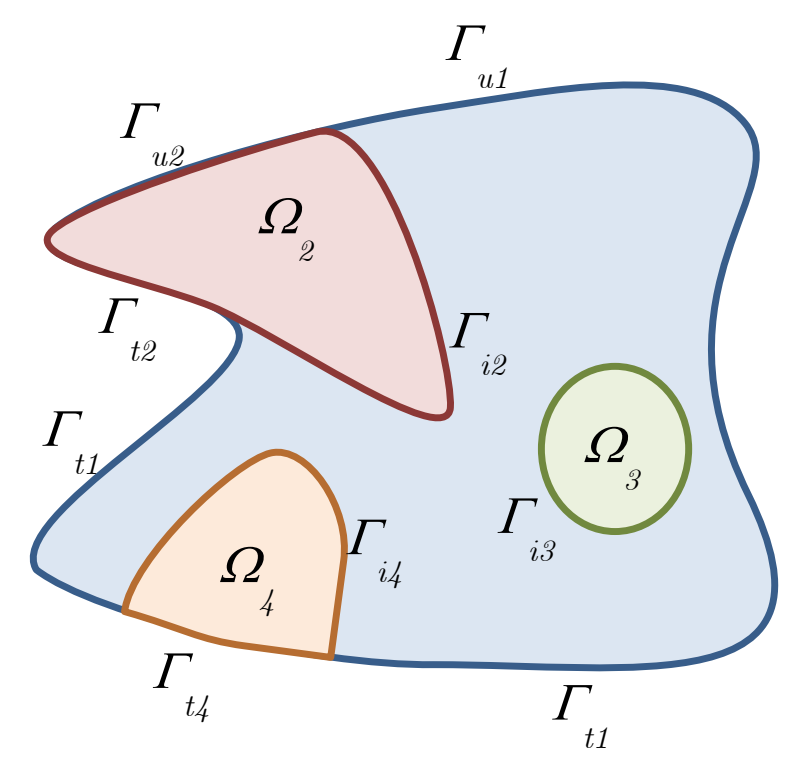

(b)

Figura 9 - Representação do problema em 2D

O deslocamento de cada ponto é expresso como $\boldsymbol{u}(\boldsymbol{x})=u_{1} \boldsymbol{e}_{1}+u_{2} \boldsymbol{e}_{2}$ para o caso bidimensional e $\boldsymbol{u}(\boldsymbol{x})=u_{1} \boldsymbol{e}_{1}+u_{2} \boldsymbol{e}_{2}+u_{3} \boldsymbol{e}_{3}$ em três dimensões. O Gradiente dos Deslocamentos é dado por

$$
L=\frac{\partial \boldsymbol{u}}{\partial \boldsymbol{x}}
$$

e o Tensor das Deformações, para o caso linear,

$$
\boldsymbol{E}=\frac{1}{2}\left(\boldsymbol{L}+\boldsymbol{L}^{T}\right)=\left[\begin{array}{ccc}
\frac{\partial u_{1}}{\partial x_{1}} & \frac{1}{2}\left(\frac{\partial u_{1}}{\partial x_{2}}+\frac{\partial u_{2}}{\partial x_{1}}\right) & \frac{1}{2}\left(\frac{\partial u_{1}}{\partial x_{3}}+\frac{\partial u_{3}}{\partial x_{1}}\right) \\
\frac{1}{2}\left(\frac{\partial u_{1}}{\partial x_{2}}+\frac{\partial u_{2}}{\partial x_{1}}\right) & \frac{\partial u_{2}}{\partial x_{2}} & \frac{1}{2}\left(\frac{\partial u_{2}}{\partial x_{3}}+\frac{\partial u_{3}}{\partial x_{2}}\right) \\
\frac{1}{2}\left(\frac{\partial u_{1}}{\partial x_{3}}+\frac{\partial u_{3}}{\partial x_{1}}\right) & \frac{1}{2}\left(\frac{\partial u_{2}}{\partial x_{3}}+\frac{\partial u_{3}}{\partial x_{2}}\right) & \frac{\partial u_{3}}{\partial x_{3}}
\end{array}\right]
$$

As componentes de $\boldsymbol{E}$ são normalmente agrupadas em um vetor das deformações 


$$
\boldsymbol{\varepsilon}=\left\{\begin{array}{c}
E_{11} \\
E_{22} \\
E_{33} \\
2 E_{12} \\
2 E_{23} \\
2 E_{13}
\end{array}\right\}=\left\{\begin{array}{c}
\varepsilon_{11} \\
\varepsilon_{22} \\
\varepsilon_{33} \\
\gamma_{12} \\
\gamma_{23} \\
\gamma_{13}
\end{array}\right\}=\left\{\begin{array}{c}
\frac{\partial u_{1}}{\partial x_{1}} \\
\frac{\partial u_{2}}{\partial x_{2}} \\
\frac{\partial u_{3}}{\partial x_{3}} \\
\frac{\partial u_{1}}{\partial x_{2}}+\frac{\partial u_{2}}{\partial x_{1}} \\
\frac{\partial u_{2}}{\partial x_{3}}+\frac{\partial u_{3}}{\partial x_{2}} \\
\frac{\partial u_{1}}{\partial x_{3}}+\frac{\partial u_{3}}{\partial x_{1}}
\end{array}\right\},
$$

ou, com a definição do operador diferencial

$$
\boldsymbol{\Delta}=\left[\begin{array}{ccc}
\frac{\partial}{\partial x_{1}} & 0 & 0 \\
0 & \frac{\partial}{\partial x_{2}} & 0 \\
0 & 0 & \frac{\partial}{\partial x_{3}} \\
\frac{\partial}{\partial x_{2}} & \frac{\partial}{\partial x_{1}} & 0 \\
0 & \frac{\partial}{\partial x_{3}} & \frac{\partial}{\partial x_{2}} \\
\frac{\partial}{\partial x_{3}} & 0 & \frac{\partial}{\partial x_{1}}
\end{array}\right]
$$

escreve-se resumidamente

$$
\varepsilon=\Delta u
$$




\subsection{Tensões}

Seja o Tensor das Tensões de Cauchy ${ }^{1}$

$$
\boldsymbol{T}=\left[\begin{array}{lll}
\sigma_{11} & \tau_{12} & \tau_{13} \\
\tau_{12} & \sigma_{22} & \tau_{23} \\
\tau_{13} & \tau_{23} & \sigma_{33}
\end{array}\right]
$$

Suas componentes também podem ser reunidas em um vetor coluna

$$
\boldsymbol{\sigma}=\left\{\begin{array}{llllll}
\sigma_{11} & \sigma_{22} & \sigma_{33} & \tau_{12} & \tau_{23} & \tau_{13}
\end{array}\right\}^{T}
$$

\subsection{Equilíbrio}

O equilíbrio de um elemento infinitesimal provê o equilíbrio local do sólido, válido todos os pontos de cada região $\Omega_{i}$.

$$
\operatorname{div} \boldsymbol{T}+\boldsymbol{b}=\boldsymbol{O}, \text { em } \Omega_{a}, a=1 . . n r e g,
$$

onde $\boldsymbol{b}$ reúne as forças de volume impostas ao corpo. Nas fronteiras naturais, a compatibilidade de tensões é expressa por

$$
\boldsymbol{T} \boldsymbol{n}=\overline{\boldsymbol{t}}, \mathrm{em} \Gamma_{t-a}, a=1 . . n r e g,
$$

onde $\boldsymbol{n}$ é a normal externa a $\Gamma_{t}$. Na fronteira essencial, as condições de contorno de Dirichlet são expressas por

$$
\boldsymbol{u}=\overline{\boldsymbol{u}} \text {, em } \Gamma_{u-a}, a=1 . . n r e g .
$$

\footnotetext{
${ }^{1}$ Definições de tensões fora do regime linear, bem como provas da simetria de $\boldsymbol{T}$ podem ser encontrados em (PIMENTA - APOSTILA)
} 
Descreve-se a continuidade de tensões e de deslocamentos nas fronteira de interface por

$$
\begin{aligned}
\boldsymbol{u}_{\text {mat }} & =\boldsymbol{u}_{\text {agr }}, \\
\boldsymbol{T}_{\text {mat }} \boldsymbol{n} & =\boldsymbol{T}_{a g r} \boldsymbol{n}, \text { em } \Gamma_{i}^{a}, a=2 . . n r e g
\end{aligned}
$$

onde $\boldsymbol{u}_{\text {mat }}$ reúne os deslocamentos dos pontos da matriz em $\Gamma_{i-a}, \boldsymbol{u}_{a g r}$ reúne os deslocamentos dos pontos do agregado em $\Gamma_{i-a}, \boldsymbol{T}_{m a t}$ e $\boldsymbol{T}_{a g r}$ são os Tensores das tensões de Cauchy na matriz e no agregado respectivamente e $\boldsymbol{n}$ é o versor normal à superfície.

(3.11) a (3.14) constituem a forma forte, ou diferencial, do problema.

\subsection{Forma Fraca}

A implementação em Método de Galerkin exige a imposição fraca do equilíbrio nos domínios, o que pode ser obtido através do princípio dos trabalhos virtuais. O trabalho virtual interno é o trabalho virtual realizado pelas tensões quando de um deslocamento virtual $\delta \boldsymbol{u}$ da estrutura. Estes deslocamentos traduzem-se em deformações virtuais $\delta \varepsilon=\Delta \delta \boldsymbol{u}$, de forma que

$$
\delta W_{i n t}=\int_{\Omega} \boldsymbol{\sigma} \cdot \delta \varepsilon d \Omega
$$

O trabalho virtual externo é o trabalho realizado pelas forças externas ao corpo quando deste mesmo deslocamento:

$$
\delta W_{e x t}=\int_{\Omega} \boldsymbol{b} \cdot \delta \boldsymbol{u} d \Omega+\int_{\Gamma_{t}} \boldsymbol{t} \cdot \delta \boldsymbol{u} d \Gamma_{t}+\int_{\Gamma_{u}} \boldsymbol{r} \cdot \delta \boldsymbol{u} d \Gamma_{u},
$$

onde $r$ é a reação de apoio em um ponto da fronteira essencial. É interessante salientar a inclusão do trabalho virtual destas nos trabalhos virtuais externos. Em uma formulação usual em Elementos Finitos, estes são nulos já que é trivial garantir que $\delta \boldsymbol{u}=\boldsymbol{O}$ em $\Gamma_{u}$. A aproximação por Mínimos Quadrados Móveis não tem caráter interpolador, ou seja, as funções de forma não obedecem à propriedade de Delta de Kronecker $\Phi_{i}\left(\boldsymbol{x}_{j}\right)=\delta_{i}$. Pelo 
mesmo motivo, faz-se necessária a inclusão do trabalho virtual complementar das reações de apoio, forma fraca com o que se impõe as condições essenciais de contorno.

$$
\delta W_{\text {rea }}=-\int_{\Gamma_{u}} \delta \boldsymbol{r} \cdot(\boldsymbol{u}-\overline{\boldsymbol{u}}) d \Gamma_{u}=0, \forall \delta \boldsymbol{r}
$$

A imposição fraca do equilíbrio através do Princípio dos Trabalhos Virtuais $\delta W_{i n t}-\delta W_{e x t}=0, \forall \delta \boldsymbol{u}$, junto à imposição fraca das condições essenciais de contorno, gera o funcional representativo do problema global, forma fraca do problema:

$$
\begin{aligned}
& \delta W_{i n t}-\delta W_{\text {ext }}+\delta W_{\text {rea }}=\int_{\Omega} \boldsymbol{\sigma} \cdot \delta \varepsilon d \Omega+ \\
& -\int_{\Omega} \boldsymbol{b} \cdot \delta \boldsymbol{u} d \Omega-\int_{\Gamma_{t}} \boldsymbol{t} \cdot \delta \boldsymbol{u} d \Gamma_{t}-\int_{\Gamma_{u}} \boldsymbol{r} \cdot \delta \boldsymbol{u} d \Gamma_{u}+ \\
& -\int_{\Gamma_{u}} \delta \boldsymbol{r} \cdot(\boldsymbol{u}-\overline{\boldsymbol{u}}) d \Gamma_{u}=0, \forall \delta \boldsymbol{u}, \delta \boldsymbol{r}
\end{aligned}
$$

O equilíbrio deve ser obedecido em todas as regiões do problema. Então, a soma dos funcionais acima correspondentes a todas as regiões também deve ser nula, conforme

$$
\delta W=\sum_{a=1}^{n r e g} \delta W_{a}=0
$$

A compatibilidade de deslocamentos na fronteira de interface também é imposta de forma fraca. Inclui o trabalho virtual das tensões de contato e o trabalho virtual complementar das mesmas, termos advém da variação da potência das tensões na interface. Em cada fronteira, tem-se

$$
\delta W_{\text {interface }}=\int_{\Gamma_{i}} \boldsymbol{\sigma} \cdot\left(\delta \boldsymbol{u}_{\text {mat }}-\delta \boldsymbol{u}_{a g r}\right) d \Gamma_{i}+\int_{\Gamma_{i}} \delta \boldsymbol{\sigma} \cdot\left(\boldsymbol{u}_{m a t}-\boldsymbol{u}_{a g r}\right) d \Gamma_{i} \cdot
$$

A formulação fraca do problema é então representada pelo funcional híbrido de deslocamentos dado por 


$$
\begin{aligned}
\delta W= & \sum_{a=1}^{n r e g} \delta W_{\text {int }}^{a}-\sum_{a=1}^{\text {nreg }} \delta W_{\text {ext }}^{a}+\sum_{a=1}^{\text {nreg }} \delta W_{\text {rea }}^{a}-\sum_{a=2}^{\text {nreg }} \delta W_{\text {int }}^{a} \\
= & \sum_{a=1}^{n r e g} \int_{\Omega^{a}} \boldsymbol{\sigma} \cdot \delta \boldsymbol{\varepsilon} d \Omega^{a}+ \\
& -\sum_{a=1}^{n r e g} \int_{\Omega^{a}} \boldsymbol{b} \cdot \delta \boldsymbol{u} d \Omega^{a}-\sum_{a=1}^{n r e g} \int_{\Gamma_{t}^{a}} \boldsymbol{t} \cdot \delta \boldsymbol{u} d \Gamma_{t}^{a}-\sum_{a=1}^{n r e g} \int_{\Gamma_{t}^{a}} \boldsymbol{r} \cdot \delta \boldsymbol{u} d \Gamma_{t}^{a}+ \\
& -\sum_{a=1}^{n r e g} \int_{\Gamma_{u}^{a}} \delta \boldsymbol{r} \cdot(\boldsymbol{u}-\overline{\boldsymbol{u}}) d \Gamma_{u}^{a}+ \\
& -\sum_{a=2}^{n r e g} \int_{\Gamma_{i}^{a}} \boldsymbol{\sigma}_{i}^{a} \cdot\left(\delta \boldsymbol{u}_{\text {mat }}-\delta \boldsymbol{u}_{\text {agr }}\right) d \Gamma_{i}^{a}-\sum_{a=2}^{n r e g} \int_{\Gamma_{i}^{a}} \delta \boldsymbol{\sigma}_{i}^{a} \cdot\left(\boldsymbol{u}_{\text {mat }}-\boldsymbol{u}_{\text {agr }}\right) d \Gamma_{i}^{a} \\
= & 0, \forall \delta \boldsymbol{u}, \delta \boldsymbol{r}, \delta \boldsymbol{\sigma}_{i}^{a}
\end{aligned}
$$




\section{MÉTODO DE GALERKIN LIVRE DE ELEMENTOS (MGLE)}

Nos últimos anos a tecnologia tem melhorado o desempenho do computador e devido isso a análise numérica tem progredido significativamente. Por isso, hoje é possível simular modelos com elevados números de variáveis.

O método dos elementos finitos (MEF) é amplamente aplicado na análise numérica. Por outro lado, a geração da malha é um processo que precisa de um elevado processamento computacional e tempo de operação. Recentemente, o emprego de métodos sem malha (MSM) tem ganhado espaço em vários campos da Engenharia.

Dentre os métodos sem malha, o MGLE tem sido considerado o mais rápido em processamento e precisão [72] [73] [74] [75] [76]. Esse método se caracteriza pela discretização de um domínio de interesse por um conjunto de nós, colocados arbitrariamente (nuvem de nós), sem que exista explicitamente uma malha estruturada de elementos no sentido convencional, definindo funções. A Figura 10 ilustra a discretização de um RVE, com a diferença básica do MGLE e o MEF para a definição do domínio através da nuvem de partículas e da malha de elementos, respectivamente.

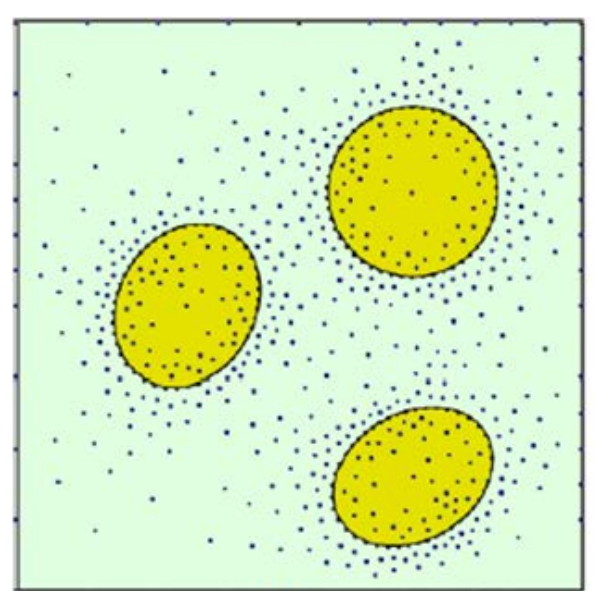

(a)

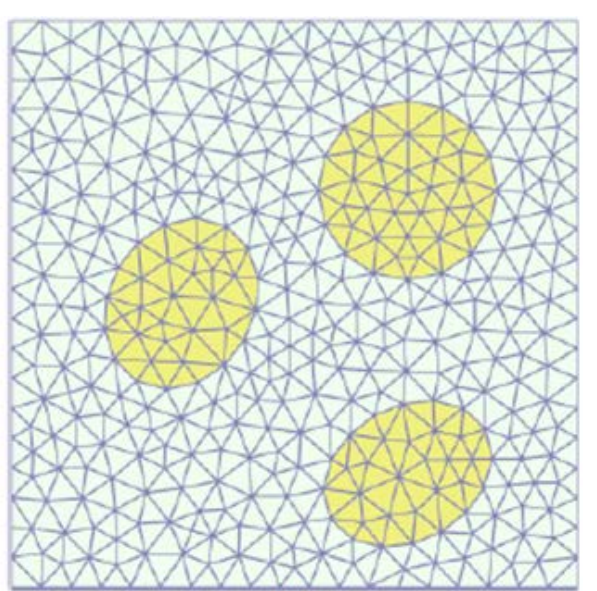

(b)

Figura 10 - (a) Discretização pelo MGLE com nuvem de partículas e (b) Discretização pelo MEF com malha de elementos. 
O MGLE é um método numérico cujas equações básicas de governo do método discreto independem da definição de uma malha de elementos finitos. A solução aproximada do problema, em um espaço de dimensões finitas, é construída sem que a conectividade entre os pontos nodais desta aproximação seja pré-estabelecida. [72] [73] [74] [75] [76] [77] [78] [79] [80] [81]. Os MSM tem tido um desenvolvimento acentuado nos últimos anos, surgindo como uma alternativa ao MEF. Em particular, será utilizada neste estudo, a variação deste método denominada MGLE.

Um caminho adotado para a construção de discretização sem malha é o uso da aproximação por mínimos quadrados moveis (MLS), que é à base de muitos métodos MSM para o ajuste de curvas a partir dos valores da variável de estado associados a um conjunto de nós irregularmente distribuídos no domínio. O método MGLE consiste em usar funções de forma definidas por Mínimos Quadrados Móveis para descrever os campos de aproximação e de teste. [82] [83] [84] [85] [86] [87]

As principais características do MGLE são:

- A construção das funções de base, utilizadas na construção do espaço de aproximação, a partir do método de mínimos quadrados móveis (MLS).

- Ausência da conectividade nodal pré-definida associada ao emprego de elementos.

- A utilização da formulação fraca de Galerkin, na discretização do sistema de equações diferenciais.

- A integração numérica realizada com o emprego de uma estrutura de células ou de uma malha de fundo.

A malha de fundo é uma malha típica de elementos finitos usada apenas durante o procedimento da integração numérica, não contribuindo na definição dos graus de liberdade de discretização, assim, diz-se apenas que a malha de fundo representa o domínio, porém não o discretiza.

A Tabela 1 ilustra a ordem cronológica do surgimento dos principais MSM. 
Tabela 1 - Cronologia dos métodos sem malha [88]

\begin{tabular}{|ccc|}
\hline Métodos & Referencias & $\begin{array}{c}\text { Método de } \\
\text { aproximação }\end{array}$ \\
\hline Método das Partículas & $\begin{array}{c}\text { Lucy 1977, Gingold and } \\
\text { Monaghan 1977, etc. }\end{array}$ & Método de Galerkin \\
\hline Método dos Pontos Finitos & $\begin{array}{c}\text { Liszka and Orkisz 1980, } \\
\text { Onate et al 1996, etc. }\end{array}$ & Diferenças finitas \\
\hline Método do Elemento Difuso & Nayroles et al 1992. & Aproximação por MLS \\
\hline $\begin{array}{c}\text { Método de Galerkin Livre } \\
\text { de Elementos }\end{array}$ & $\begin{array}{c}\text { Belytschko et al 1994, } \\
\text { 1996, 1998, etc. }\end{array}$ & Aproximação por MLS \\
\hline $\begin{array}{c}\text { Método das Funções de } \\
\text { Base Radial }\end{array}$ & $\begin{array}{c}\text { Liu et al 1995, 1996, etc. } \\
\text { Método da Nuvens-hp }\end{array}$ & Método de Galerkin \\
\hline $\begin{array}{c}\text { Método sem malha } \\
\text { Duarte and Oden 1996, } \\
\text { etc. }\end{array}$ & Aproximação por MLS \\
\hline $\begin{array}{c}\text { Método de Petrov-Galerkin } \\
\text { Local }\end{array}$ & $\begin{array}{c}\text { Ytluri and Zhu 1998, } \\
\text { 1996, 1998, etc. }\end{array}$ & Aproximação por MLS \\
& $\begin{array}{c}\text { 1999, Atluri and Shen } \\
\text { 2002, etc. }\end{array}$ & \\
\hline $\begin{array}{c}\text { Método de Interpolação de } \\
\text { Pontos }\end{array}$ & $\begin{array}{c}\text { Liu and Gu 1999, 2001, } \\
\text { Wang and Liu 2000, 2001, } \\
\text { 2002, etc. }\end{array}$ & Interpolação no ponto \\
\hline $\begin{array}{c}\text { Método sem malha forma } \\
\text { forte-fraca }\end{array}$ & $\begin{array}{c}\text { Liu and Gu 2002, 2003, } \\
\text { etc. }\end{array}$ & MLS, PIM, radial PIM \\
\hline
\end{tabular}

As principais vantagens do MGLE estão associadas à simplicidade na definição da discretização e a flexibilidade para modificação desta discretização, dispensando o uso de estratégias sofisticadas de geração de malha. As desvantagens estão relacionadas à necessidade de um número maior de pontos de integração seja suficiente de modo a se garantir uma boa integração, o desenvolvimento de uma estrutura de dados efetiva para redução do custo computacional para a construção das funções de forma e a necessidade do uso de procedimentos especiais, neste caso os multiplicadores de Lagrange, para a imposição das condições de contorno essenciais. [84]

Esta facilidade de refino da discretização relaciona-se muito bem com a aplicação em materiais heterogêneos. Com simples algoritmos de determinação de domínio das partículas, pode-se facilmente melhorar a aproximação na zona de transição da argamassa (região próxima às inclusões). Uma discretização por Elementos Finitos necessita que a compatibilidade entre os elementos seja mantida, tornado custoso o refino de malha. 
Entre as várias funções no MSM, a que reúne boas características para a resolução de problemas estruturais é o MLS. Dentre outras cita-se a sua arbitrária continuidade, a partição da unidade, a coerência, a reprodutibilidade e a flexibilidade em acomodar a aproximação ao domínio. [89] [90] [91] [92] [93] [94]

\subsection{Aproximação por Mínimos Quadrados Móveis}

O MLS é uma técnica que permite definir a aproximação de uma função, a partir de um conjunto de valores conhecidos. Essa função foi introduzida por LANCASTER [95], embora muitos autores considerem este método uma extensão do método de Shepard [96], que foi proposto muito anteriormente.

No MLS os elementos essenciais são uma base de funções polinomiais, funções de ponderações, e uma distribuição de pontos. As constantes que aparecem na função de aproximação são determinadas impondo-se a minimização do erro entre a função de aproximação e os valores nodais. O enriquecimento da aproximação é obtido com monômios de ordem mais alta adicionados à base de funções o que, na maioria das vezes, deve ser acompanhado com a introdução de novos pontos nodais à discretização. [95]

A ordem de consistência de uma aproximação, $C^{k}$, é definida como a ordem arbitrária polinomial que pode ser representada pelo processo de ajuste ou aproximação. Uma das propriedades importantes do MLS é a de que o método é capaz de representar exatamente combinações das funções da base intrínseca $p(x)$, ou seja, a consistência da aproximação depende da ordem monomial utilizada para definir $p(x)$. Se a ordem completa for $k$, a função aproximação gerada terá consistência $C^{k}$. Assim, é possível alcançar uma consistência de ordem $k$ através do uso de

$$
p^{T}(x)=\left[1, x, y, x^{2}, x y, y^{2}, \ldots, x^{k}, \ldots, x y^{k-1}, y^{k}\right]
$$

Para melhor entender o MLS, considera-se o problema de uma função contínua $u(\boldsymbol{x})$ , que deve ser aproximada conhecendo-se apenas seus valores $\boldsymbol{u}_{j}$ em um conjunto de pontos nodais $\boldsymbol{x}_{j}, j=1 . . N$. Na Figura 11 esse problema é ilustrado com o um ajuste de curvas em campo unidimensional. 


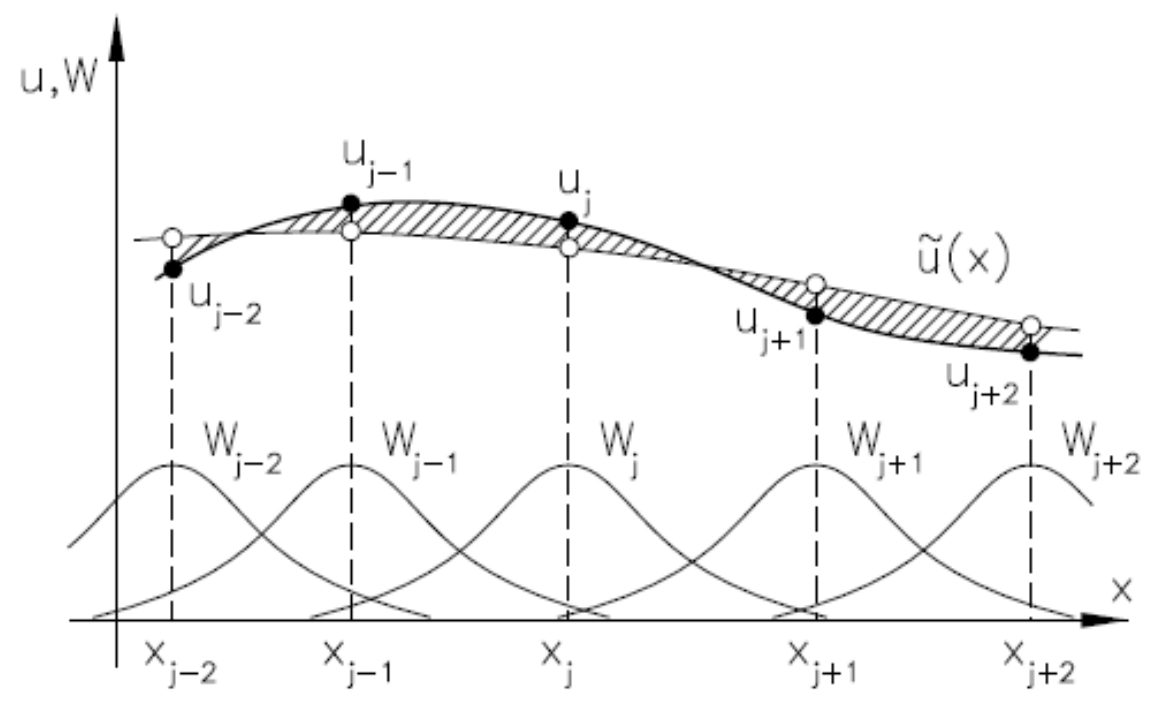

Figura 11 - Método dos Mínimos Quadrados Móveis [119].

Em cada posição de $\boldsymbol{x}$ do domínio, uma aproximação local, $u^{h}(\boldsymbol{x})$, deve ser definida empregando-se um subconjunto de $n(\boldsymbol{x}) \leq N$ pontos vizinhos. Tal aproximação pode ser expressa na forma de uma combinação linear de uma base de funções $\boldsymbol{p}=\left\{p_{i}\right\}_{i=1}^{m},(m \leq n(\boldsymbol{x}))$, segundo os parâmetros $a_{i}$ :

$$
u(x) \cong u^{h}(\boldsymbol{x})=\sum_{i=1}^{m} p_{i}(x) a_{i}(x)=\boldsymbol{p}(\boldsymbol{x})^{T} \boldsymbol{a}(\boldsymbol{x})
$$

\subsection{Função Peso}

Seja, $w_{j}(\boldsymbol{x})$ uma função peso que assuma valores não-nulos apenas na vizinhança do ponto $\boldsymbol{x}_{j}$. Tal função apresenta o suporte compacto, ou seja, $w_{j} \in C_{0}^{l}\left(\boldsymbol{v}_{j}\right)$, onde $l$ representa a continuidade de $w_{j}, \boldsymbol{v}_{j}$ a vizinhança de $\boldsymbol{x}_{j}$ em que a função peso é definida e o zero indica que a função tem valor não-nulo apenas no interior de $\boldsymbol{v}_{j}$. A vizinhança é denominada região de influência do ponto $\boldsymbol{x}_{j}$, sendo limitada por uma medida de referência $r_{j}$ e representada por $\boldsymbol{v}_{j}=\left\{\boldsymbol{x} \in \Omega ;\left\|\boldsymbol{x}-\boldsymbol{x}_{j}\right\| \leq r_{j}\right\}$, conforme a Figura 12. [119] 


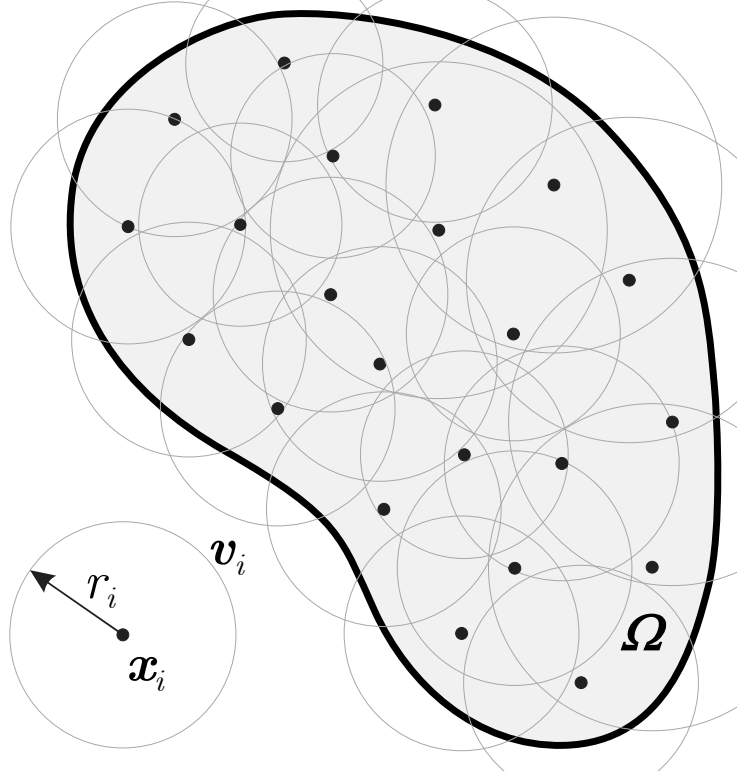

Figura 12 - Representação das nuvens em $\mathbb{R}^{2}$.

Os coeficientes $\boldsymbol{a}(\boldsymbol{x})$ são determinados minimizando-se uma função $J(\boldsymbol{x})$ que reúne as distâncias entre $u^{h}(\boldsymbol{x})$ e $\bar{u}(\boldsymbol{x})$, ponderadas pelas funções $w_{j}$. [119]

$$
J(x)=\sum_{j=1}^{n(x)} w_{j}\left(\boldsymbol{x}-\boldsymbol{x}_{j}\right)\left\{\bar{u}_{j}-u^{h}\left(\boldsymbol{x}_{j}\right)\right\}^{2}
$$

Observa-se que apenas os $n(\boldsymbol{x})$ pontos nodais $\boldsymbol{x}_{j}$ cuja região de influência $\boldsymbol{v}_{j}$ contenha a posição $\boldsymbol{x}$ participam da somatória acima. A relação que determina os coeficientes $\boldsymbol{a}(\boldsymbol{x})$ resulta da minimização de $J(\boldsymbol{x})$ :

$$
\boldsymbol{a}(\boldsymbol{x})=\sum_{j=1}^{n(\boldsymbol{x})} \boldsymbol{A}^{-1}(\boldsymbol{x}) \boldsymbol{B}_{j}(\boldsymbol{x}) \bar{u}_{j}
$$

onde:

$$
\boldsymbol{A}(\boldsymbol{x})=\sum_{r=1}^{n(\boldsymbol{x})} w_{r}\left(\boldsymbol{x}-\boldsymbol{x}_{r}\right) \boldsymbol{p}\left(\boldsymbol{x}_{r}\right) p^{T}\left(\boldsymbol{x}_{r}\right)
$$




$$
\boldsymbol{B}_{j}(\boldsymbol{x})=w_{j}\left(\boldsymbol{x}-\boldsymbol{x}_{j}\right) \boldsymbol{p}\left(\boldsymbol{x}_{j}\right)
$$

Dessa maneira, a aproximação passa a ser escrita como:

$$
u(\boldsymbol{x}) \cong u^{h}(\boldsymbol{x})=\sum_{j=1}^{n(\boldsymbol{x})} \phi_{j}(\boldsymbol{x}) \bar{u}_{j}=\boldsymbol{\Phi}^{T} \overline{\boldsymbol{u}},
$$

sendo $\phi_{j}$ um elemento da base de funções de aproximação, com o mesmo suporte $\boldsymbol{v}_{j}$ das funções peso, dado por:

$$
\phi_{j}(\boldsymbol{x})=\boldsymbol{p}^{T}(\boldsymbol{x}) \boldsymbol{A}^{-1}(\boldsymbol{x}) \boldsymbol{B}_{j}(\boldsymbol{x})
$$

Nota-se que, para a representação vetorial, são definidos os seguintes vetores de parâmetros nodais e de funções de forma, e dado por:

$$
\begin{aligned}
\overline{\boldsymbol{u}}^{T} & =\left[\begin{array}{llll}
\bar{u}_{1} & \bar{u}_{2} & \cdots & \bar{u}_{N}
\end{array}\right] \\
\boldsymbol{\Phi}^{T} & =\left[\begin{array}{llll}
\phi_{1} & \phi_{2} & \cdots & \phi_{N}
\end{array}\right]
\end{aligned}
$$

A existência da inversa de $\boldsymbol{A}(\boldsymbol{x})$ depende da conveniente definição dos $r_{j}$ de modo que se respeite a condição $n(\boldsymbol{x}) \geq m$.

A definição da função peso tem sua importância de modo a se obter uma boa performance do método. Ela deve ser construída de tal forma que seja positiva em seu domínio e apresente um decréscimo em sua magnitude à medida que a distância de $\boldsymbol{x}$ com relação $\boldsymbol{x}_{I}$ aumente. Ou seja, seu valor deve ser grande para um ponto $\boldsymbol{x}_{I}$ próximo de $\boldsymbol{x}$ e ser relativamente pequena para o $\boldsymbol{x}_{I}$ mais distante de $\boldsymbol{x}$. [119]

Serão consideradas apenas funções peso as quais dependam apenas da distância, o raio $r$, entre dois pontos, como segue:

$$
w\left(x-x_{I}\right)=w_{I}(r)=w\left(r_{I}\right)
$$


em que

$$
r_{I}=\left\|x-x_{I}\right\|
$$

Assim, a escolha adequada das funções peso é arbitrária à medida que a função seja positiva e contínua, juntamente com suas derivadas até o grau desejado. A escolha do tamanho do suporte da função peso deve garantir que a matriz momento $\boldsymbol{A}(\boldsymbol{x})$ definida seja invertível.

De acordo com as referências de LIU et al [97] e BEISSEL \& BELYTSCHKO [82] a distribuição de partículas deve satisfazer uma condição de estabilidade para que exista a inversa de $\boldsymbol{A}(\boldsymbol{x})$. Neste estudo usará a seguinte função peso spline cúbica:

$$
w(r)=\left\{\begin{array}{l}
\frac{2}{3}-4 r^{2}+4 r^{3}, \text { para } \mathrm{r} \leq \frac{1}{2} \\
\frac{4}{3}-4 r+4 r^{2}-\frac{4}{3} r^{3}, \text { para } \frac{1}{2} \leq \mathrm{r} \leq 1 \\
0, \text { para } \mathrm{r}>1
\end{array}\right.
$$

\subsection{Forma Fraca Discretizada}

Os deslocamentos em cada região são aproximados com funções de forma de Mínimos Quadrados Móveis. O mesmo é feito para o campo de deslocamentos virtuais, ou campo de teste, em uma projeção de Galerkin. É criado um conjunto de $N^{a}$ partículas para cada região do problema, de forma que a aproximação dos deslocamentos nos pontos pertencentes àquela região é dada por

$$
\begin{aligned}
& \boldsymbol{u}(\boldsymbol{x})=\Phi^{a}(\boldsymbol{x}) \boldsymbol{d}^{a} \\
& \left\{\begin{array}{l}
u_{1}(\boldsymbol{x}) \\
u_{2}(\boldsymbol{x}) \\
u_{3}(\boldsymbol{x})
\end{array}\right\}=\left[\begin{array}{cccccccccc}
\Phi_{1} & 0 & 0 & \Phi_{2} & 0 & 0 & \cdots & \Phi_{N^{a}} & 0 & 0 \\
0 & \Phi_{1} & 0 & 0 & \Phi_{2} & 0 & \cdots & 0 & \Phi_{N^{a}} & 0 \\
0 & 0 & \Phi_{1} & 0 & 0 & \Phi_{2} & \cdots & 0 & 0 & \Phi_{N^{a}}
\end{array}\right]\left[\begin{array}{c}
d_{1} \\
d_{2} \\
d_{3} \\
\vdots \\
d_{3 N^{a}}
\end{array}\right], \boldsymbol{x} \in \Omega^{a} .
\end{aligned}
$$


Em (4.14), $\Phi_{i}$ é a função de forma atrelada à partícula $i$ avaliada em $\boldsymbol{x}$. A Figura 13 representa as partículas usadas para a aproximação em Mínimos Quadrados Móveis em três dimensões para um RVE contendo um agregado esférico. É interessante notar a concentração de partículas próximas à interface, região onde os gradientes são mais importantes, e que não há restrições quanto ao uso de partículas fora do domínio que representam.

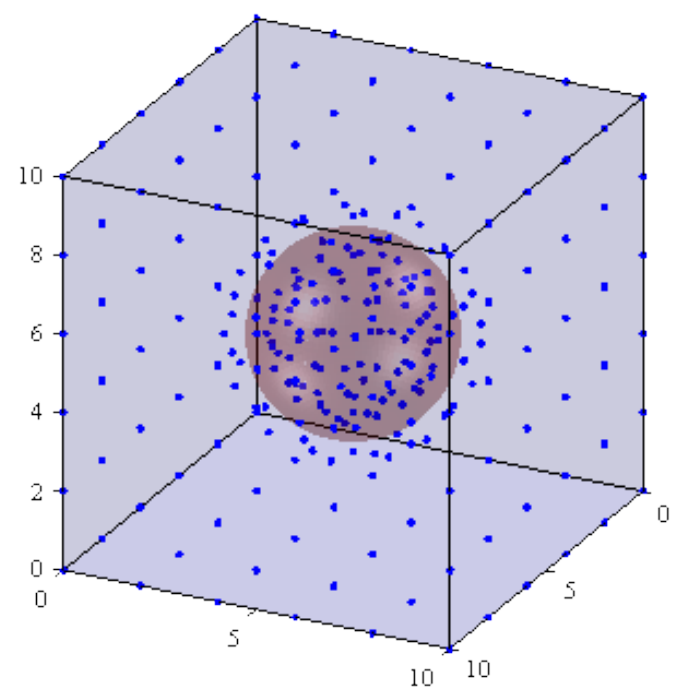

(a)

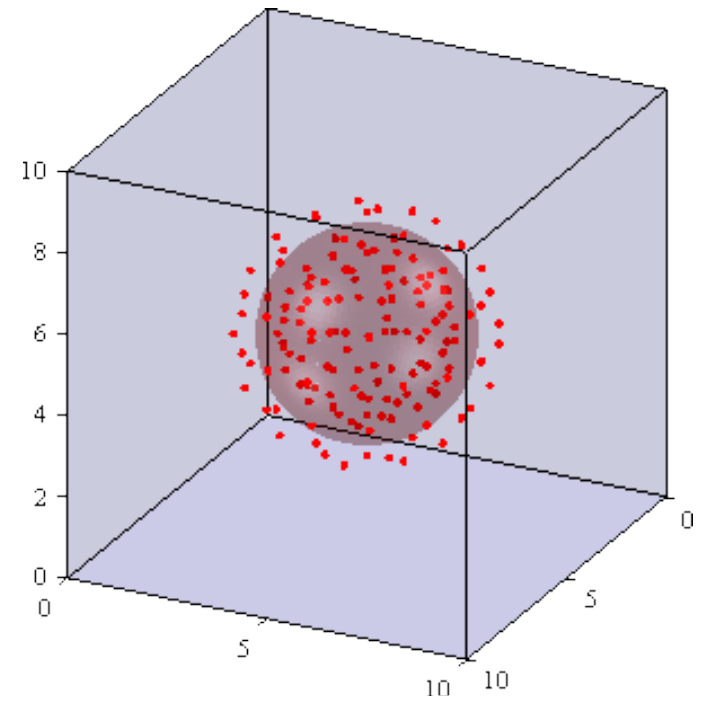

(b)

Figura 13 - Distribuição de partículas (a) na matriz (b) no agregado.

Há, também a necessidade de aproximarem-se as reações de apoio na fronteira essencial, isso é feito utilizando-se funções de forma de elementos finitos bi-dimensionais nos casos 3D e unidimensionais para os casos no plano. A Figura 14 esquematiza essa discretização para o caso do cubo. Sendo $N_{u^{a}}$ o número de nós da fronteira essencial pertencentes à região $a$, a aproximação será dada por 


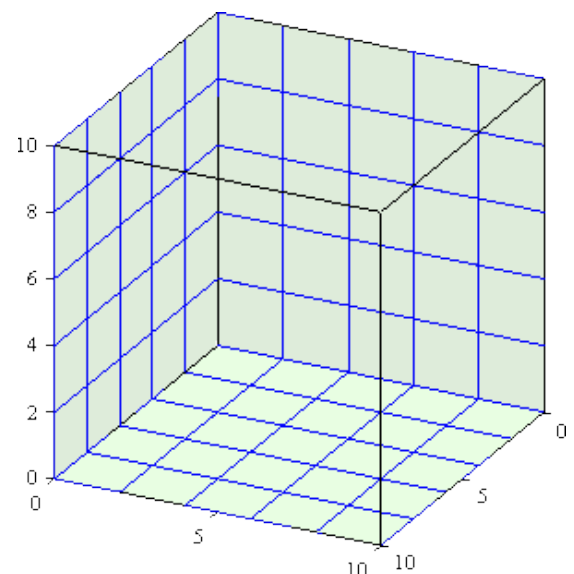

Figura 14 - Discretização da Fronteira Essencial em Elementos Finitos.

$$
\begin{aligned}
& \boldsymbol{r}(\boldsymbol{x})=\boldsymbol{N}^{a}(\boldsymbol{x}) \boldsymbol{\lambda}^{a} \\
& \left\{\begin{array}{l}
r_{1}(\boldsymbol{x}) \\
r_{2}(\boldsymbol{x}) \\
r_{3}(\boldsymbol{x})
\end{array}\right\}=\left[\begin{array}{cccccccccc}
N_{1} & 0 & 0 & N_{2} & 0 & 0 & \cdots & N_{N u^{a}} & 0 & 0 \\
0 & N_{1} & 0 & 0 & N_{2} & 0 & \cdots & 0 & N_{N u^{a}} & 0 \\
0 & 0 & N_{1} & 0 & 0 & N_{2} & \cdots & 0 & 0 & N_{N u^{a}}
\end{array}\right]\left[\begin{array}{c}
\lambda_{1}^{a} \\
\lambda_{2}^{a} \\
\lambda_{3}^{a} \\
\vdots \\
\lambda_{3 N u^{a}}^{a}
\end{array}\right], \boldsymbol{x} \in \Gamma_{u}^{a} \cdot{ }^{\cdot}
\end{aligned}
$$

As tensões de contato nas interfaces também devem ser aproximadas. Aqui, uma nova abordagem foi experimentada, nos casos tridimensionais, onde estas tensões foram aproximadas por Mínimos Quadrados Móveis em um espaço bidimensional paramétrico e mapeadas ao contorno do agregado, tido como um elipsoide de revolução. A Figura 15 mostra essa distribuição no espaço paramétrico $\phi, \theta$ e em $\mathbb{R}^{3}$. Essa aproximação pode ser representada por

$$
\begin{aligned}
& \boldsymbol{\sigma}_{i}^{a}(\boldsymbol{x})=\left\{\begin{array}{c}
\sigma_{i x}^{a} \\
\sigma_{i y}^{a}
\end{array}\right\}=\boldsymbol{\Psi}^{a}(\boldsymbol{x}) \boldsymbol{\lambda}_{i}^{a} \\
& \boldsymbol{\Psi}^{a}=\left[\begin{array}{ccccccc}
\Psi_{1}^{a} & 0 & \Psi_{2}^{a} & 0 & \cdots & \Psi_{N i^{a}}^{a} & 0 \\
0 & \Psi_{1}^{a} & 0 & \Psi_{2}^{a} & \cdots & 0 & \Psi_{N i^{a}}^{a}
\end{array}\right]\left[\begin{array}{c}
\lambda_{i 1}^{a} \\
\lambda_{i 2}^{a} \\
\lambda_{i 3}^{a} \\
\vdots \\
\lambda_{i 3 N i^{a}}^{a}
\end{array}\right], \boldsymbol{x} \in \Gamma_{i}^{a}, a=2 . . n r e g
\end{aligned}
$$

onde $N i^{a}$ é o número de pontos na superfície $\Gamma_{i}^{a}$ utilizados para fazer esta aproximação. 
(a)

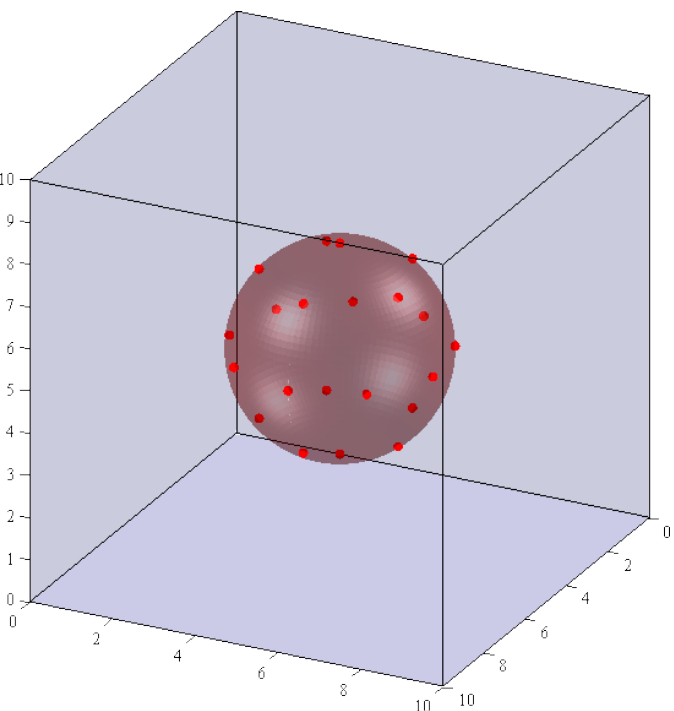

(b)

Figura 15 - Distribuição de partículas na interface. (a) no espaço paramétrico (b) no espaço tridimensional.

As variações dos campos acima aproximados são tomadas com as mesmas aproximações que os campos, ou seja

$$
\begin{aligned}
\delta \boldsymbol{u}^{a}(\boldsymbol{x}) & =\boldsymbol{\Phi}^{a}(\boldsymbol{x}) \delta \boldsymbol{d}^{a}, \boldsymbol{x} \in \Omega^{a} \\
\delta \boldsymbol{r}^{a}(\boldsymbol{x}) & =\boldsymbol{N}^{a}(\boldsymbol{x}) \delta \boldsymbol{\lambda}^{a}, \boldsymbol{x} \in \Gamma_{u}^{a} \\
\delta \boldsymbol{\sigma}_{i}^{a}(\boldsymbol{x}) & =\boldsymbol{\Psi}^{a}(\boldsymbol{x}) \delta \boldsymbol{\lambda}_{i}^{a}, \boldsymbol{x} \in \Gamma_{i}^{a} \\
\delta \boldsymbol{\varepsilon}(\boldsymbol{x}) & =\boldsymbol{\Delta} \delta \boldsymbol{u}(\boldsymbol{x})=\boldsymbol{\Delta} \boldsymbol{\Phi}^{a}(\boldsymbol{x}) \delta \boldsymbol{d}^{a}=\boldsymbol{B}(\boldsymbol{x}) \delta \boldsymbol{d}^{a}, \boldsymbol{x} \in \Omega^{a} \\
\boldsymbol{\Delta} \boldsymbol{\Phi}^{a}(\boldsymbol{x}) & =\boldsymbol{B}(\boldsymbol{x})
\end{aligned}
$$

Desta forma, o potencial (3.21) pode ser aproximado como 


$$
\begin{aligned}
\delta W= & \sum_{a=1}^{n r e g} \int_{\Omega^{a}} \boldsymbol{\sigma} \cdot\left(\boldsymbol{B}(\boldsymbol{x}) \delta \boldsymbol{d}^{a}\right) d \Omega^{a}+ \\
& -\sum_{a=1}^{n r e g} \int_{\Omega^{a}} \boldsymbol{b} \cdot\left(\boldsymbol{\Phi}^{a}(\boldsymbol{x}) \delta \boldsymbol{d}^{a}\right) d \Omega^{a}-\sum_{a=1}^{n r e g} \int_{\Gamma_{t}^{a}} \boldsymbol{t} \cdot\left(\boldsymbol{\Phi}^{a}(\boldsymbol{x}) \delta \boldsymbol{d}^{a}\right) d \Gamma_{t}^{a}+ \\
& -\sum_{a=1}^{n r e g} \int_{\Gamma_{t}^{a}}\left(\boldsymbol{N}^{a}(\boldsymbol{x}) \boldsymbol{\lambda}^{a}\right) \cdot\left(\boldsymbol{\Phi}^{a}(\boldsymbol{x}) \delta \boldsymbol{d}^{a}\right) d \Gamma_{t}^{a}+ \\
& -\sum_{a=1}^{n r e g} \int_{\Gamma_{u}^{a}}\left(\boldsymbol{N}^{a}(\boldsymbol{x}) \delta \boldsymbol{\lambda}^{a}\right) \cdot\left(\boldsymbol{\Phi}^{a}(\boldsymbol{x}) \boldsymbol{d}^{a}-\overline{\boldsymbol{u}}\right) d \Gamma_{u}^{a}+ \\
& -\sum_{a=2}^{n r e g} \int_{\Gamma_{i}^{a}}\left(\boldsymbol{\Psi}^{a}(\boldsymbol{x}) \boldsymbol{\lambda}_{i}^{a}\right) \cdot\left(\boldsymbol{\Phi}^{\text {mat }}(\boldsymbol{x}) \delta \boldsymbol{d}^{\text {mat }}-\boldsymbol{\Phi}^{a}(\boldsymbol{x}) \delta \boldsymbol{d}^{a}\right) d \Gamma_{i}^{a}+ \\
& -\sum_{a=2}^{n r e g} \int_{\Gamma_{i}^{a}}\left(\boldsymbol{\Psi}^{a}(\boldsymbol{x}) \delta \boldsymbol{\lambda}_{i}^{a}\right) \cdot\left(\boldsymbol{\Phi}^{\text {mat }}(\boldsymbol{x}) \boldsymbol{d}^{\text {mat }}-\boldsymbol{\Phi}^{a}(\boldsymbol{x}) \boldsymbol{d}^{a}\right) d \Gamma_{i}^{a} \\
= & 0, \forall \delta \boldsymbol{d}^{a}, \delta \boldsymbol{\lambda}^{a}, \delta \boldsymbol{\lambda}_{i}^{a}
\end{aligned}
$$

que em forma matricial gera

$$
\begin{aligned}
\delta W= & \sum_{a=1}^{n r e g} \delta \boldsymbol{d}^{a T}\left[\int_{\Omega^{a}} \boldsymbol{B}^{T} \boldsymbol{\sigma} d \Omega^{a}\right]+ \\
& -\sum_{a=1}^{n r e g} \delta \boldsymbol{d}^{a T}\left[\int_{\Omega^{a}} \boldsymbol{\Phi}^{a T} \boldsymbol{b} d \Omega^{a}\right]-\sum_{a=1}^{n r e g} \delta \boldsymbol{d}^{a T}\left[\int_{\Gamma_{t}^{a}} \boldsymbol{\Phi}^{a T} \boldsymbol{t} d \Gamma_{t}^{a}\right]+ \\
& -\sum_{a=1}^{n r e g} \delta \boldsymbol{d}^{a T}\left[\int_{\Gamma_{t}^{a}} \boldsymbol{\Phi}^{a T} \boldsymbol{N}^{a} d \Gamma_{t}^{a}\right] \boldsymbol{\lambda}^{a}+ \\
& -\sum_{a=1}^{n r e g} \delta \boldsymbol{\lambda}^{a T}\left[\int_{\Gamma_{u}^{a}} \boldsymbol{N}^{a T} \boldsymbol{\Phi}^{a} d \Gamma_{u}^{a}\right] \boldsymbol{d}^{a}+\sum_{a=1}^{n r e g} \delta \boldsymbol{\lambda}^{a T}\left[\int_{\Gamma_{u}^{a}} \boldsymbol{N}^{a T} \overline{\boldsymbol{u}} d \Gamma_{u}^{a}\right]+ \\
& -\sum_{a=2}^{n r e g} \delta \boldsymbol{d}^{m a t T}\left[\int_{\Gamma_{i}^{a}} \boldsymbol{\Phi}^{m a t T} \boldsymbol{\Psi}^{a} d \Gamma_{i}^{a}\right] \boldsymbol{\lambda}_{i}^{a}+\sum_{a=2}^{n r e g} \delta \boldsymbol{d}^{a T}\left[\int_{\Gamma_{i}^{a}} \boldsymbol{\Phi}^{a T} \boldsymbol{\Psi}^{a} d \Gamma_{i}^{a}\right] \boldsymbol{\lambda}_{i}^{a}+ \\
& -\sum_{a=2}^{n r e g} \delta \boldsymbol{\lambda}_{i}^{a T}\left[\int_{\Gamma_{i}^{a}} \boldsymbol{\Psi}^{a T} \boldsymbol{\Phi}^{m a t} d \Gamma_{i}^{a}\right] \boldsymbol{d}^{\text {mat }}+\sum_{a=2}^{n r e g} \delta \boldsymbol{\lambda}_{i}^{a T}\left[\int_{\Gamma_{i}^{a}} \boldsymbol{\Psi}^{a T} \boldsymbol{\Phi}^{a} d \Gamma_{i}^{a}\right] \boldsymbol{d}^{a} \\
= & 0, \forall \delta \boldsymbol{d}^{a}, \delta \boldsymbol{\lambda}^{a}, \delta \boldsymbol{\lambda}_{i}^{a}
\end{aligned}
$$

onde foram exploradas as independências de $\boldsymbol{d}, \boldsymbol{\lambda}, \boldsymbol{\lambda}_{i}$ e suas respectivas variações no domínio e foram omitidas as dependências das matrizes em relação à posição. Fazendo-se, em turnos, cada elemento de $\delta \boldsymbol{d}^{a}, \delta \boldsymbol{\lambda}^{a}, \delta \boldsymbol{\lambda}_{i}^{a}$ nulo, obtém-se as equações vetoriais:

para a matriz, $a=1$ : 


$$
\begin{aligned}
& \boldsymbol{r}^{m a t}=\underbrace{\int_{\Omega^{m a t}} \boldsymbol{B}^{T} \boldsymbol{\sigma} d \Omega^{m a t}}_{\boldsymbol{f}_{\text {int }}^{\text {mat }}\left(\boldsymbol{d}^{\text {mat }}\right)}-[\underbrace{\left[\int_{\Omega^{m a t}} \boldsymbol{\Phi}^{\text {mat } T} \boldsymbol{b} d \Omega^{m a t}+\int_{\Gamma_{t}^{m a t}} \boldsymbol{\Phi}^{\text {mat } T} \boldsymbol{t} d \Gamma_{t}^{\text {mat }}\right]}_{\boldsymbol{f}_{\text {ext }}^{\text {mat }}}+
\end{aligned}
$$

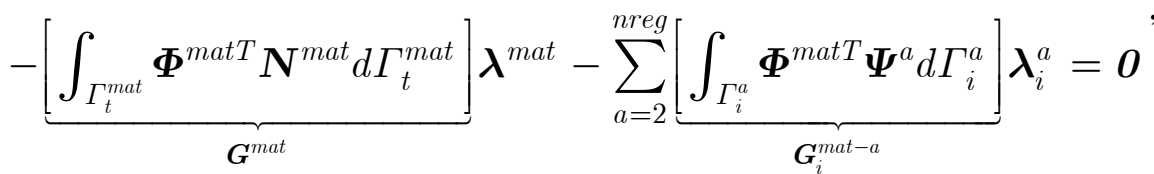

onde foi explicitada a dependência de $f_{\text {int }}^{\text {mat }}$ com relação aos parâmetros nodais $\boldsymbol{d}^{\text {mat }}$, além de definir-se

$$
\begin{aligned}
& \boldsymbol{f}_{\text {int }}^{\text {mat }}\left(\boldsymbol{d}^{m a t}\right)=\int_{\Omega^{m a t}} \boldsymbol{B}^{T} \boldsymbol{\sigma}\left(\boldsymbol{d}^{m a t}\right) d \Omega^{m a t} \\
& \boldsymbol{f}_{\text {ext }}^{\text {mat }}=\int_{\Omega^{m a t}} \boldsymbol{\Phi}^{m a t T} \boldsymbol{b} d \Omega^{m a t}+\int_{\Gamma_{t}^{m a t}} \boldsymbol{\Phi}^{m a t T} \boldsymbol{t} d \Gamma_{t}^{\text {mat }} \\
& \boldsymbol{G}^{\text {mat }}=\int_{\Gamma_{t}^{\text {mat }}} \boldsymbol{\Phi}^{m a t T} \boldsymbol{N}^{m a t} d \Gamma_{t}^{m a t} \\
& \boldsymbol{G}_{i}^{\text {mat }-a}=\int_{\Gamma_{i}^{a}} \boldsymbol{\Phi}^{m a t T} \boldsymbol{\Psi}^{a} d \Gamma_{i}^{a}
\end{aligned}
$$

Desta forma, pode-se exprimir o resíduo na matriz como

$$
\boldsymbol{r}^{\text {mat }}\left(\boldsymbol{d}^{\text {mat }}\right)=\boldsymbol{f}_{\text {int }}^{\text {mat }}\left(\boldsymbol{d}^{\text {mat }}\right)-\boldsymbol{f}_{\text {ext }}^{\text {mat }}-\boldsymbol{G}^{m a t} \boldsymbol{\lambda}^{\text {mat }}-\sum_{a=2}^{\text {nreg }} \boldsymbol{G}_{i}^{\text {mat-a}} \boldsymbol{\lambda}_{i}^{a}=\boldsymbol{O} .
$$

Também pode-se extrair de (4.19) os resíduos referentes a cada agregado

$$
\begin{aligned}
& \boldsymbol{r}^{a g r}=\underbrace{\int_{\Omega^{a}} \boldsymbol{B}^{T} \boldsymbol{\sigma}\left(\boldsymbol{d}^{a}\right) d \Omega^{a}}_{\boldsymbol{f}_{\text {int }}^{a g r-a}\left(\boldsymbol{d}^{a}\right)}-[\underbrace{\left[\int_{\Omega^{a}} \boldsymbol{\Phi}^{a T} \boldsymbol{b} d \Omega^{a}+\int_{\Gamma_{t}^{a}} \boldsymbol{\Phi}^{a T} \boldsymbol{t} d \Gamma_{t}^{a}\right]}_{\boldsymbol{f}_{e x t}^{a g r-a}}+ \\
& -\underbrace{\int_{\Gamma_{t}^{a}} \boldsymbol{\Phi}^{a T} \boldsymbol{N}^{a} d \Gamma_{t}^{a}}_{\boldsymbol{G}^{a g r-a}} \boldsymbol{\lambda}^{a}+\underbrace{\int_{\Gamma_{i}^{a}} \boldsymbol{\Phi}^{a T} \boldsymbol{\Psi}^{a} d \Gamma_{i}^{a} \boldsymbol{\lambda}_{i}^{a}=\boldsymbol{O}, i=2 . . n r e g}_{\boldsymbol{G}_{i}^{\text {agr }-a}}
\end{aligned}
$$

Com as definições de 


$$
\begin{aligned}
\boldsymbol{f}_{i n t}^{a g r-a}\left(\boldsymbol{d}^{a}\right) & =\int_{\Omega^{a}} \boldsymbol{B}^{T} \boldsymbol{\sigma}\left(\boldsymbol{d}^{a}\right) d \Omega^{a} \\
\boldsymbol{f}_{e x t}^{a g r-a} & =\int_{\Omega^{a}}^{\boldsymbol{\Phi}^{a T}} \boldsymbol{b} d \Omega^{a}+\int_{\Gamma_{t}^{a}} \boldsymbol{\Phi}^{a T} \boldsymbol{t} d \Gamma_{t}^{a} \\
\boldsymbol{G}^{a g r-a} & =\int_{\Gamma_{t}^{a}} \boldsymbol{\Phi}^{a T} \boldsymbol{N}^{a} d \Gamma_{t}^{a} \\
\boldsymbol{G}_{i}^{a g r-a} & =\int_{\Gamma_{i}^{a}}^{\boldsymbol{\Phi}^{a T}} \boldsymbol{\Psi}^{a} d \Gamma_{i}^{a}
\end{aligned}
$$

(4.23) pode ser reescrito na forma

$$
\boldsymbol{r}^{a g r}\left(\boldsymbol{d}^{a}\right)=\boldsymbol{f}_{i n t}^{a g r-a}\left(\boldsymbol{d}^{a}\right)-\boldsymbol{f}_{e x t}^{a g r-a}-\boldsymbol{G}^{a g r-a} \boldsymbol{\lambda}^{a}+\boldsymbol{G}_{i}^{a g r-a} \boldsymbol{\lambda}_{i}^{a}=\boldsymbol{O} .
$$

Da mesma forma, pode-se escrever, a partir de (4.19)

$$
\boldsymbol{r}^{u-a}=-\underbrace{\int_{\Gamma_{u}^{a}} \boldsymbol{N}^{a T} \boldsymbol{\Phi}^{a} d \Gamma_{u}^{a}}_{\boldsymbol{G}^{a T}} \boldsymbol{d}^{a}+\underbrace{\int_{\Gamma_{u}^{a}} \boldsymbol{N}^{a T} \overline{\boldsymbol{u}} d \Gamma_{u}^{a}}_{\boldsymbol{q}^{a}}=\boldsymbol{O}
$$

e definir-se

$$
\boldsymbol{q}^{a}=\int_{\Gamma_{u}^{a}} \boldsymbol{N}^{a T} \overline{\boldsymbol{u}} d \Gamma_{u}^{a}
$$

para cada região $a$. Sendo $\boldsymbol{G}^{a}$ definido em (4.21) para a matriz e em (4.24) para o agregado, (4.26) pode ser escrito como

$$
\boldsymbol{r}^{u-a}=-\boldsymbol{G}^{a T} \boldsymbol{d}^{a}+\boldsymbol{q}^{a}=\boldsymbol{O}
$$

Por fim, o vetor resíduo na fronteira de interface pode ser lido

$$
\boldsymbol{r}^{i n t-a}=\underbrace{\int_{\Gamma_{i}^{a}} \boldsymbol{\Psi}^{a T} \boldsymbol{\Phi}^{m a t} d \Gamma_{i}^{a} \boldsymbol{d}^{m a t}}_{\boldsymbol{G}_{i}^{\text {mat }-a T}}+\underbrace{\int_{\Gamma_{i}^{a}} \boldsymbol{\Psi}^{a T} \boldsymbol{\Phi}^{a} d \Gamma_{i}^{a}}_{\boldsymbol{G}_{i}^{a g r-a T}} \boldsymbol{d}^{a}=\boldsymbol{O},
$$

com $G_{i}^{\text {mat }-a}$ e $G_{i}^{a g r-a}$ definidas em (4.21) e (4.23) respectivamente,

$$
\boldsymbol{r}^{i n t-a}=\boldsymbol{G}_{i}^{m a t-a T} \boldsymbol{d}^{m a t}+\boldsymbol{G}_{i}^{a g r-a T} \boldsymbol{d}^{a}=\boldsymbol{O} .
$$


Os vetores de resíduo podem ser reunidos em um vetor único

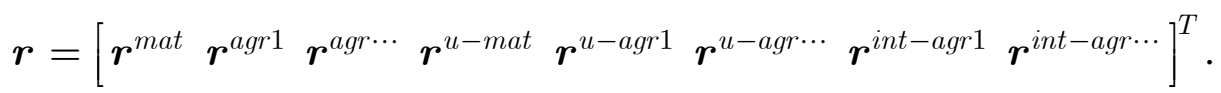

\subsection{Problemas Elásticos}

Para fim de validação da formulação e de estabelecer-se o tamanho necessário ao RVE, alguns problemas foram resolvidos sem considerar-se a danificação do material. Para tal, uma relação linear entre as tensões e as deformações foi admitida em todas as regiões do problema e em todos os níveis de carga. Além disso, o material foi considerado elástico e isótropo. Desta forma, pode-se escrever

$$
\sigma=D \varepsilon
$$

Essa relação, comumente chamada neo-hookeana, pode ser descrita com apenas duas variáveis intrínsecas ao material. Uma forma tradicional de fazê-lo é através das constantes de Lamé $\lambda$ (módulo de deformabilidade volumétrica) e $\mu$ (módulo de deformabilidade por cisalhamento).

$$
\boldsymbol{T}=2 \mu \boldsymbol{E}+\lambda \operatorname{tr}(\boldsymbol{E}) \boldsymbol{I}
$$

Outras duas grandezas comumente utilizadas são o módulo de Young $E$ e o módulo de Poisson $\nu$, que se relacionam com $\mu$ e $\lambda$ por (2.8) e (2.9).

Conforme descrito em (3.6) e (3.10), as componentes de $\boldsymbol{T}$ e de $\boldsymbol{E}$ podem ser rearranjadas em $\sigma$ e $\varepsilon$. A partir de (4.33) pode-se, então, obter 


$$
\boldsymbol{D}=\frac{E}{(1+\nu)(1-2 \nu)}\left[\begin{array}{cccccc}
1-\nu & \nu & \nu & 0 & 0 & 0 \\
\nu & 1-\nu & \nu & 0 & 0 & 0 \\
\nu & \nu & 1-\nu & 0 & 0 & 0 \\
0 & 0 & 0 & \frac{(1-2 \nu)}{2} & 0 & 0 \\
0 & 0 & 0 & 0 & \frac{(1-2 \nu)}{2} & 0 \\
0 & 0 & 0 & 0 & 0 & \frac{(1-2 \nu)}{2}
\end{array}\right]
$$

Para os problemas planos, foi admitido o Estado Plano de Tensão, onde os vetores de tensão e de deformação são respectivamente

$$
\begin{aligned}
& \boldsymbol{\sigma}=\left[\begin{array}{lll}
\sigma_{11} & \sigma_{22} & \tau_{12}
\end{array}\right]^{T} \\
& \boldsymbol{\varepsilon}=\left[\begin{array}{lll}
\varepsilon_{11} & \varepsilon_{22} & \gamma_{12}
\end{array}\right]^{T}
\end{aligned}
$$

Em tal situação, a matriz de rigidez é simplificada para

$$
\boldsymbol{D}=\frac{E}{\left(1-\nu^{2}\right)}\left[\begin{array}{ccc}
1 & \nu & 0 \\
\nu & 1-\nu & 0 \\
0 & 0 & \frac{(1-\nu)}{2}
\end{array}\right]
$$

Nesses casos, o vetor de forças internas definidos em (4.21) e (4.24) será expresso por

$$
\begin{aligned}
& \boldsymbol{f}_{i n t}^{a}=\int_{\Omega^{a}} \boldsymbol{B}^{T} \boldsymbol{\sigma} d \Omega^{a}=\int_{\Omega^{a}} \boldsymbol{B}^{T} \boldsymbol{D} \boldsymbol{\varepsilon} d \Omega^{a} \\
& =\int_{\Omega^{a}} \boldsymbol{B}^{T} \boldsymbol{D} \boldsymbol{B} \boldsymbol{d}^{a} d \Omega^{a}=\underbrace{\int_{\Omega^{a}} \boldsymbol{B}^{T} \boldsymbol{D} \boldsymbol{B} d \Omega^{a}}_{\boldsymbol{K}^{a}} \boldsymbol{d}^{a} .
\end{aligned}
$$

Ou seja, surge a matriz de rigidez da região

$$
\boldsymbol{K}^{a}=\int_{\Omega^{a}} \boldsymbol{B}^{T} \boldsymbol{D} \boldsymbol{B} d \Omega^{a}
$$


de forma que as forças internas serão dadas por

$$
f_{i n t}^{a}=\boldsymbol{K}^{a} \boldsymbol{d}^{a} .
$$

O resíduo interno, dado por (4.22) e (4.26) será, então

$$
\boldsymbol{r}^{a}=\boldsymbol{K}^{a} \boldsymbol{d}^{a}-\boldsymbol{f}_{\text {ext }}^{a}-\boldsymbol{G}^{a} \boldsymbol{\lambda}^{a}+\boldsymbol{G}_{i}^{a} \boldsymbol{\lambda}_{i}^{a}=\boldsymbol{O},
$$

e o resíduo geral será

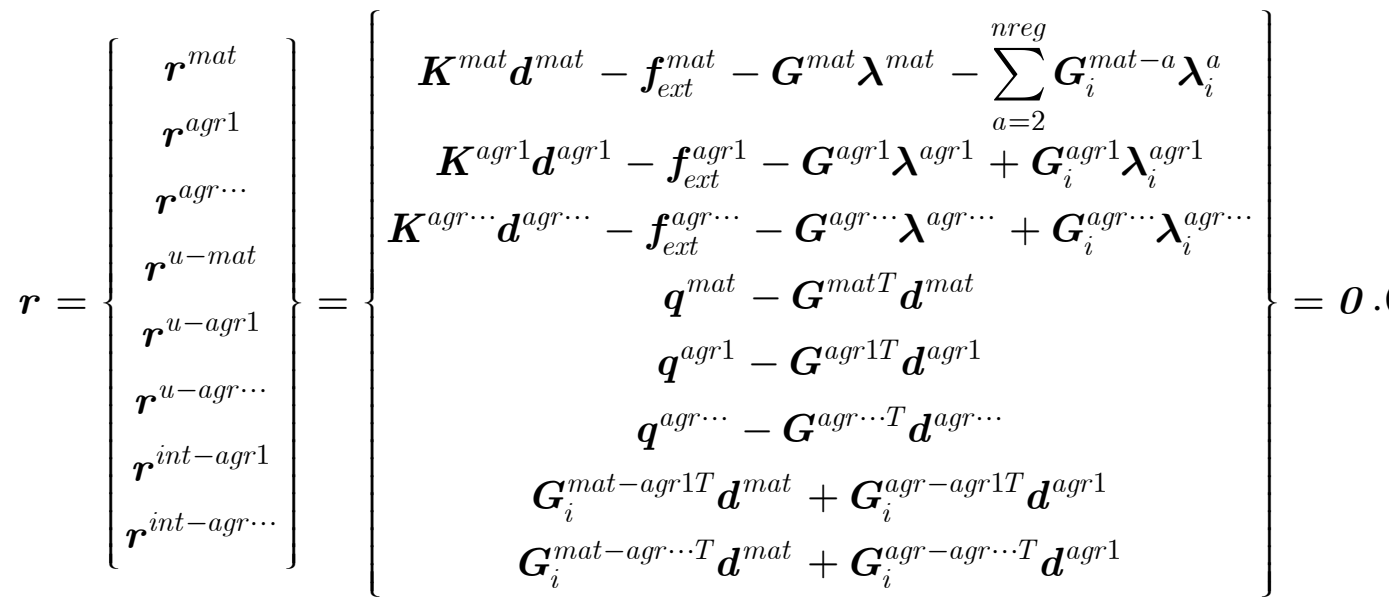

A equação (4.41) é um sistema linear de equações em $\boldsymbol{d}^{\text {mat }}, \boldsymbol{d}^{\text {agr1 }}, \boldsymbol{d}^{\text {agr } \cdots}, \boldsymbol{\lambda}^{\text {mat }}, \boldsymbol{\lambda}^{\text {agr1 }}$ , $\boldsymbol{\lambda}^{a g r \cdots}, \boldsymbol{\lambda}_{i}^{a g r 1}$ e $\boldsymbol{\lambda}_{i}^{a g r \cdots}$, que pode ser expresso por

$$
\left[\begin{array}{ccc}
\boldsymbol{K} & \boldsymbol{G} & \boldsymbol{G}_{i} \\
\boldsymbol{G}^{T} & \boldsymbol{O} & \boldsymbol{O} \\
\boldsymbol{G}_{i}^{T} & \boldsymbol{O} & \boldsymbol{O}
\end{array}\right]\left\{\begin{array}{c}
\boldsymbol{d} \\
\boldsymbol{\lambda} \\
\boldsymbol{\lambda}_{i}
\end{array}\right\}=\left\{\begin{array}{c}
\boldsymbol{f}_{e x t} \\
\boldsymbol{q} \\
\boldsymbol{o}
\end{array}\right\},
$$

onde

$$
\boldsymbol{K}=\left[\begin{array}{ccc}
\boldsymbol{K}^{\text {mat }} & \boldsymbol{O} & \boldsymbol{O} \\
\boldsymbol{O} & \boldsymbol{K}^{a g r 1} & \boldsymbol{O} \\
\boldsymbol{O} & \boldsymbol{O} & \boldsymbol{K}^{a g r \cdots}
\end{array}\right]
$$




$$
\begin{aligned}
& \boldsymbol{G}=\left[\begin{array}{ccc}
-\boldsymbol{G}^{\text {mat }} & \boldsymbol{O} & \boldsymbol{O} \\
\boldsymbol{O} & -\boldsymbol{G}^{a g r 1} & \boldsymbol{O} \\
\boldsymbol{O} & \boldsymbol{O} & -\boldsymbol{G}^{a g r \cdots}
\end{array}\right], \\
& \boldsymbol{G}_{i}=\left[\begin{array}{cc}
\boldsymbol{G}_{i}^{\text {mat-agr } 1} & \boldsymbol{G}_{i}^{\text {mat-agr } \cdots} \\
\boldsymbol{G}_{i}^{a g r-a g r 1} & \boldsymbol{O} \\
\boldsymbol{O} & \boldsymbol{G}_{i}^{a g r-a g r \cdots}
\end{array}\right], \\
& \boldsymbol{d}=\left\{\begin{array}{lll}
\boldsymbol{d}^{m a t} & \boldsymbol{d}^{a g r 1} \boldsymbol{d}^{a g r \cdots}
\end{array}\right\}^{T}, \\
& \boldsymbol{q}=\left\{-\boldsymbol{q}^{m a t}-\boldsymbol{q}^{a g r 1}-\boldsymbol{q}^{a g r \cdots}\right\}^{T} \mathrm{e} \\
& \boldsymbol{f}^{e x t}=\left\{\boldsymbol{f}_{\text {ext }}^{\text {mat }} \boldsymbol{f}_{\text {ext }}^{\text {agr } 1} \boldsymbol{f}_{\text {ext }}^{\text {agr } \cdots}\right\}^{T} .
\end{aligned}
$$

\subsection{Problemas com Dano}

Em problemas que envolvam a evolução do dano com o nível de deformações, a relação constitutiva deixa de ser linear e (4.39) deixa de ser válido. O problema deixa de ser linear, constituindo um sistema de equações não linear em $\boldsymbol{d}$. Supondo que o dano só ocorra na matriz e que os agregados mantêm-se no regime linear, essa não-linearidade se dará em $\boldsymbol{d}^{\text {mat }}$ apenas.

Faz-se necessário um esquema de solução de equações não lineares. A escolha recai sobre o processo iterativo de Newton-Raphson. A partir de um valor inicial para a solução

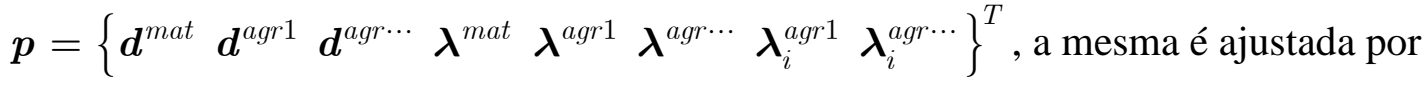

$$
\boldsymbol{p}_{k+1}=\boldsymbol{p}_{k}-\boldsymbol{\delta}
$$

onde 


$$
\begin{aligned}
& \boldsymbol{M}_{k} \boldsymbol{\delta}=\boldsymbol{r}\left(\boldsymbol{p}_{k}\right) \\
& \boldsymbol{M}_{k}=\left[\frac{\partial \boldsymbol{r}}{\partial \boldsymbol{p}}\right]_{k} .
\end{aligned}
$$

A matriz $\boldsymbol{M}$ é dita matriz de rigidez tangente do problema, e pode ser calculada por

$$
\begin{aligned}
& \boldsymbol{M}=\left[\begin{array}{ccc}
\frac{\partial \boldsymbol{r}^{a}}{\partial \boldsymbol{d}^{a}} & \frac{\partial \boldsymbol{r}^{a}}{\partial \boldsymbol{\lambda}^{a}} & \frac{\partial \boldsymbol{r}^{a}}{\partial \boldsymbol{\lambda}_{i}^{a}} \\
\frac{\partial \boldsymbol{r}^{u-a}}{\partial \boldsymbol{d}^{a}} & \frac{\partial \boldsymbol{r}^{u-a}}{\partial \boldsymbol{\lambda}^{a}} & \frac{\partial \boldsymbol{r}^{u-a}}{\partial \boldsymbol{\lambda}_{i}^{a}} \\
\frac{\partial \boldsymbol{r}^{i n t-a}}{\partial \boldsymbol{d}^{a}} & \frac{\partial \boldsymbol{r}^{i n t-a}}{\partial \boldsymbol{\lambda}^{a}} & \frac{\partial \boldsymbol{r}^{i n t-a}}{\partial \boldsymbol{\lambda}_{i}^{a}}
\end{array}\right] \text {, onde } \\
& \frac{\partial \boldsymbol{r}^{a}}{\partial \boldsymbol{d}^{a}}=\left[\begin{array}{lll}
\frac{\partial \boldsymbol{r}^{m a t}}{\partial \boldsymbol{d}^{\text {mat }}} & \frac{\partial \boldsymbol{r}^{m a t}}{\partial \boldsymbol{d}^{a g r 1}} & \frac{\partial \boldsymbol{r}^{m a t}}{\partial \boldsymbol{d}^{a g r \cdots}} \\
\frac{\partial \boldsymbol{r}^{a g r 1}}{\partial \boldsymbol{d}^{\text {mat }}} & \frac{\partial \boldsymbol{r}^{a g r 1}}{\partial \boldsymbol{d}^{a g r 1}} & \frac{\partial \boldsymbol{r}^{a g r 1}}{\partial \boldsymbol{d}^{a g r \cdots}} \\
\frac{\partial \boldsymbol{r}^{a g r \cdots}}{\partial \boldsymbol{d}^{\text {mat }}} & \frac{\partial \boldsymbol{r}^{a g r \cdots}}{\partial \boldsymbol{d}^{a g r 1}} & \frac{\partial \boldsymbol{r}^{a g r \cdots}}{\partial \boldsymbol{d}^{a g r \cdots}}
\end{array}\right]=\left[\begin{array}{ccc}
\boldsymbol{K}^{m a t} & \boldsymbol{O} & \boldsymbol{O} \\
\boldsymbol{O} & \boldsymbol{K}^{a g r 1} & \boldsymbol{O} \\
\boldsymbol{0} & \boldsymbol{O} & \boldsymbol{K}^{a g r \cdots}
\end{array}\right]
\end{aligned}
$$

sendo $\boldsymbol{K}^{a}$ uma versão de (4.38) onde a relação constitutiva $\boldsymbol{D}$ depende do estado de deformação do material,

$$
\frac{\partial \boldsymbol{r}^{a}}{\partial \boldsymbol{\lambda}^{a}}=\left[\begin{array}{lll}
\frac{\partial \boldsymbol{r}^{m a t}}{\partial \boldsymbol{\lambda}^{m a t}} & \frac{\partial \boldsymbol{r}^{m a t}}{\partial \boldsymbol{\lambda}^{a g r 1}} & \frac{\partial \boldsymbol{r}^{m a t}}{\partial \boldsymbol{\lambda}^{a g r \cdots}} \\
\frac{\partial \boldsymbol{r}^{a g r 1}}{\partial \boldsymbol{\lambda}^{m a t}} & \frac{\partial \boldsymbol{r}^{a g r 1}}{\partial \boldsymbol{\lambda}^{a g r 1}} & \frac{\partial \boldsymbol{r}^{a g r 1}}{\partial \boldsymbol{\lambda}^{a g r \cdots}} \\
\frac{\partial \boldsymbol{r}^{a g r \cdots}}{\partial \boldsymbol{\lambda}^{\text {mat }}} & \frac{\partial \boldsymbol{r}^{a g r \cdots}}{\partial \boldsymbol{\lambda}^{a g r 1}} & \frac{\partial \boldsymbol{r}^{a g r \cdots}}{\partial \boldsymbol{\lambda}^{a g r \cdots}}
\end{array}\right]=\left[\begin{array}{ccc}
-\boldsymbol{G}^{m a t} & \boldsymbol{O} & \boldsymbol{O} \\
\boldsymbol{O} & -\boldsymbol{G}^{a g r 1} & \boldsymbol{O} \\
\boldsymbol{O} & \boldsymbol{O} & -\boldsymbol{G}^{a g r \cdots}
\end{array}\right]=\boldsymbol{G}
$$




$$
\begin{aligned}
& \frac{\partial \boldsymbol{r}^{a}}{\partial \boldsymbol{\lambda}_{i}^{a}}=\left[\begin{array}{ll}
\frac{\partial \boldsymbol{r}^{m a t}}{\partial \boldsymbol{\lambda}_{i}^{a g r 1}} & \frac{\partial \boldsymbol{r}^{m a t}}{\partial \boldsymbol{\lambda}_{i}^{a g r \cdots}} \\
\frac{\partial \boldsymbol{r}^{a g r 1}}{\partial \boldsymbol{\lambda}_{i}^{a g r 1}} & \frac{\partial \boldsymbol{r}^{a g r 1}}{\partial \boldsymbol{\lambda}_{i}^{a g r \cdots}} \\
\frac{\partial \boldsymbol{r}^{a g r \cdots}}{\partial \boldsymbol{\lambda}_{i}^{a g r 1}} & \frac{\partial \boldsymbol{r}^{a g r \cdots}}{\partial \boldsymbol{\lambda}_{i}^{a g r \cdots}}
\end{array}\right]=\left[\begin{array}{cc}
\boldsymbol{G}_{i}^{\text {mat-agr } 1} & \boldsymbol{G}_{i}^{\text {mat-agr } \cdots} \\
\boldsymbol{G}_{i}^{a g r-a g r 1} & \boldsymbol{O} \\
\boldsymbol{O} & \boldsymbol{G}_{i}^{a g r-a g r \cdots}
\end{array}\right]=\boldsymbol{G}_{i} \\
& \begin{aligned}
\frac{\partial \boldsymbol{r}^{u-a}}{\partial \boldsymbol{d}^{a}} & =\left[\begin{array}{ccc}
\frac{\partial \boldsymbol{r}^{u-m a t}}{\partial \boldsymbol{d}^{\text {mat }}} & \frac{\partial \boldsymbol{r}^{u-m a t}}{\partial \boldsymbol{d}^{a g r 1}} & \frac{\partial \boldsymbol{r}^{u-m a t}}{\partial \boldsymbol{d}^{a g r \cdots}} \\
\frac{\partial \boldsymbol{r}^{u-a g r 1}}{\partial \boldsymbol{d}^{\text {mat }}} & \frac{\partial \boldsymbol{r}^{u-a g r 1}}{\partial \boldsymbol{d}^{a g r 1}} & \frac{\partial \boldsymbol{r}^{u-a g r 1}}{\partial \boldsymbol{d}^{a g r \cdots}} \\
\frac{\partial \boldsymbol{r}^{u-a g r \cdots}}{\partial \boldsymbol{d}^{\text {mat }}} & \frac{\partial \boldsymbol{r}^{u-a g r \cdots}}{\partial \boldsymbol{d}^{a g r 1}} & \frac{\partial \boldsymbol{r}^{u-a g r \cdots}}{\partial \boldsymbol{d}^{a g r \cdots}}
\end{array}\right] \\
& =\left[\begin{array}{ccc}
-\boldsymbol{G}^{\text {matT }} & \boldsymbol{O} & \boldsymbol{O} \\
\boldsymbol{O} & -\boldsymbol{G}^{a g r 1 T} & \boldsymbol{O} \\
\boldsymbol{O} & \boldsymbol{O} & -\boldsymbol{G}^{a g r \cdots T}
\end{array}\right]=\boldsymbol{G}^{T}
\end{aligned} \\
& \frac{\partial \boldsymbol{r}^{u-a}}{\partial \boldsymbol{\lambda}^{a}}=\left[\begin{array}{ccc}
\frac{\partial \boldsymbol{r}^{u-m a t}}{\partial \boldsymbol{\lambda}^{m a t}} & \frac{\partial \boldsymbol{r}^{u-m a t}}{\partial \boldsymbol{\lambda}^{a g r 1}} & \frac{\partial \boldsymbol{r}^{u-m a t}}{\partial \boldsymbol{\lambda}^{a g r \cdots}} \\
\frac{\partial \boldsymbol{r}^{u-a g r 1}}{\partial \boldsymbol{\lambda}^{m a t}} & \frac{\partial \boldsymbol{r}^{u-a g r 1}}{\partial \boldsymbol{\lambda}^{a g r 1}} & \frac{\partial \boldsymbol{r}^{u-a g r 1}}{\partial \boldsymbol{\lambda}^{a g r \cdots}} \\
\frac{\partial \boldsymbol{r}^{u-a g r \cdots}}{\partial \boldsymbol{\lambda}^{\text {mat }}} & \frac{\partial \boldsymbol{r}^{u-a g r \cdots}}{\partial \boldsymbol{\lambda}^{a g r 1}} & \frac{\partial \boldsymbol{r}^{u-a g r \cdots}}{\partial \boldsymbol{\lambda}^{a g r \cdots}}
\end{array}\right]=\boldsymbol{O} \\
& \frac{\partial \boldsymbol{r}^{u-a}}{\partial \boldsymbol{\lambda}_{i}^{a}}=\left[\begin{array}{cc}
\frac{\partial \boldsymbol{r}^{u-m a t}}{\partial \boldsymbol{\lambda}_{i}^{a g r 1}} & \frac{\partial \boldsymbol{r}^{u-m a t}}{\partial \boldsymbol{\lambda}_{i}^{a g r \cdots}} \\
\frac{\partial \boldsymbol{r}^{u-a g r 1}}{\partial \boldsymbol{\lambda}_{i}^{a g r 1}} & \frac{\partial \boldsymbol{r}^{u-a g r 1}}{\partial \boldsymbol{\lambda}_{i}^{a g r \cdots}} \\
\frac{\partial \boldsymbol{r}^{u-a g r \cdots}}{\partial \boldsymbol{\lambda}_{i}^{a g r 1}} & \frac{\partial \boldsymbol{r}^{u-a g r \cdots}}{\partial \boldsymbol{\lambda}_{i}^{a g r \cdots}}
\end{array}\right]=\boldsymbol{O}
\end{aligned}
$$




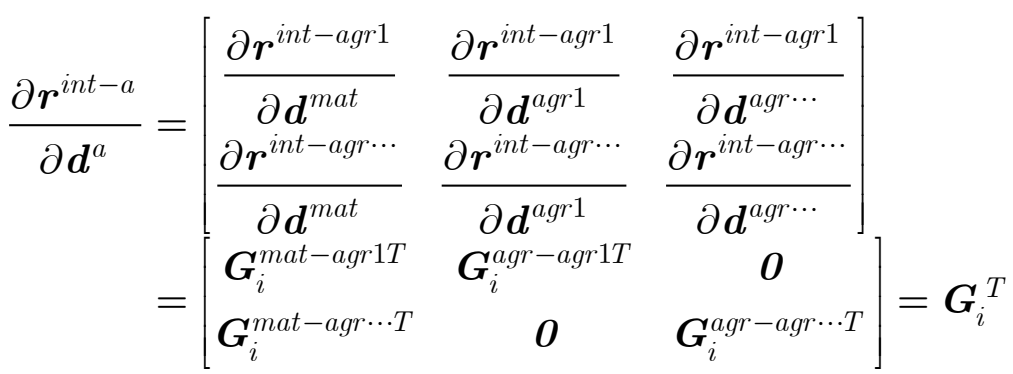

$$
\begin{aligned}
& \frac{\partial \boldsymbol{r}^{i n t-a}}{\partial \boldsymbol{\lambda}^{a}}=\left[\begin{array}{ccc}
\frac{\partial \boldsymbol{r}^{i n t-a g r 1}}{\partial \boldsymbol{\lambda}^{\text {mat }}} & \frac{\partial \boldsymbol{r}^{i n t-a g r 1}}{\partial \boldsymbol{\lambda}^{a g r 1}} & \frac{\partial \boldsymbol{r}^{i n t-a g r 1}}{\partial \boldsymbol{\lambda}^{a g r \cdots}} \\
\frac{\partial \boldsymbol{r}^{i n t-a g r \cdots}}{\partial \boldsymbol{\lambda}^{\text {mat }}} & \frac{\partial \boldsymbol{r}^{i n t-a g r \cdots}}{\partial \boldsymbol{\lambda}^{\text {agr } 1}} & \frac{\partial \boldsymbol{r}^{i n t-a g r \cdots}}{\partial \boldsymbol{\lambda}^{a g r \cdots}}
\end{array}\right]=\boldsymbol{O} \\
& \frac{\partial \boldsymbol{r}^{i n t-a}}{\partial \boldsymbol{\lambda}_{i}^{a}}=\left[\begin{array}{cc}
\frac{\partial \boldsymbol{r}^{i n t-a g r 1}}{\partial \boldsymbol{\lambda}_{i}^{a g r 1}} & \frac{\partial \boldsymbol{r}^{i n t-a g r 1}}{\partial \boldsymbol{\lambda}_{i}^{a g r \cdots}} \\
\frac{\partial \boldsymbol{r}^{i n t-a g r \cdots}}{\partial \boldsymbol{\lambda}_{i}^{a g r 1}} & \frac{\partial \boldsymbol{r}^{i n t-a g r \cdots}}{\partial \boldsymbol{\lambda}_{i}^{a g r \cdots}}
\end{array}\right]=\boldsymbol{O},
\end{aligned}
$$

ou seja,

$$
\boldsymbol{M}=\left[\begin{array}{ccc}
\boldsymbol{K}\left(\boldsymbol{d}^{m a t}\right) & \boldsymbol{G} & \boldsymbol{G}_{i} \\
\boldsymbol{G}^{T} & \boldsymbol{O} & \boldsymbol{O} \\
\boldsymbol{G}_{i}^{T} & \boldsymbol{O} & \boldsymbol{O}
\end{array}\right]
$$

Para o modelo de dano de Mazars, utilizado neste trabalho, a obtenção de $\boldsymbol{D}=\partial \boldsymbol{\sigma} / \partial \boldsymbol{\varepsilon}$ pode ser complicada. Duas aproximações foram experimentadas e serão comparadas adiante: utilizar a matriz obtida na origem, ainda no regime elástico; e penalizar a contribuição dos pontos onde há dano para a matriz de rigidez $\boldsymbol{K}$ de um fator igual a $(1-d)$ , onde $d$ é o dano no local. No primeiro, a matriz $\boldsymbol{M}$ é constante por todo o problema e computada apenas na primeira iteração. Na segunda, $M$ é montada em cada iteração. 
Neste capítulo é apresentada a metodologia de desenvolvimento dos modelos computacionais bidimensionais da heterogeneidade do concreto, admitindo a presença das duas fases características: argamassa e agregado graúdo. Para o agregado foi admitida uma aproximação das formas geométricas para circunferências e elipses (Figura 16) e as propriedades adotadas foram as da brita basáltica. As Tabela 2 e 3 descrevem as propriedades e parâmetros dos materiais utilizados como dados de entrada nos modelos.

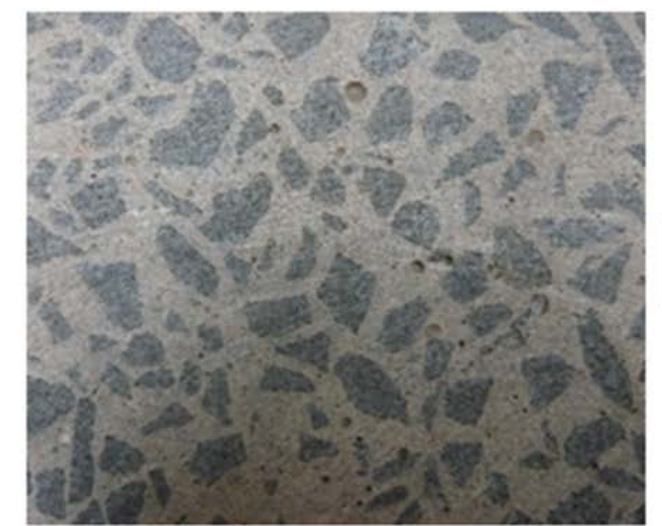

(a)

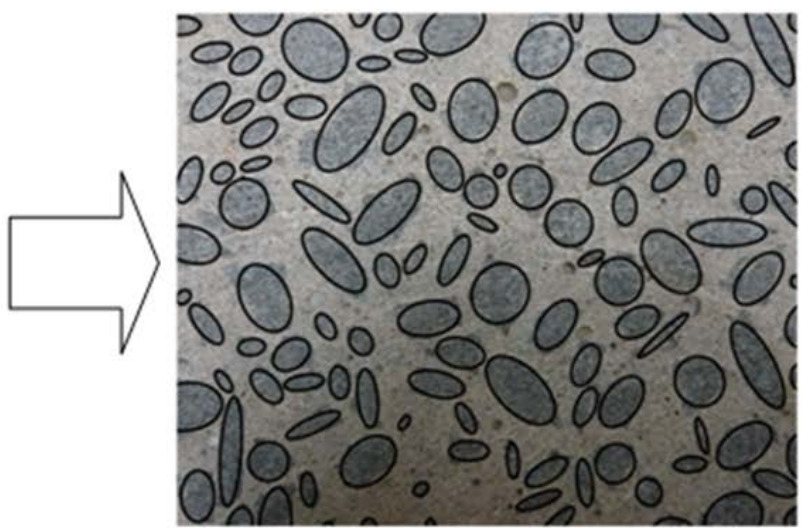

(b)

Figura 16 - (a) Concreto (b) Aproximação para círculos e elipses da geometria dos agregados graúdos.

Tabela 2 - Propriedades dos componentes Módulo de Elasticidade ( $E, M P a$ ) Coeficiente de Poisson $(\nu)$

\begin{tabular}{|ccc|}
\hline Argamassa & $30 \times 10^{3}$ & 0,30 \\
\hline Agregado Graúdo & $50 \times 10^{4}$ & 0,13 \\
\hline
\end{tabular}

Tabela 3 - Parâmetros do modelo constitutivo de Mazars

\begin{tabular}{|cc|}
\hline \multicolumn{2}{|c|}{ Parâmetros do Modelo de Mazars } \\
\hline $\mathrm{A}_{\mathrm{T}}=0,995$ & $\mathrm{~A}_{\mathrm{C}}=0,85$ \\
\hline $\mathrm{B}_{\mathrm{T}}=8000$ & $\mathrm{~B}_{\mathrm{C}}=1050$ \\
\hline$\varepsilon_{\mathrm{d} 0}=0,00007$ & \\
\hline
\end{tabular}

Como ambiente de programação e solver do sistema linear foi utilizado a plataforma Matlab. Outra aproximação admitida foi o fato de não considerar nos modelos a presença da zona de transição entre o agregado graúdo e a argamassa e a presença de vazios espalhados no domínio do material. Essas simplificações foram necessárias devido a precisarem de um 
processamento computacional elevado, logo sendo um limitante para o maior detalhamento dos modelos. Para os exemplos desenvolvidos foi admitido que o material é isotrópico e devido isso bastou um experimento numérico. No caso deste estudo optou-se pelo de compressão axial com deslocamentos prescritos em uma fronteira essencial e restringida na outra fronteira (Figura 17).

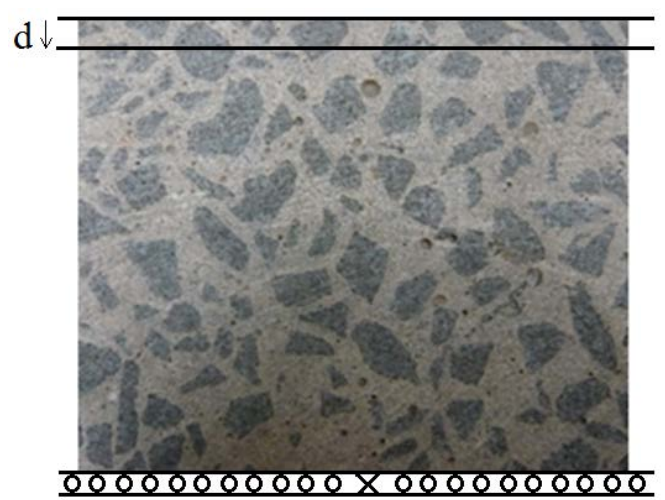

Figura 17 - Esquema do ensaio de compressão axial.

Na integração numérica é necessário criar uma célula de integração para efeitos de integração de forma para obtenção das equações que regem o fenômeno. Nesta pesquisa utilizou-se uma malha de integração de pontos de quadratura de Gauss baseada na nuvem de partículas.

\subsection{Fluxograma Do Algoritmo}

O fluxograma apresentado neste item corresponde ao caso mais geral desenvolvido dentre os modelos analisados. Correspondendo para o caso com a não linearidade da matriz, argamassa, através do conceito de dano contínuo. O fluxograma ilustra o método incrementaliterativo de Newton-Raphson. Para os casos lineares o fluxograma dos modelos finaliza na solução do sistema linear e consequente obtenção dos deslocamentos, tensões e deformações. O procedimento nos modelos bi - e tri - dimensionais foram os mesmos. 


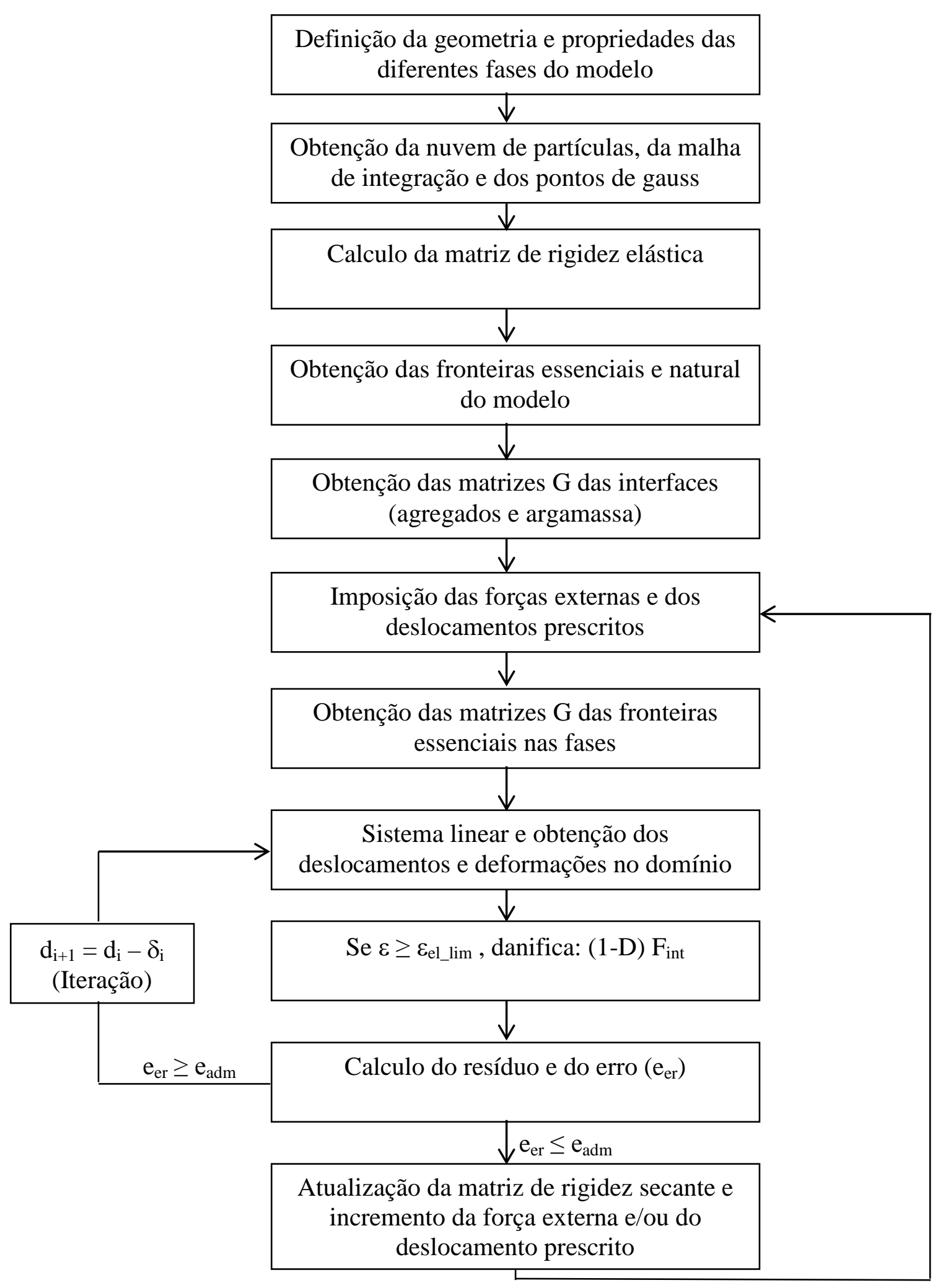




\subsection{Modelo De Material Heterogêneo Com Uma Inclusão}

Inicialmente foram desenvolvidos modelos com apenas uma inclusão (agregado graúdo) e nas diferentes localizações possíveis:

- Inclusão circular no interior da geometria

- Inclusão elíptica no interior da geometria

- Inclusão elíptica na fronteira essencial

- Inclusão elíptica na fronteira natural

As inclusões elípticas foram consideradas com ângulos de inclinação aleatórios. Dessa forma, podem-se representar os diferentes casos de inclusões pretendidos nas simulações numéricas da mecânica do concreto.

Nesses modelos, a geometria adotada foi de quadrados de $10 \mathrm{~cm} \mathrm{x} 10 \mathrm{~cm}$. O sólido foi submetido a uma força de compressão no limite superior e no limite inferior foram restringidos os deslocamentos. A figura 15 ilustra os deslocamentos da vertical quando submetido a um deslocamento prescrito.

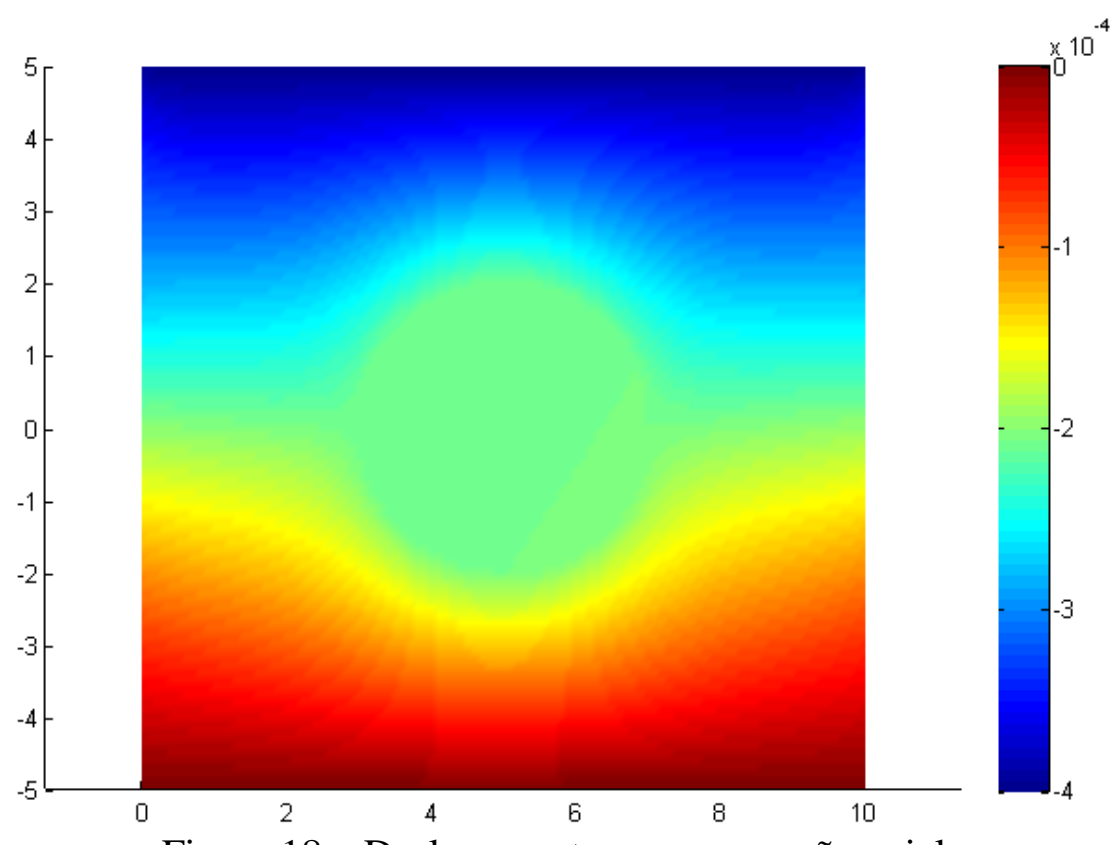

Figura 18 - Deslocamentos a compressão axial. 
O modelo com inclusão circular foi desenvolvido também com diferentes nuvens de partículas para se verificar a eficiência do método numérico utilizado. A Figura 19 ilustra o modelo com 400 partículas e 3600 partículas e a Figura 20 ilustra os deslocamentos lineares majorados, quando submetido a um carregamento de compressão axial, para uma melhor visualização de como a presença da inclusão interfere no comportamento do material. Para ambos os casos foi admitida a mesma malha de integração.

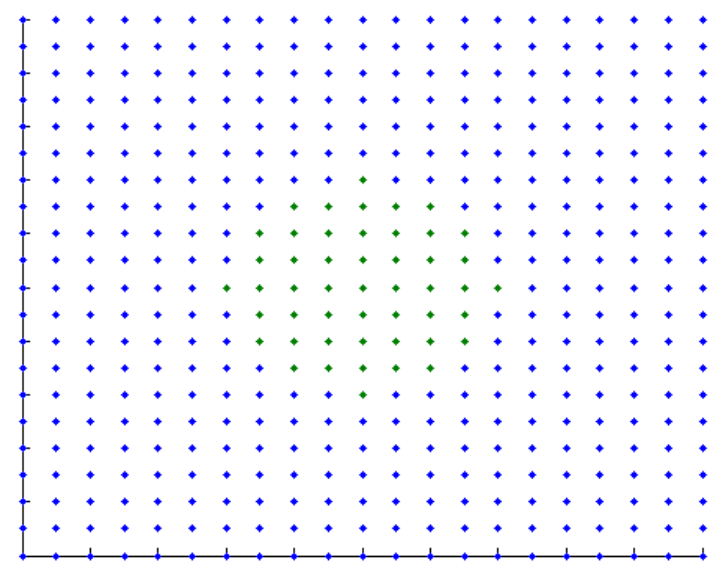

(a)

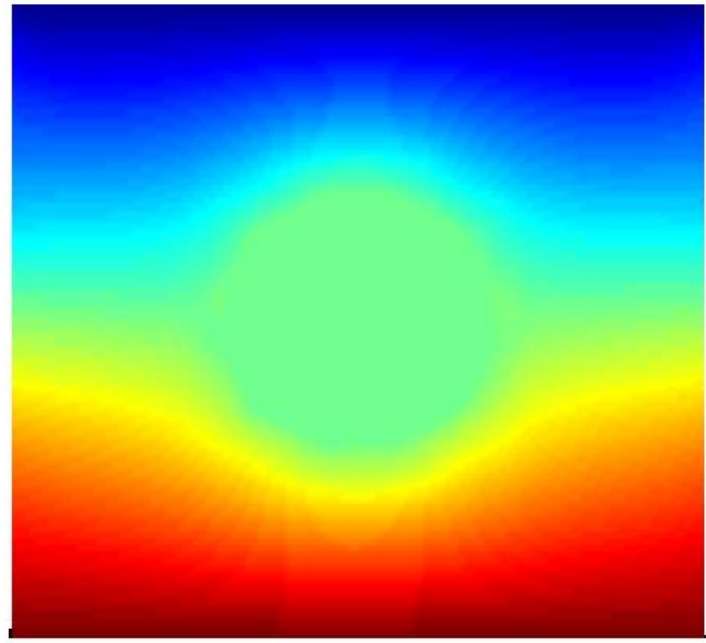

(c)

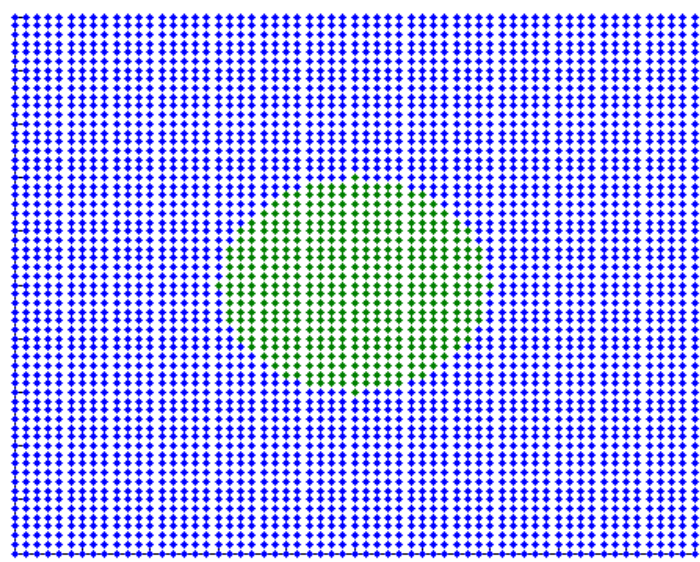

(b)

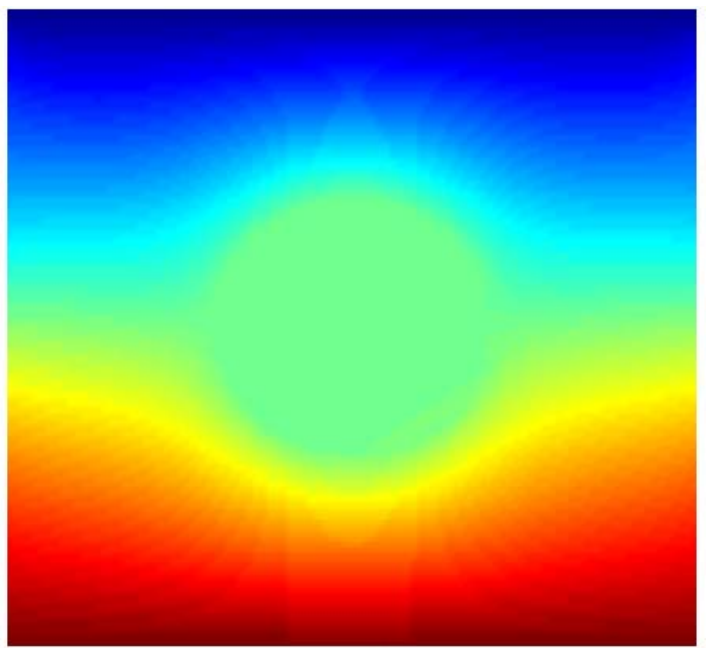

(d)

Figura 19 - (a) Nuvem com 400 partículas, (b) Nuvem com 3600 partículas, (c) Deslocamentos verticais com a nuvem de 400 partículas e (d) Deslocamentos verticais com a nuvem de 3600 partículas. 


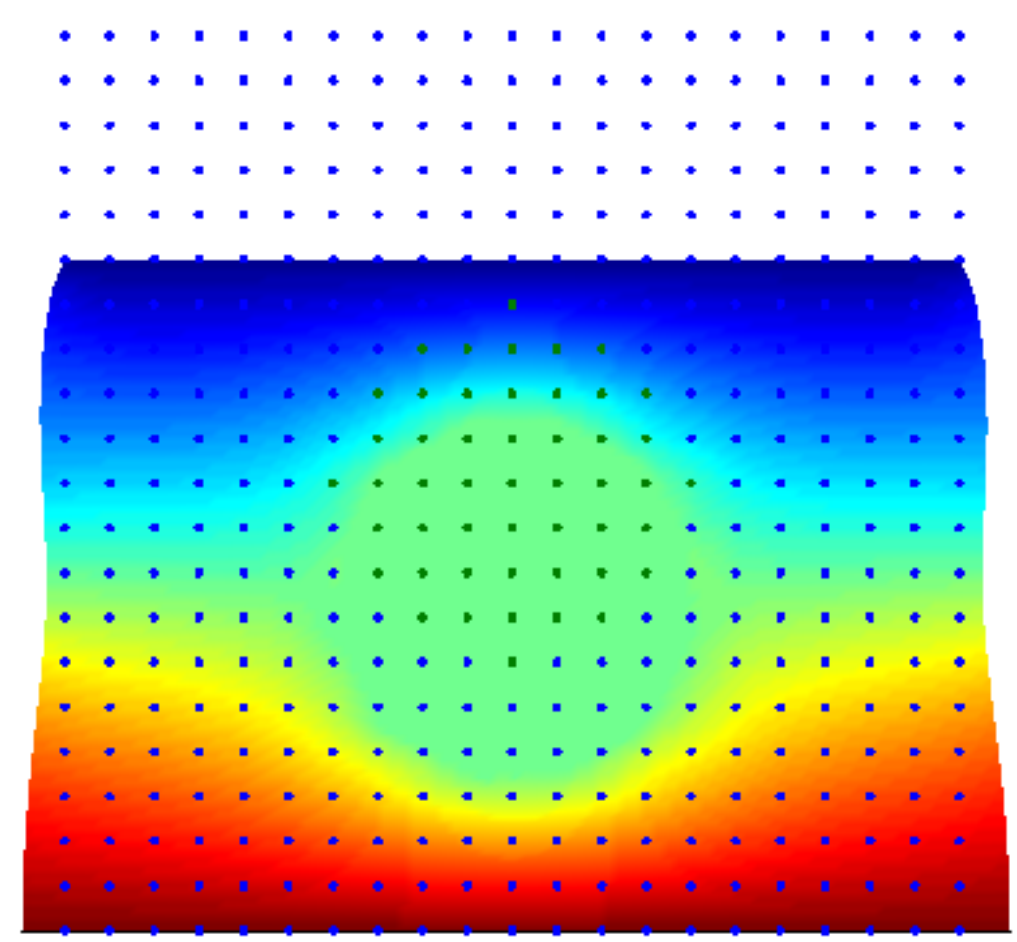

Figura 20 - Deslocamento linear majorado.

Os resultados das análises seguintes são de modelos com inclusão elíptica nas possíveis localizações definidas no domínio e nas fronteiras. As distribuições dos agregados são aleatórios ao longo do material e se fez necessário simular os casos dos agregados presentes nas condições de contorno. Esses casos de inclusões presentes nas condições de contorno são para melhor representarmos a célula que irá abranger o comportamento estatístico da heterogeneidade do concreto. A Figura 21 ilustra os deslocamentos verticais obtidos nos modelos quando submetidos à compressão axial. Nestes exemplos foi admitida a força prescrita na fronteira superior. 


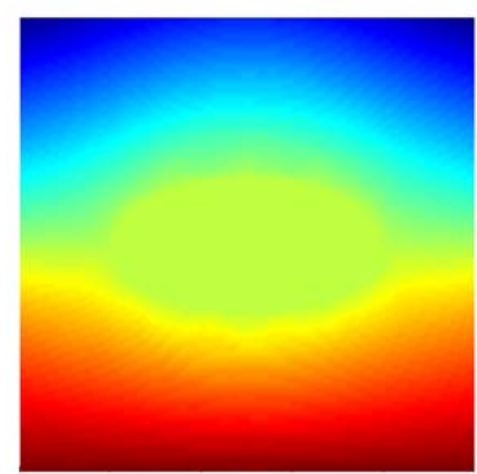

(a)

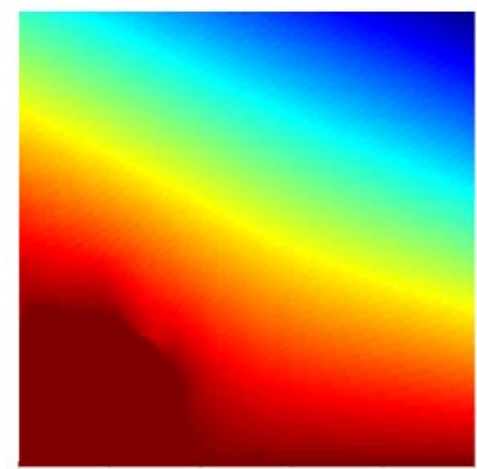

(c)

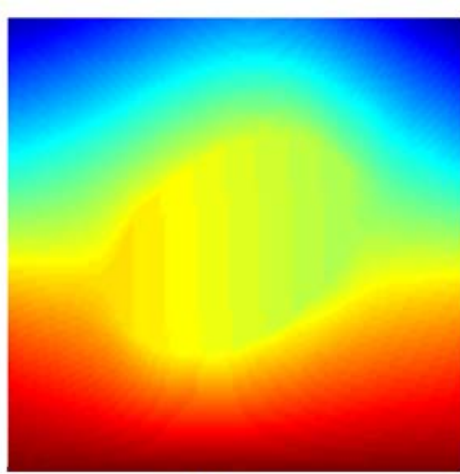

(b)

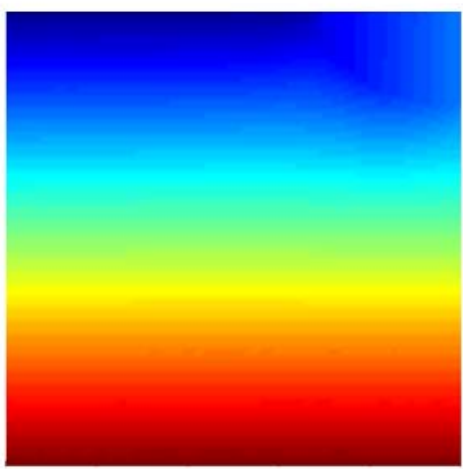

(d)

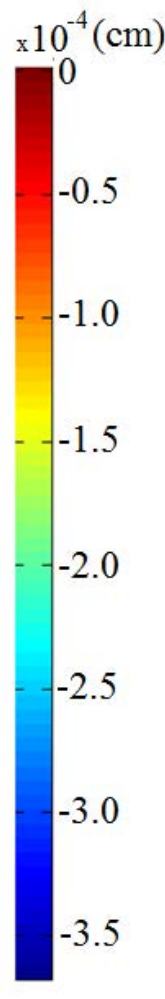

Figura 21 - Deslocamentos verticais. (a) e (b) Inclusão no domínio, (c) e (d) Inclusão na fronteira essencial e natural, respectivamente.

\subsection{Modelo De Material Heterogêneo Com Várias Inclusões}

Nesta etapa desenvolveu-se modelos com varias inclusões simulando a presença do agregado graúdo. A Figura 22 ilustra campo dos deslocamentos quando apresentam mais de uma inclusão. 


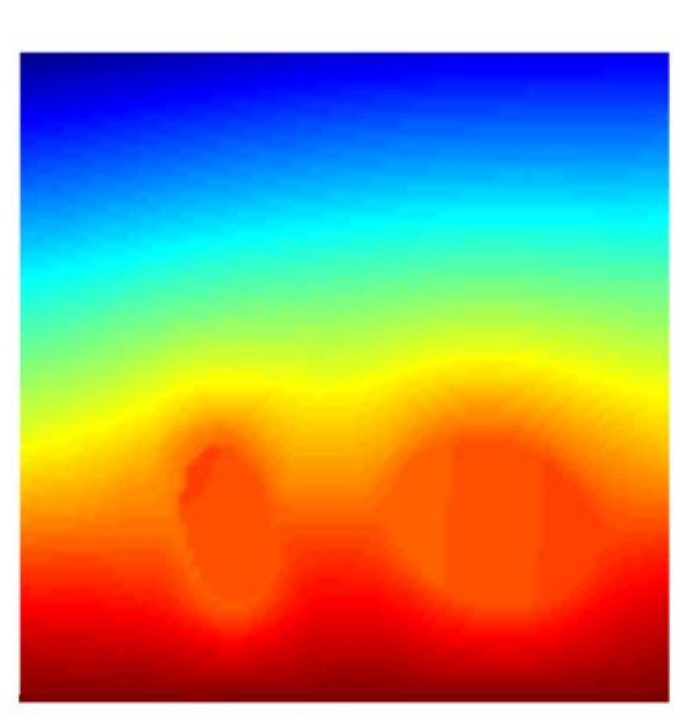

(a)

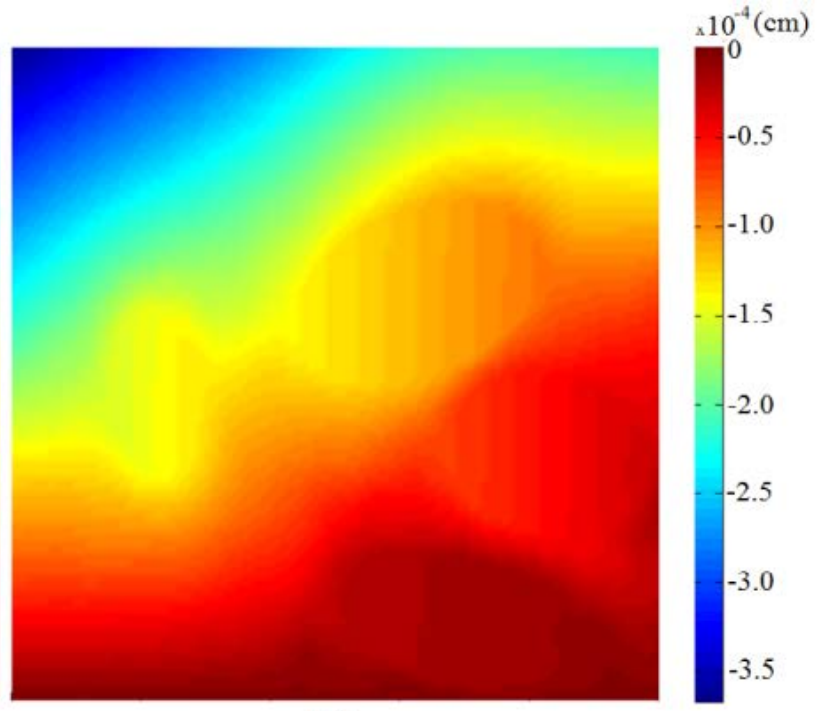

(b)

Figura 22 - (a) Deslocamentos verticaisde modelo com duas inclusões e (b) Deslocamentos verticais de modelo com quatro inclusões.

Para se obter modelos que aproximem do comportamento do concreto é necessário realizar estudo estatístico admitindo uma determinada fração de agregado distribuída aleatoriamente no domínio e com diferentes tamanhos adotados para o RVE. O menor tamanho que apresenta resultados homogeneizados satisfatórios será o RVE adequado para o material.

Devido a limitações computacionais será admitido um RVE a partir da subdivisão de um corpo de prova coletado no laboratório de materiais. As Figura 23 e Figura 24 ilustram a metodologia adotada para obtenção do modelo com doze agregados espalhados no domínio. 

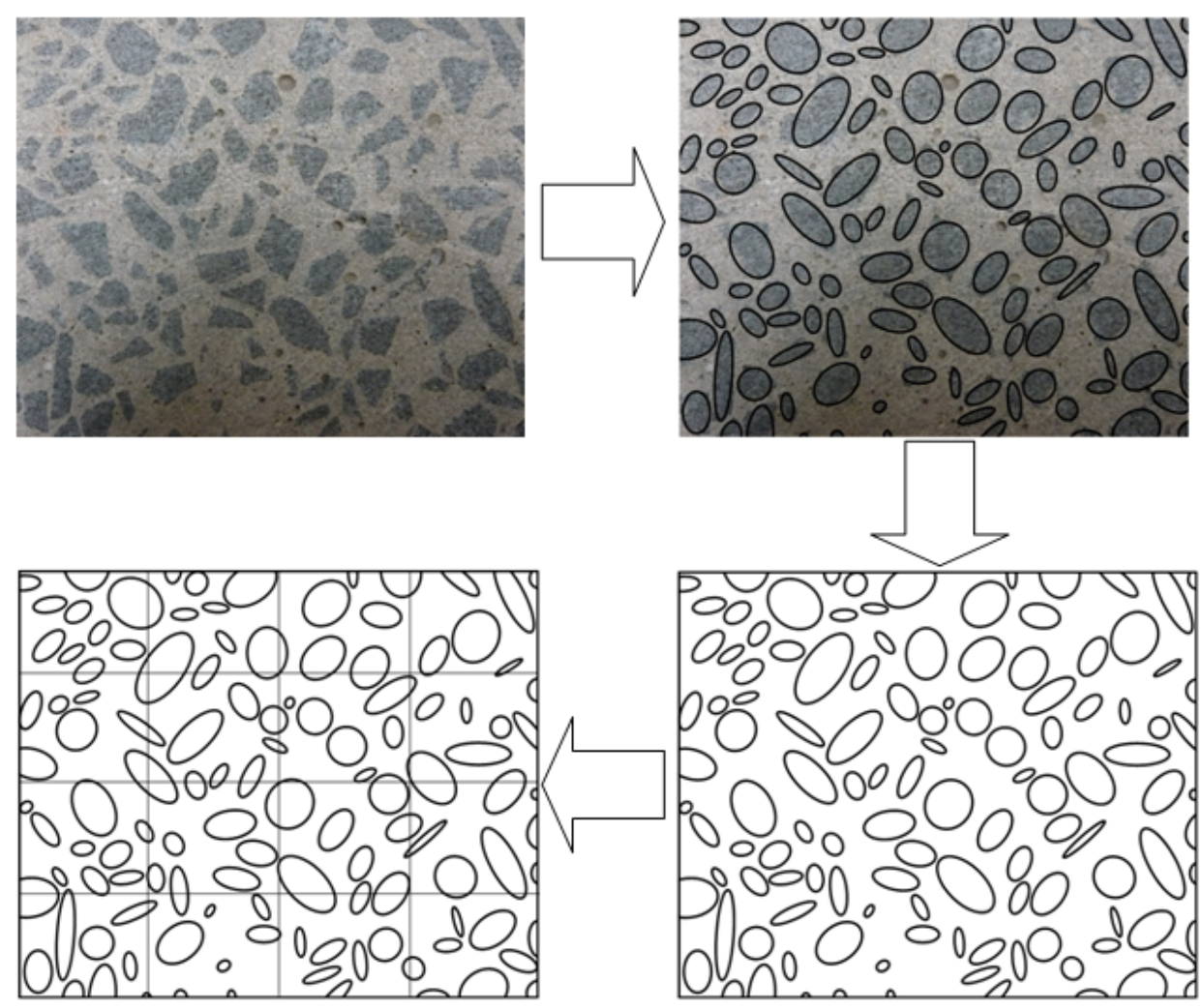

Figura 23 - Obtenção da modelo com várias inclusões.

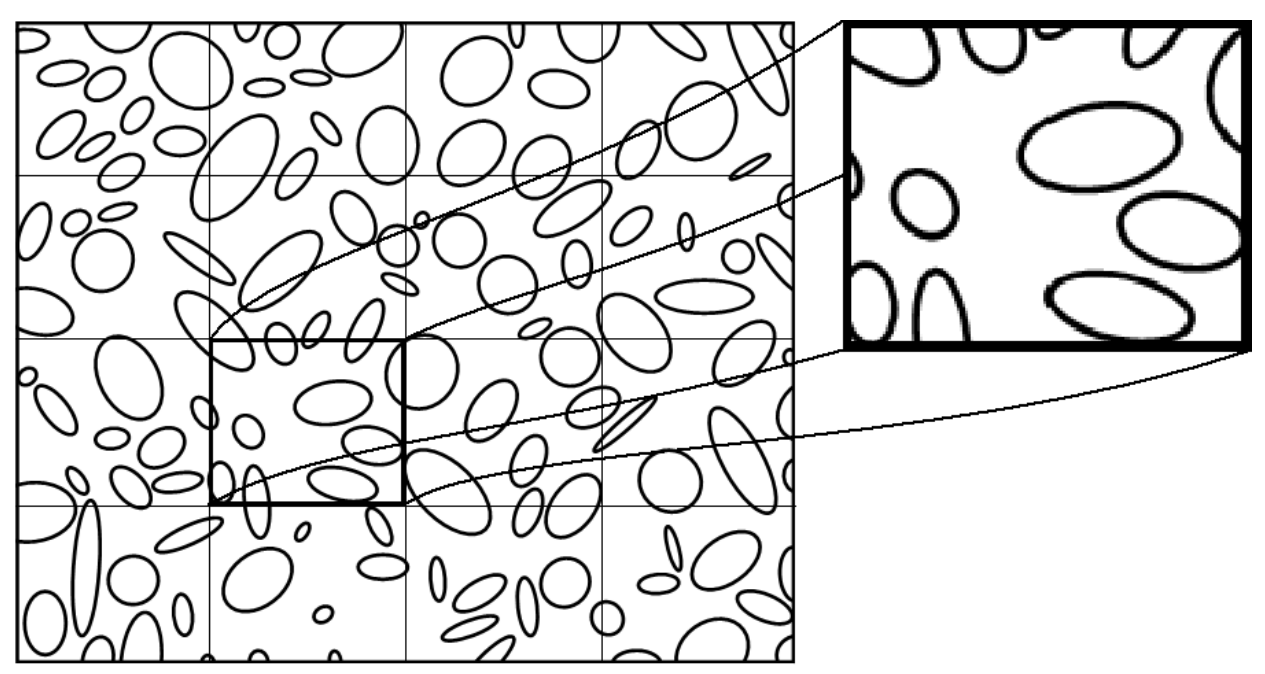

Figura 24 - Detalhe do modelo com várias inclusões. 
As Figura 25 e Figura 26 ilustram o comportamento do modelo computacional quando submetido a compressão axial. Estão apresentados os deslocamentos verticais, a distribuição das tensões verticais e a distribuição das deformações verticais.

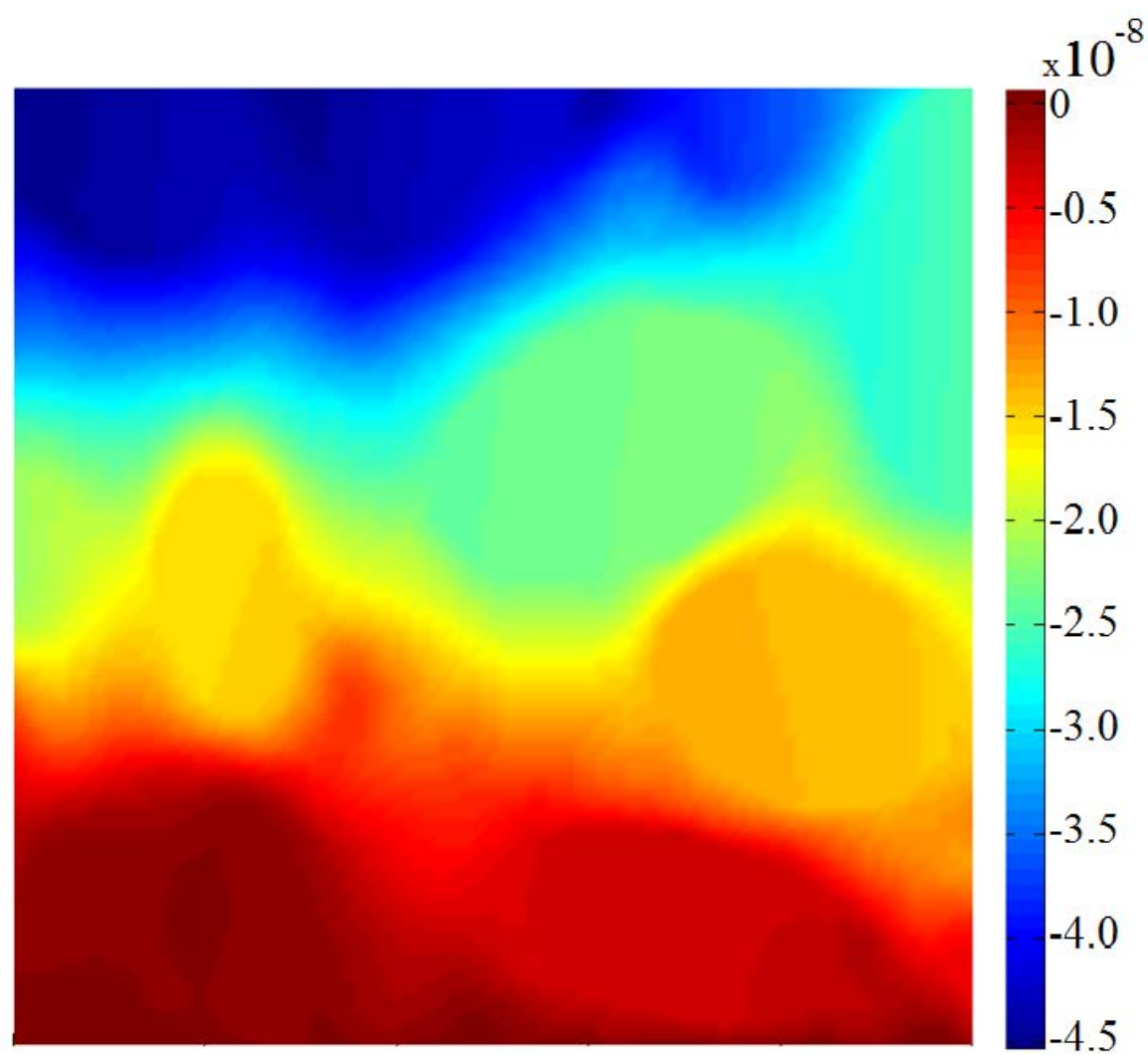

Figura 25 - Deslocamentos verticais

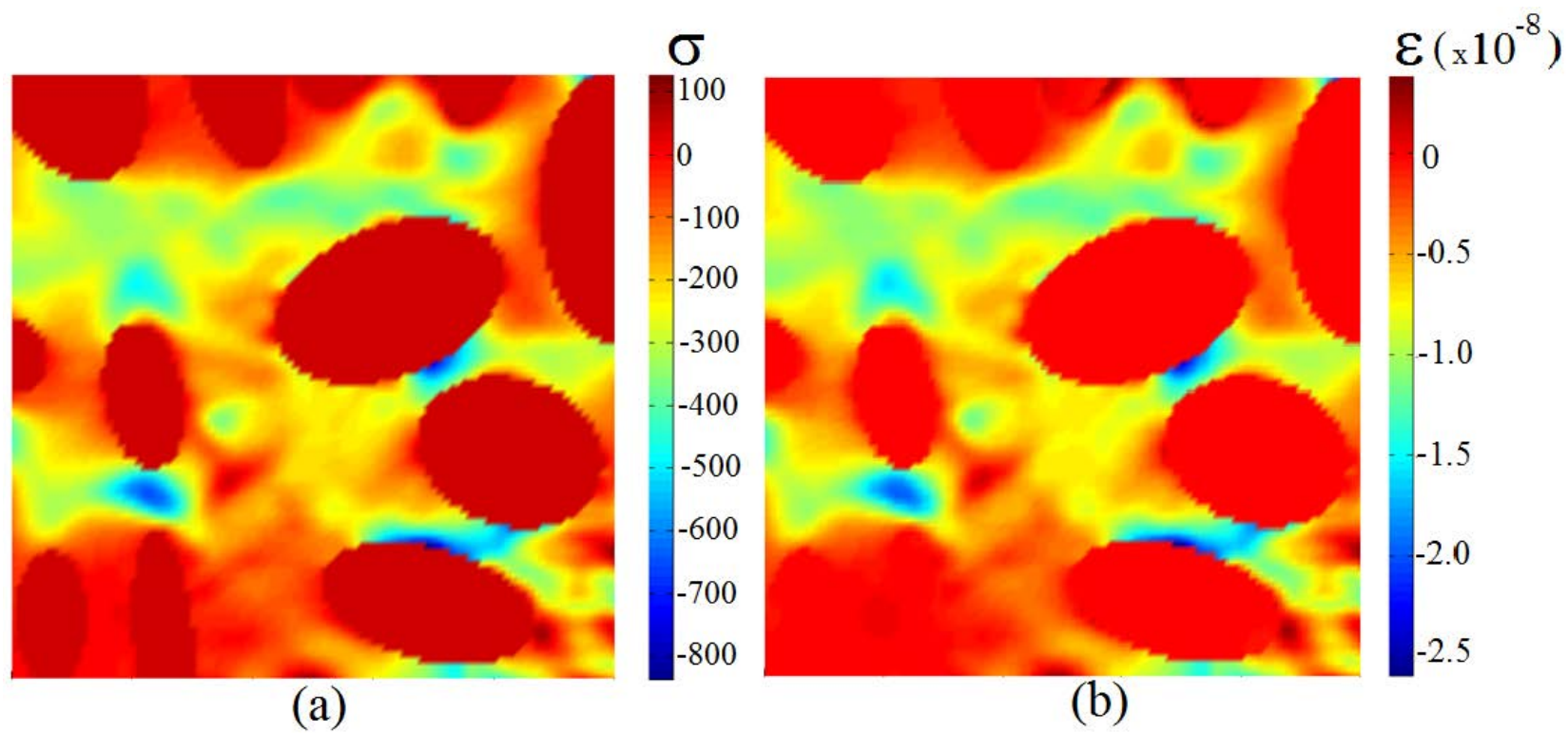

Figura 26 - (a) Distribuição das componentes de tensão normal y e (b) Distribuição das componentes de deformações normais y 


\subsection{Homogeneização Dos Modelos Heterogêneos}

Os modelos heterogêneos desenvolvidos nesta pesquisa são constituídos por duas fases distintas: agregado graúdo e argamassa. Para uma simulação computacional mais próxima da realidade o concreto foi admitido com suas principais fases, porém com o foco na obtenção do módulo de elasticidade do material resultante. A essa metodologia de obtenção das propriedades elásticas a partir de um modelo multifásico é denominado de homogeneização.

Para validação e observação dos resultados obteve - se um modelo heterogêneo com a variação da fração de inclusão. A Tabela 4 e a Figura 27 ilustram os resultados alcançados.

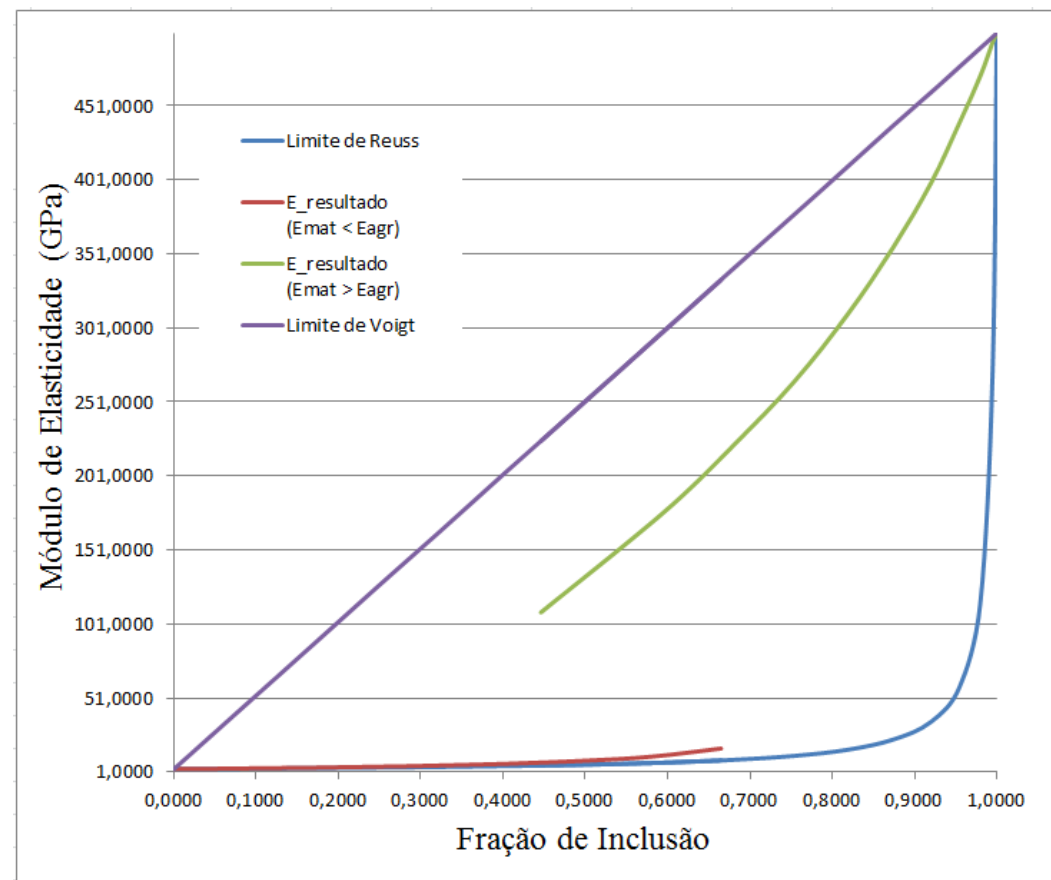

Figura 27 - Comportamento do material homogeneizado. 
Tabela 4 - Resultados obtidos no processo de homogeneização

\section{Homogeneização Módulo de Elasticidade Modelo 2D (x10e3 MPa)}

\begin{tabular}{|c|c|c|c|c|c|}
\hline $\begin{array}{c}\text { Fração do } \\
\text { Material (\%) }\end{array}$ & $\begin{array}{c}\text { Fração do } \\
\text { Agregado (\%) }\end{array}$ & $\begin{array}{c}\text { Limite } \\
\text { de Reuss }\end{array}$ & $\begin{array}{c}\text { E_resultado } \\
\text { (Emat }<\text { Eagr) }\end{array}$ & $\begin{array}{c}\text { E_resultado } \\
(\text { Emat }>\text { Eagr) }\end{array}$ & $\begin{array}{l}\text { Limite de } \\
\text { Voigt }\end{array}$ \\
\hline 1,0000 & 0,0000 & 3,0000 & 3,0000 & & 3,0000 \\
\hline 0,9950 & 0,0050 & 3,0151 & 3,0268 & & 5,4982 \\
\hline 0,9887 & 0,0113 & 3,0341 & 3,0556 & & 8,6209 \\
\hline 0,9799 & 0,0201 & 3,0612 & 3,0984 & & 12,9928 \\
\hline 0,9686 & 0,0314 & 3,0967 & 3,1527 & & 18,6137 \\
\hline 0,9548 & 0,0452 & 3,1413 & 3,2210 & & 25,4838 \\
\hline 0,9384 & 0,0616 & 3,1956 & 3,3068 & & 33,6029 \\
\hline 0,9196 & 0,0804 & 3,2607 & 3,4096 & & 42,9711 \\
\hline 0,8982 & 0,1018 & 3,3377 & 3,5348 & & 53,5884 \\
\hline 0,8743 & 0,1257 & 3,4282 & 3,6822 & & 65,4549 \\
\hline 0,8190 & 0,1810 & 3,6580 & 4,0664 & & 92,9350 \\
\hline 0,7540 & 0,2460 & 3,9726 & 4,6136 & & 125,4115 \\
\hline 0,6780 & 0,3220 & 4,4103 & 5,4199 & & 162,8844 \\
\hline 0,5930 & 0,4070 & 5,0395 & 6,6582 & & 205,3538 \\
\hline 0,4970 & 0,5030 & 5,9957 & 8,7386 & & 252,8194 \\
\hline 0,4190 & 0,5810 & 7,0988 & 11,4405 & & 291,6976 \\
\hline 0,3350 & 0,6650 & 8,8436 & 16,8681 & & 333,3862 \\
\hline 0,5540 & 0,4460 & 5,3874 & & 108,8727 & 224,5741 \\
\hline 0,4070 & 0,5930 & 7,3045 & & 175,3938 & 297,6462 \\
\hline 0,3220 & 0,6780 & 9,2090 & & 220,4754 & 340,1156 \\
\hline 0,2460 & 0,7540 & 11,9606 & & 264,7826 & 377,5885 \\
\hline 0,1810 & 0,8190 & 16,1403 & & 310,3943 & 410,0650 \\
\hline 0,1260 & 0,8740 & 22,9166 & & 355,3392 & 437,5451 \\
\hline 0,0800 & 0,9200 & 34,9072 & & 398,7872 & 460,0289 \\
\hline 0,0450 & 0,9550 & 58,8610 & & 439,9664 & 477,5162 \\
\hline 0,0200 & 0,9800 & 115,4488 & & 471,2299 & 490,0072 \\
\hline 0,0050 & 0,9950 & 272,8168 & & 494,0127 & 497,5018 \\
\hline 0,0000 & 1,0000 & 500,0000 & & 500,0000 & 500,0000 \\
\hline
\end{tabular}

A Figura 28 ilustra o comportamento do material, porém com o eixo das ordenadas em escala logarítmica, para uma melhor visualização dos resultados. Observa - se que o comportamento quando a matriz tem maior rigidez que a inclusão, difere de quando a inclusão 
apresenta maior rigidez. Isso pode ser observado entre os tipos de concreto convencional e concreto leve.

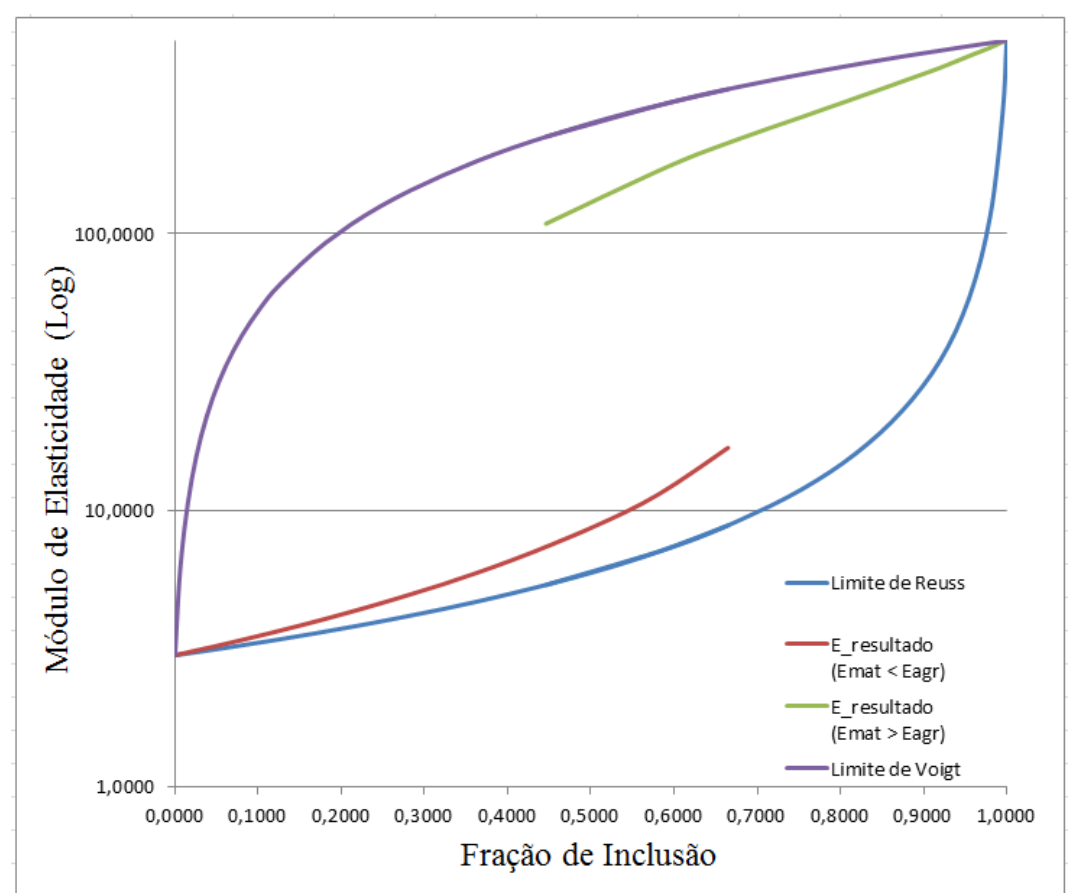

Figura 28 - Comportamento do módulo de elasticidade homogeneizado

\subsection{Método Iterativo de Newton - Raphson}

O método de Newton - Raphson na formulação considerada em mecânica dos sólidos é um método iterativo em que se pesquisa a solução para cada incremento de carga recorrendo a um processo iterativo. O sistema de equações a resolver tem uma solução aproximada. Para se obter uma solução mais próxima da solução exata é necessário considerar uma correção ao vetor deslocamentos. O critério de convergência foi estabelecido em função da norma euclidiana dos resíduos dos esforços virtuais e com o erro admitido de $10^{-2}$.

\subsubsection{Evolução Do Dano No Elemento Representativo}

Inicialmente observou-se o comportamento da evolução do dano na argamassa de um modelo com apenas uma inclusão. A Figura 29 ilustra a evolução obtida neste modelo e a Figura 30 o que está previsto na literatura. Observa-se qualitativamente que os resultados do comportamento da danificação apresentou resultados conforme o previsto. 
Nos modelos, a danificação foi admitida apenas na argamassa devido ser o comportamento dos concretos convencionais por apresentarem agregados graúdos com resistências maiores. Neste caso foi admitido apenas nas visualizações o fator de 1.1 de danificação para os agregados, fora da escala de danificação que vai de 0 (material integro) a 1 (material completamente danificado).

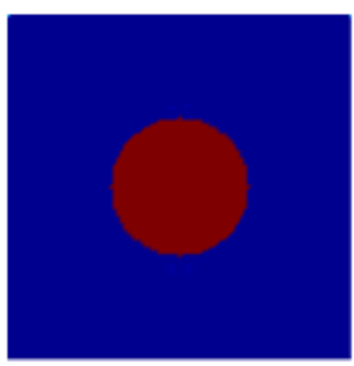

(a)

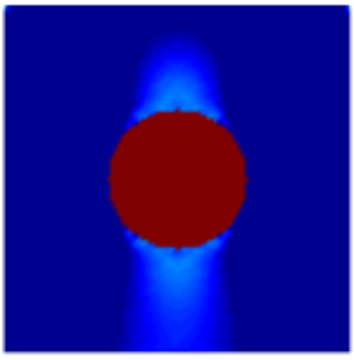

(d)

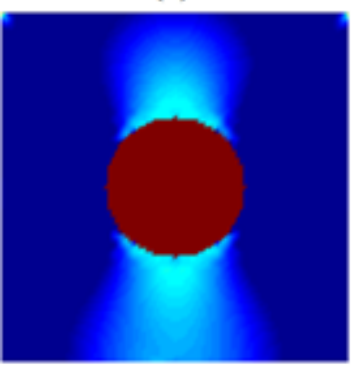

(g)

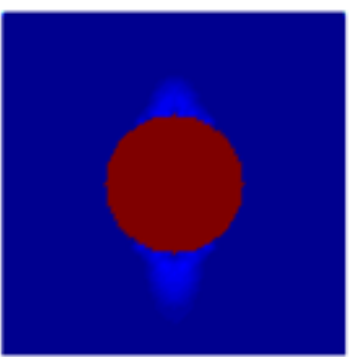

(b)

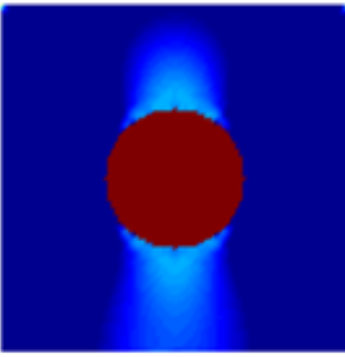

(e)

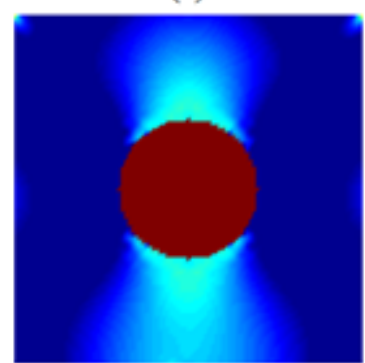

(h)

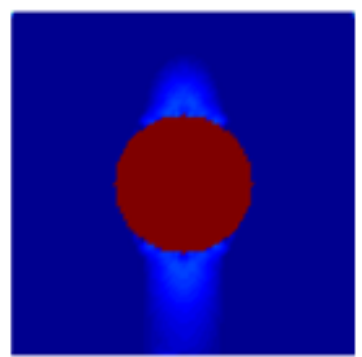

(c)

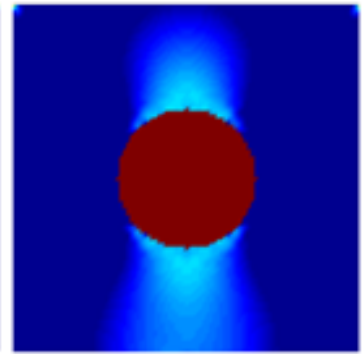

(f)

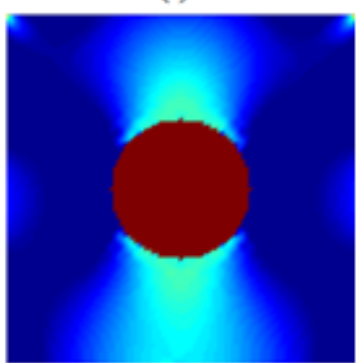

(i)

Danificação

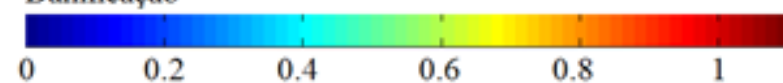

Figura 29 - Evolução da danificação do modelo com uma inclusão. 


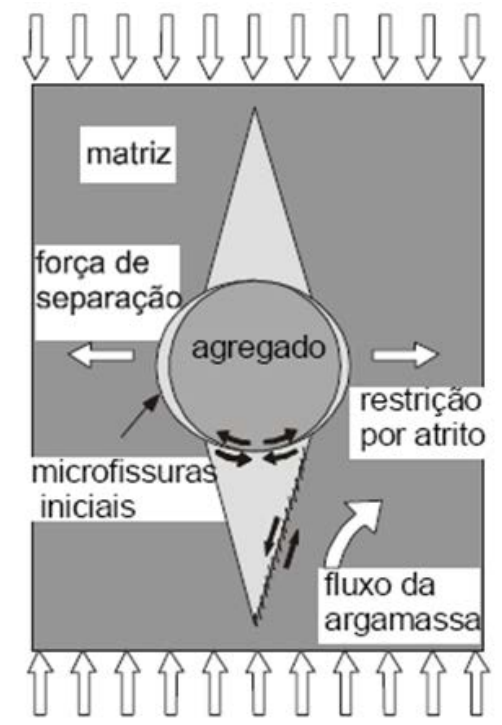

(a)

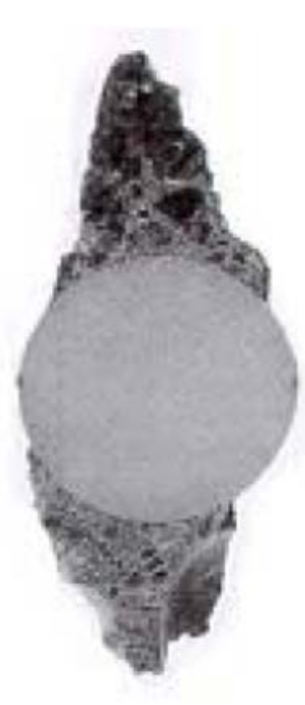

(b)

Figura 30 - Processo de ruptura esperado para o modelo com uma inclusão. [5]

Para a evolução da danificação foi admitido outro modelo computacional com sete inclusões obtidos a partir do elemento representativo A1 ilustrado na Figura 31. Apenas para uma melhor visualização da evolução, foi realizado uma pequena mudança na localização dos agregados 35 e 41 e outro agregado de dimensões muito inferior foi desconsiderado. Ressaltase que o desenvolvimento dos modelos computacionais com mais inclusões, como ilustra os A2, A3 e A4 da Figura 31, não foram implementados computacionalmente apenas por limitações com o equipamento computacional disponível.

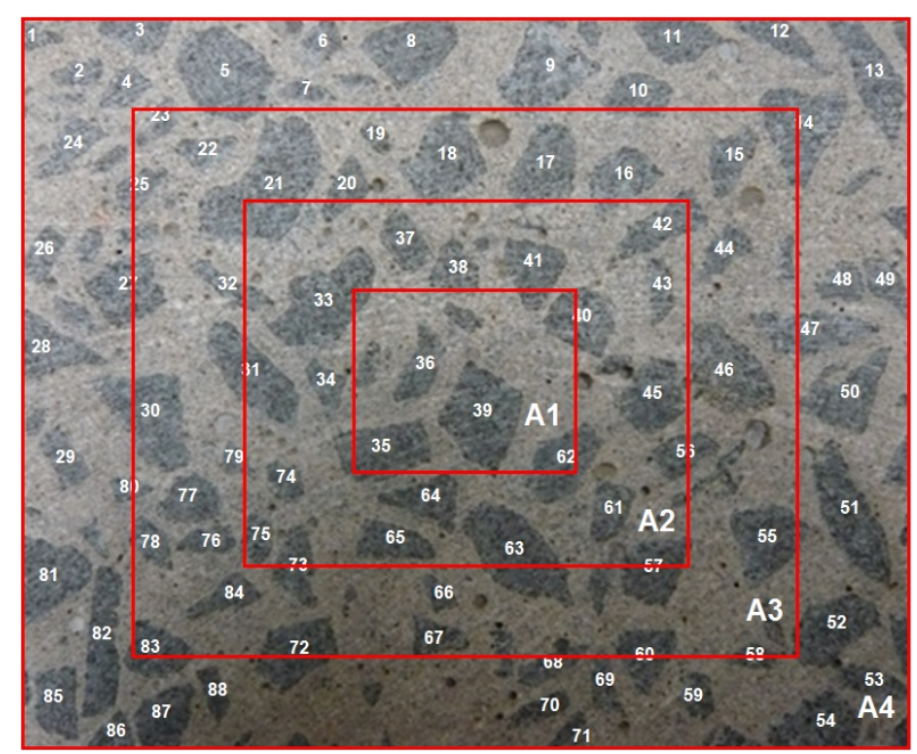

Figura 31 - Redivisão dos elementos representativos no corpo de prova. 
A Figura 32 ilustra a evolução da danificação em oito incrementos de deslocamento prescrito. Observa-se que a localização dos agregados influem no surgimento de caminhos preferenciais de danificação. Vale ressaltar, que a danificação foi admitida apenas na argamassa e a visualização do agregado foi assumido com danificação 1.1 (fora da escala de danificação).

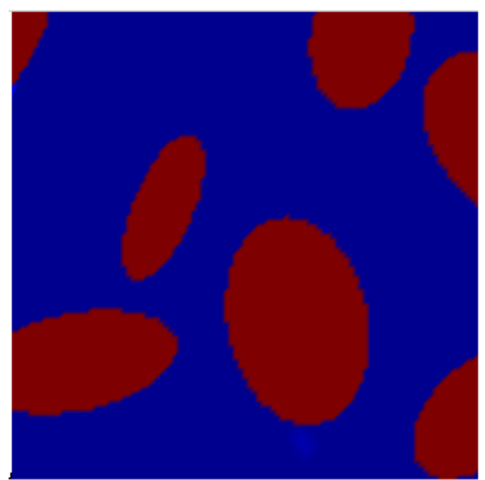

(a)

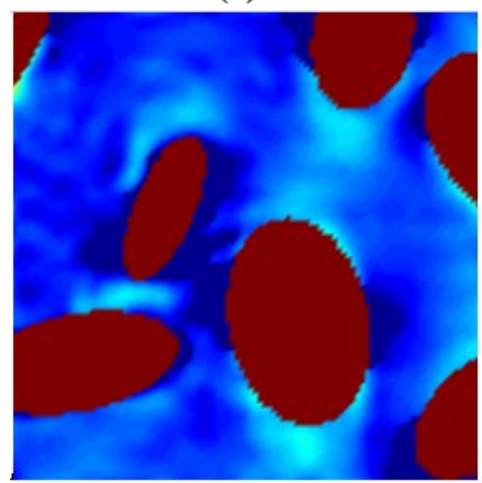

(d)

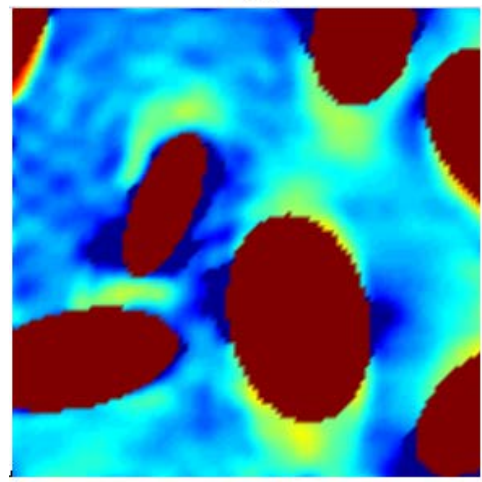

(g)

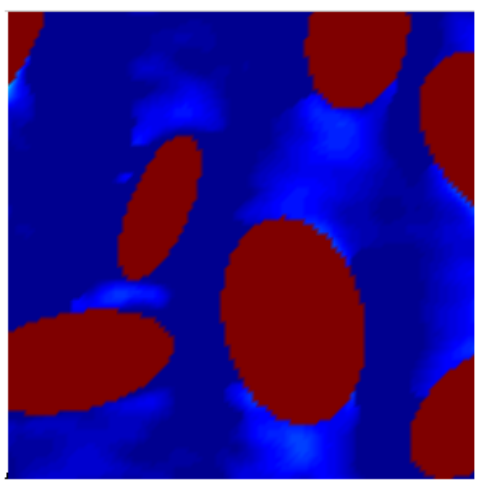

(b)

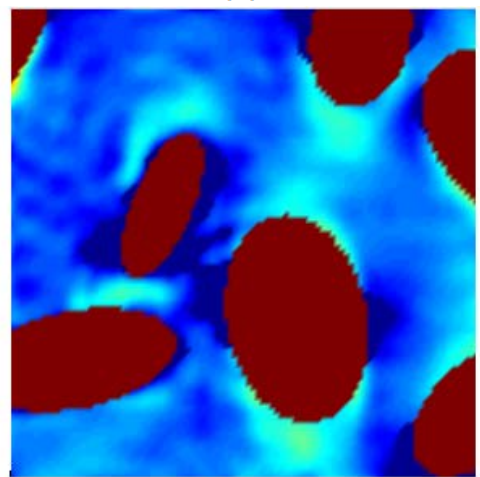

(e)

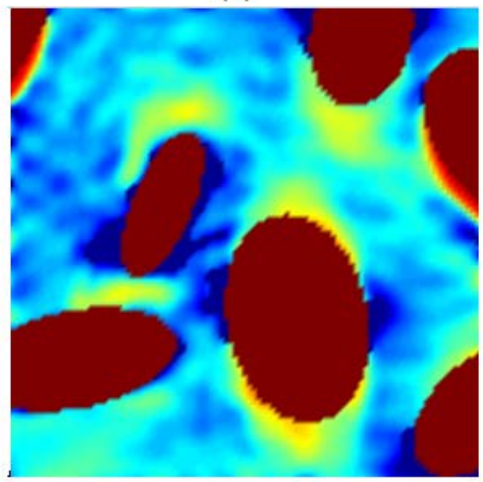

(h)

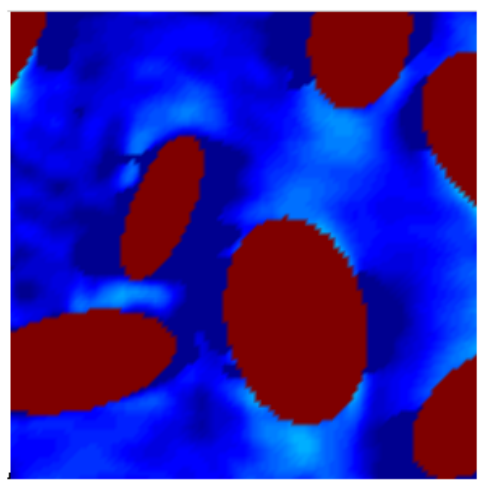

(c)

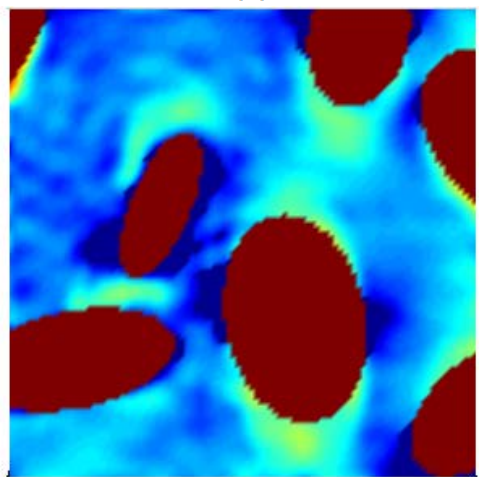

(f)

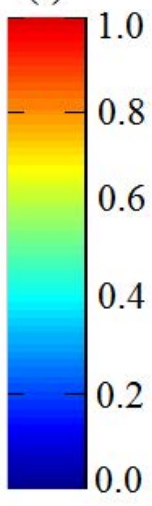

0.0

Figura 32 - Evolução da danificação no modelo com sete inclusões. 


\subsubsection{Convergência Nas Iterações}

O processo de convergência durante as iterações foi obtido admitindo duas matrizes de rigidez:

- Matriz de rigidez elástica

- Matriz de rigidez danificada

Seguindo essa metodologia a Figura 33 ilustra o processo de convergência das iterações e observa-se que quando a matriz de rigidez é danificada o método de Newton encontra mais facilidade na convergência dos resultados. As primeiras iterações não apresentam diferença, porém, a partir da quarta iteração a diferença de diminuição do erro fica cada vez maior.

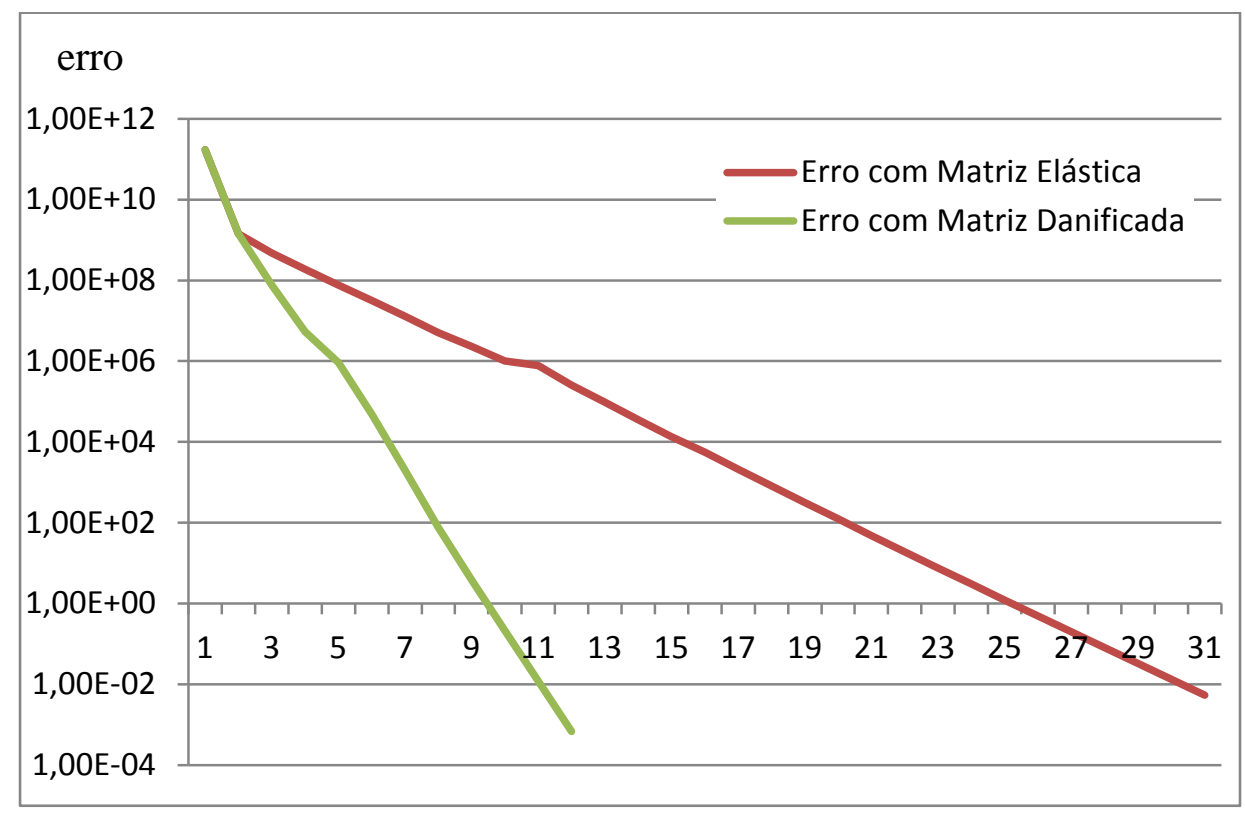

Figura 33 - Convergência com matriz elástica e matriz danificada.

\subsubsection{Homogeneização Dos Modelos Com Dano}

A partir dos incrementos de deslocamento prescrito no ensaio de compressão, obteve-se a evolução da danificação do modelo heterogêneo pelo modelo de Mazars e em cada passo de carga obteve-se a rigidez equivalente do material. Observa-se um decaimento 
não linear da rigidez do material até o colapso do mesmo. A Tabela 5 e Figura 34 ilustram os resultados da rigidez equivalendo do material ao longo dos incrementos.

Tabela 5 - Homogeneização do Material com a evolução do dano Incremento Danificação (\%) Rigidez do Material

\begin{tabular}{|lll|}
\hline $\mathbf{1}$ & 0,00 & (MPa) \\
\hline $\mathbf{2}$ & 0,00 & 36,82 \\
\hline $\mathbf{3}$ & 0,00 & 36,82 \\
\hline $\mathbf{4}$ & 0,00 & 36,82 \\
\hline $\mathbf{5}$ & 0,00 & 36,82 \\
\hline $\mathbf{6}$ & 2,05 & 36,82 \\
\hline $\mathbf{7}$ & 16,47 & 36,80 \\
\hline $\mathbf{8}$ & 29,08 & 36,24 \\
\hline $\mathbf{9}$ & 56,14 & 35,45 \\
\hline $\mathbf{1 0}$ & 82,00 & 34,45 \\
\hline $\mathbf{1 1}$ & 84,70 & 33,29 \\
\hline $\mathbf{1 2}$ & 85,44 & 32,24 \\
\hline $\mathbf{1 3}$ & 85,93 & 31,29 \\
\hline $\mathbf{1 4}$ & 86,28 & 30,41 \\
\hline $\mathbf{1 5}$ & 86,47 & 29,60 \\
\hline $\mathbf{1 6}$ & 86,61 & 28,83 \\
\hline $\mathbf{1 7}$ & 86,73 & 28,09 \\
\hline $\mathbf{1 8}$ & 86,82 & 27,40 \\
\hline $\mathbf{1 9}$ & 86,90 & 26,73 \\
\hline $\mathbf{2 0}$ & 86,96 & 26,09 \\
\hline $\mathbf{2 1}$ & 87,01 & 25,48 \\
\hline & & 24,88 \\
\hline
\end{tabular}




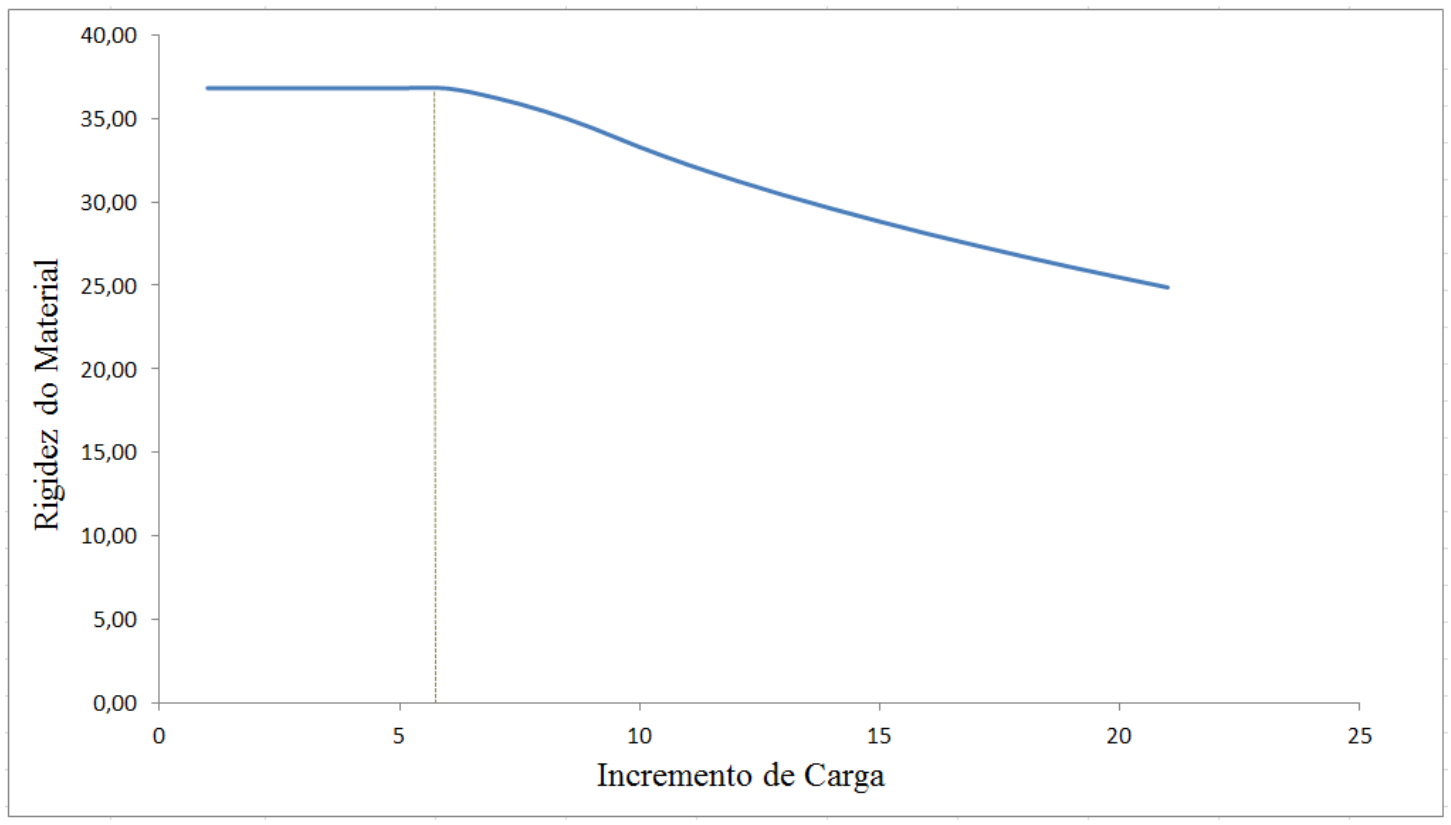

Figura 34 - Evolução da rigidez do material com incremento de deslocamento 
Neste capítulo foram desenvolvidos os modelos computacionais para simular o comportamento do concreto tri - dimensional. O RVE é um cubo de dimensões variadas e com inclusões esféricas e/ou elipsoidais para simular a presença do agregado graúdo dentro do seu domínio. Como foi assumido que é um material com características isotrópicas, é suficiente apenas um teste de carregamento para obtenção do seu comportamento mecânico, neste caso optou - se pela compressão axial.

Como no caso dos modelos bidimensionais, como critério de simplificação, não foram considerados a presença da zona de transição entre o agregado e a argamassa e nem a presença de vazios na argamassa. Para o modelo, o concreto foi assumido como um material com apenas duas fases: agregado graúdo e argamassa.

A metodologia adotada foi dividir em duas etapas:

- Análise linear com uma inclusão;

- Análise linear com várias inclusões.

\subsection{Modelo De Material Heterogêneo Com Uma Inclusão}

Inicialmente foi desenvolvido um modelo cúbico com aresta de 10,00 cm centímetros, com uma inclusão esférica com raio de $2,5 \mathrm{~cm}$. Nos modelos tri - dimensionais procurou-se refinar a nuvem de partículas próximo da interface de mudança de material (agregado - argamassa). A Figura 35 e a Tabela 6 ilustram o modelo com uma inclusão, à nuvem de partículas adotada e as propriedades assumidas na análise. 

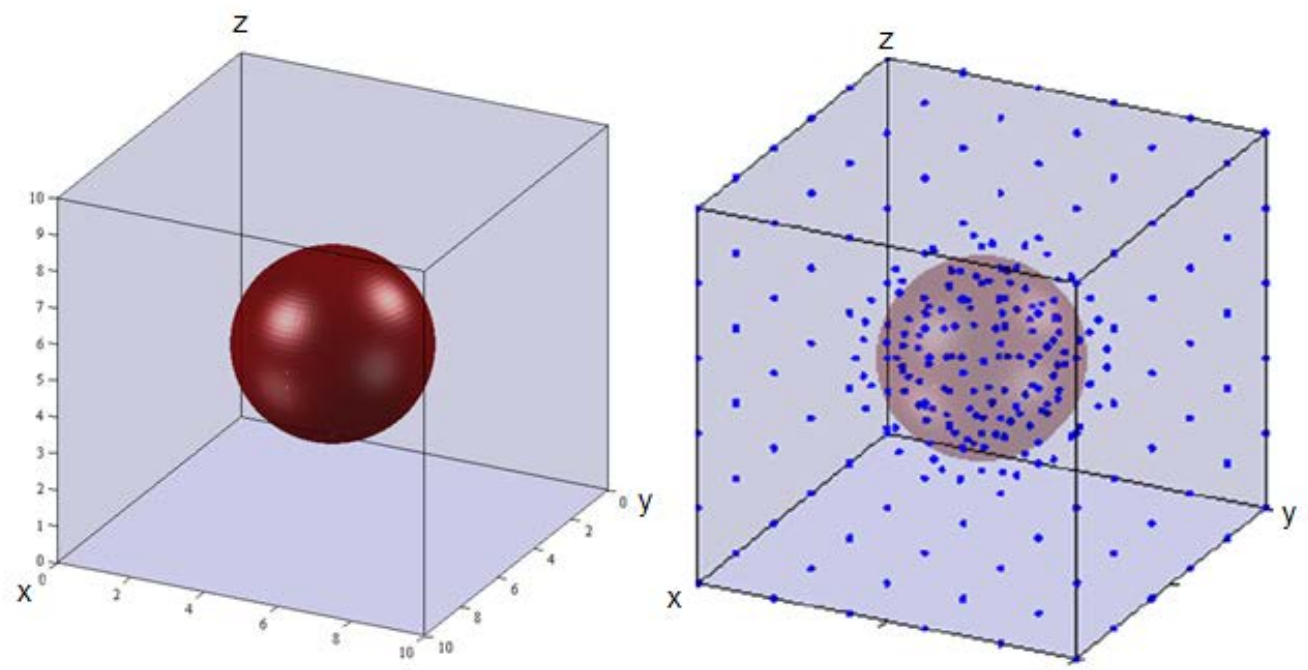

Figura 35 - (a) Modelo 3D com uma inclusão e (b) Nuvem de partículas refinadas na interfase de mudança de material.

Tabela 6 - Descrição das propriedades do modelo

\begin{tabular}{|ccc|}
\hline & Argamassa & Agregado \\
\hline Fração no Volume & $93,488 \%$ & $6,512 \%$ \\
\hline Módulo de Elasticidade & $30 \mathrm{GPa}$ & $5000 \mathrm{GPa}$ \\
\hline Coeficiente de Poisson & 0,30 & 0,13 \\
\hline
\end{tabular}

Os deslocamentos, componentes das tensões e componentes das deformações são apresentados nas Figura 36 a Figura 38. Ressaltou-se a localização da esfera com um corte nas imagens para pode fazer uma melhor análise qualitativa e quantitativa.

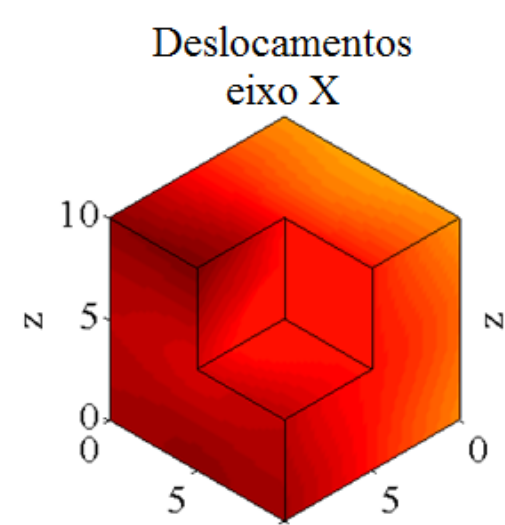

1010
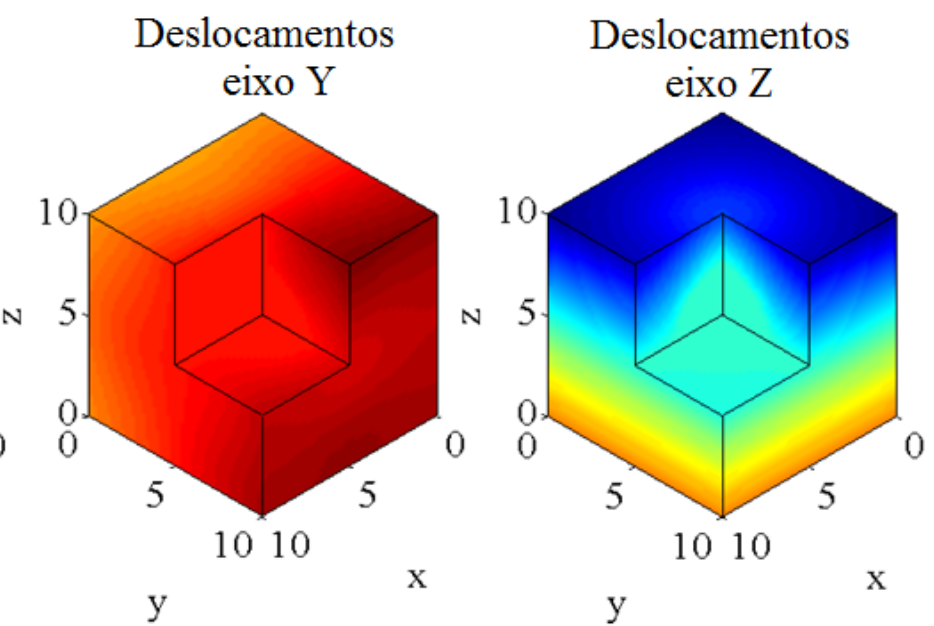

$\mathrm{y}$

Figura 36 - Deslocamentos do modelo com uma inclusão esférica. 

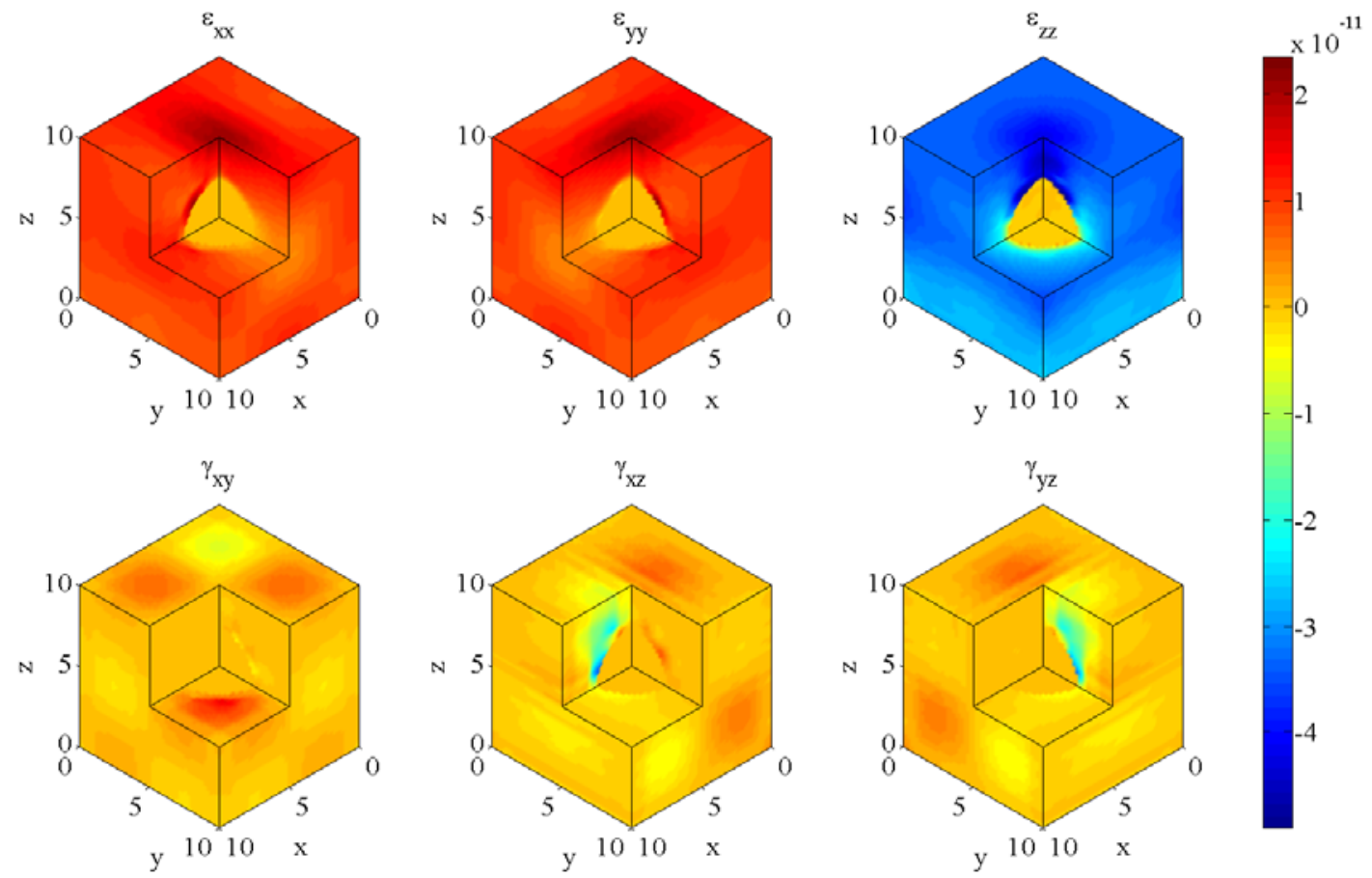

Figura 37 - Deformações do modelo com uma inclusão esférica.
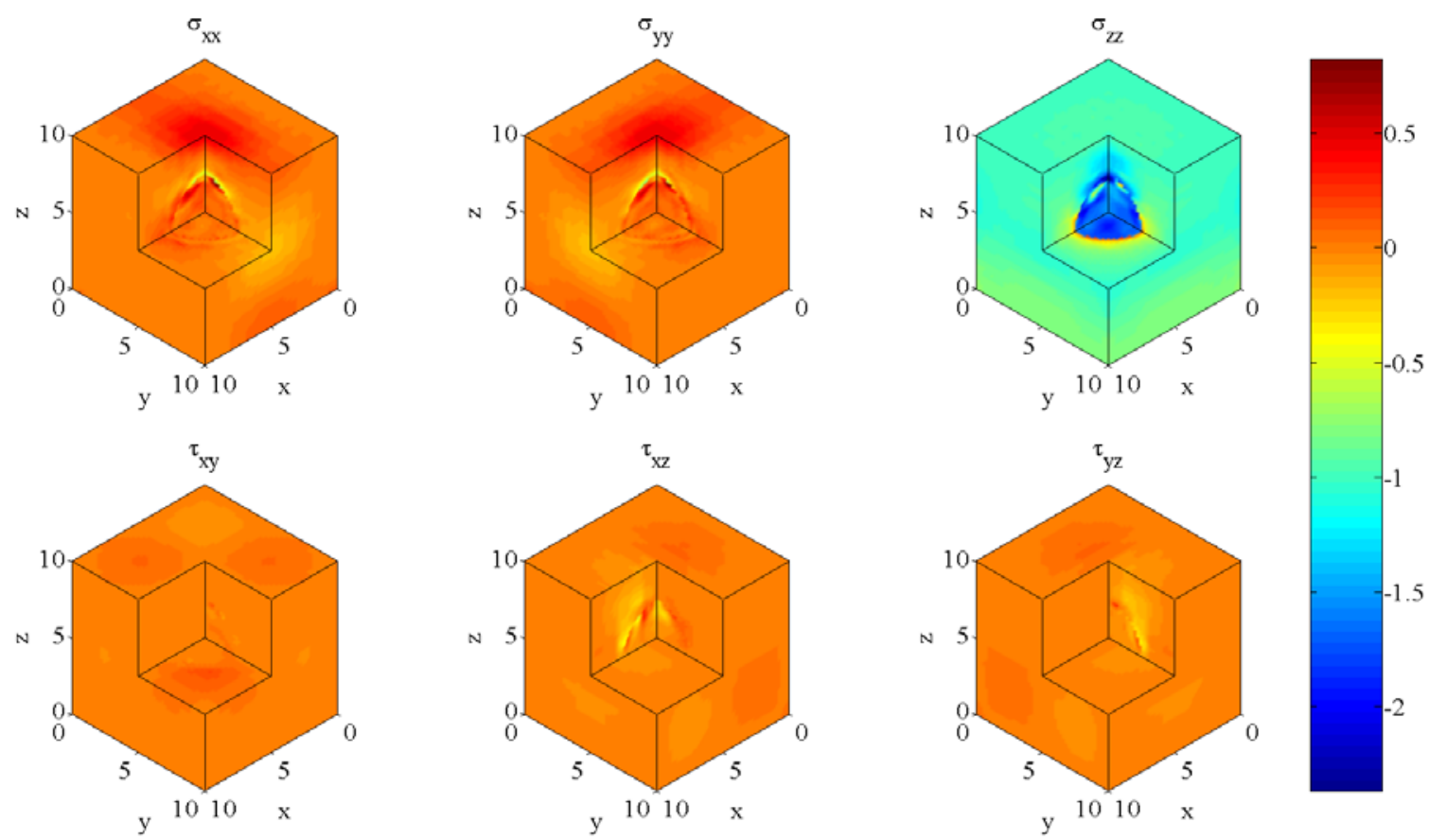

Figura 38 - Tensões do modelo com uma inclusão esférica. 
Como foram formulados os modelos seguindo a metodologia de multiregiões, procurou dar uma maior atenção na interface da inclusão - matriz. A Figura 39 ilustra a distribuição das tensões na interface obtidas na matriz e na inclusão. Observa - se uma pequena descontinuidade numérica dos resultados que podem ser explicados devido ao pouco refinamento da nuvem de partículas e ao baixo grau do polinômio da base do MGLE.
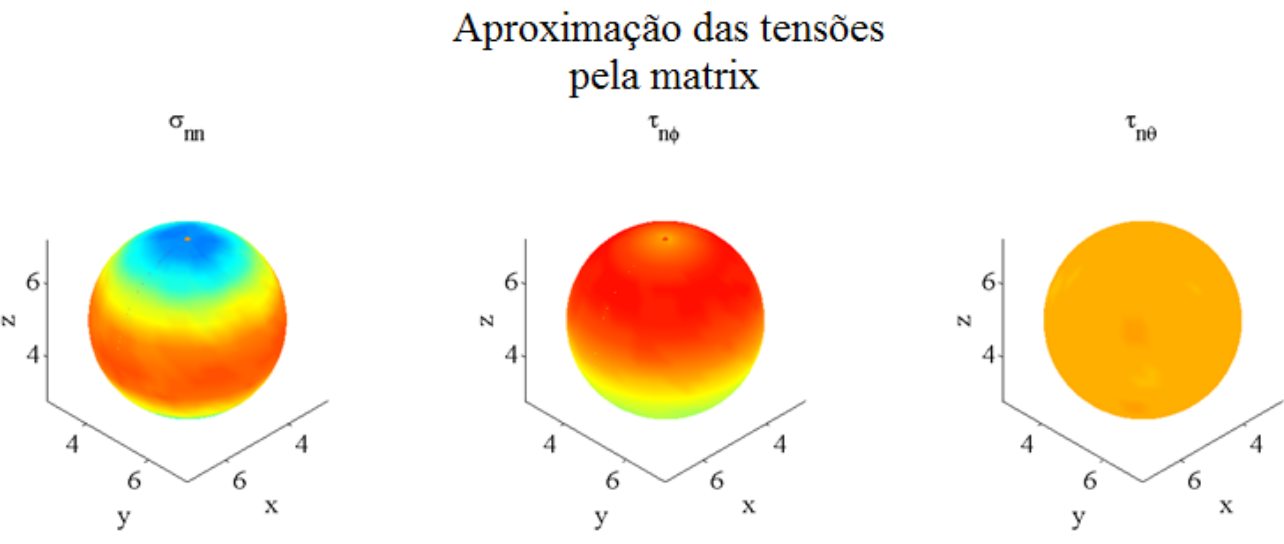

Aproximação das tensões pelo agregado
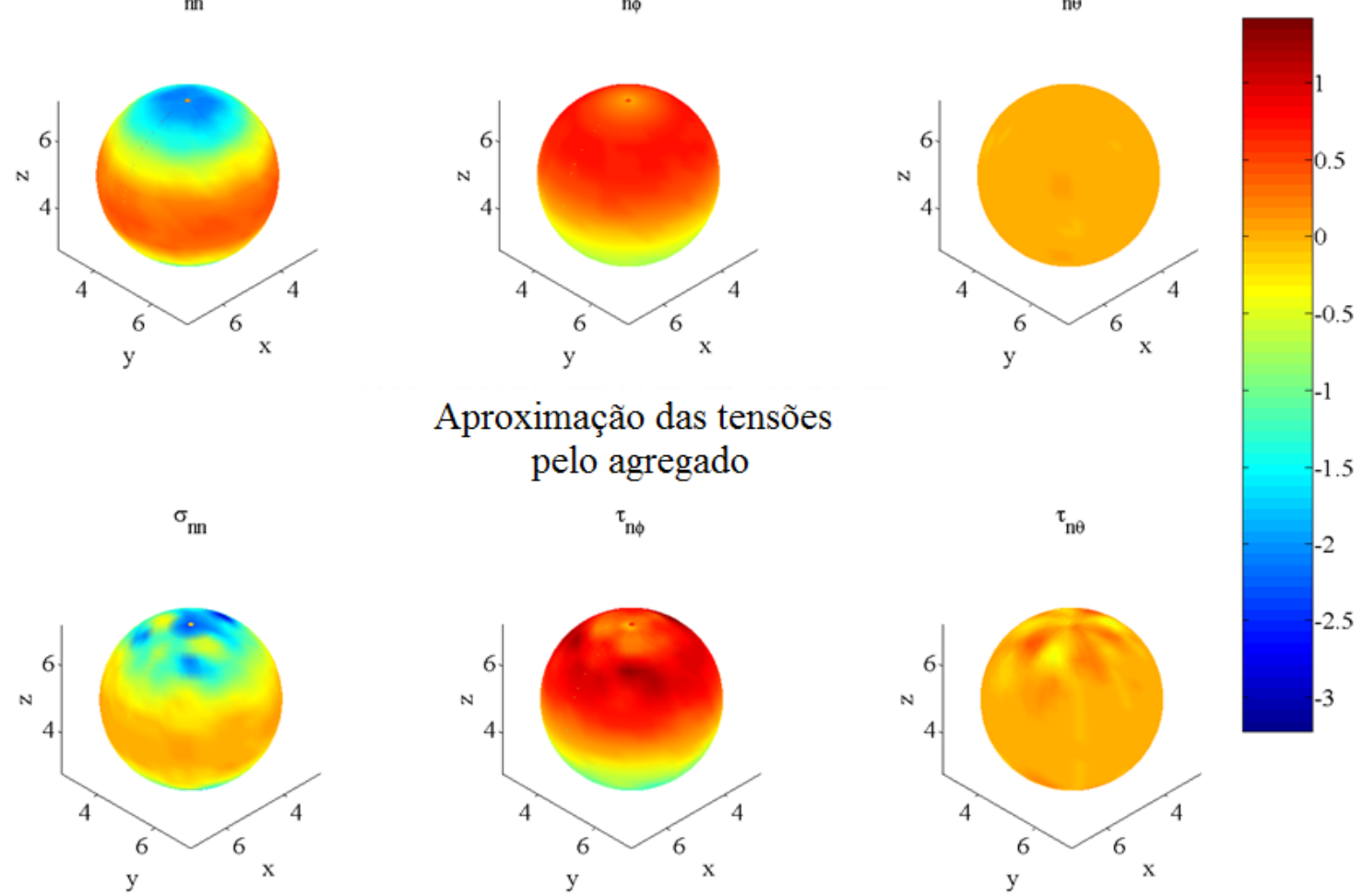

Figura 39 - Tensões na interface da inclusão esférica.

\subsection{Modelo De Material Heterogêneo Com Várias Inclusões}

Para o caso de modelo computacional com várias inclusões foi admitida a presença de 13 (treze) agregados imersos no domínio. Ressalta - se que a presença de agregado graúdo no concreto convencional apresenta proporções em torno de cinquenta por cento (50\%) e com uma curva granulométrica, o que não está representado neste modelo com apenas treze inclusões elipsoidais. 
A ideia desse modelo é ser o protótipo para os demais modelos com proporções adequadas de agregado graúdo imersos na argamassa e com uma curva granulométrica aceitável. A formulação é a mesma, apresentando dificuldades de origem geométrica devido a necessidade de implementar e propor um algoritmo que definirá uma distribuição aleatório dos agregados com diferentes dimensões, diferentes proporções e diferentes curvas granulométricas. Fica este algoritmo como proposta para futuros desenvolvimentos.

A Figura 40 ilustra o modelo na forma de cubo com os trezes agregados dispersos de forma aleatória no seu domínio. Ressalta - se a preocupação em se colocar as formas elipsoidais e rotacionadas também de forma aleatória.

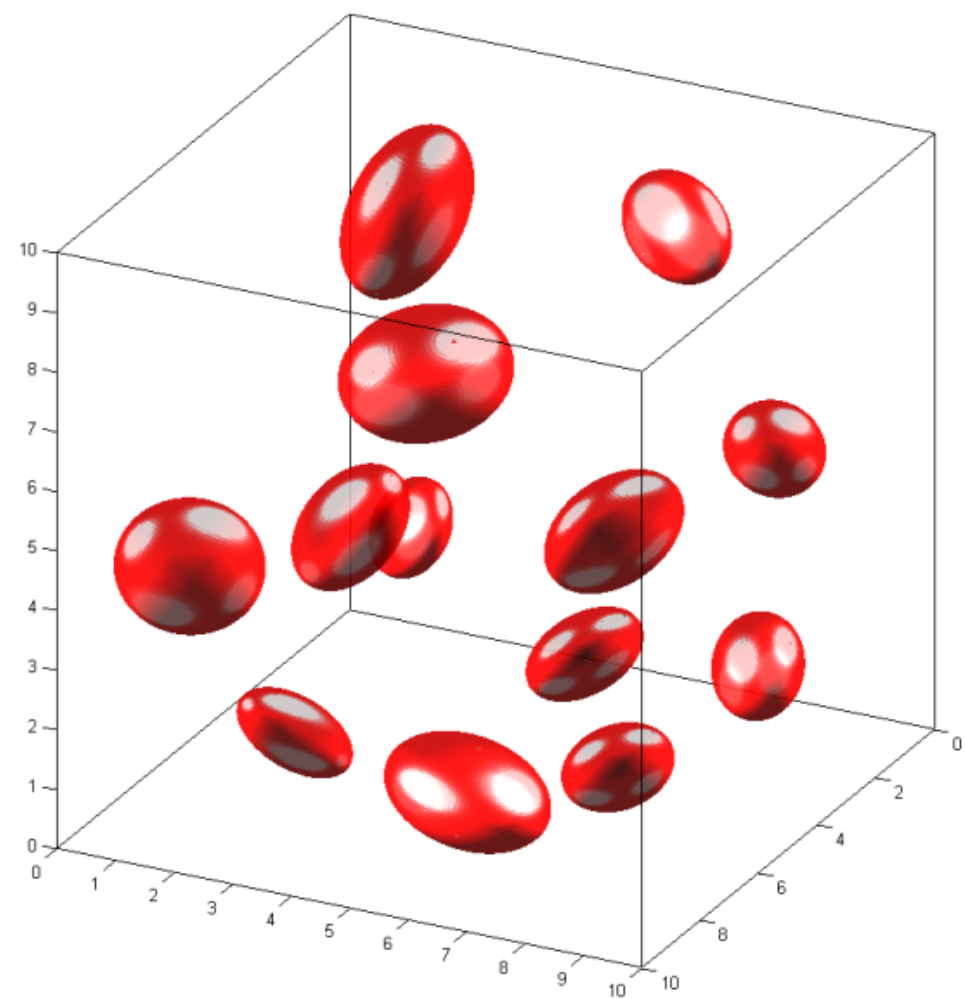

Figura 40 - Modelo com inclusão de 13 agregados.

As Figura 41 e Figura 42 ilustram os deslocamentos obtidos na análise linear do modelo. Observa - se uma continuidade dos resultados o que demonstra coerência nos resultados obtidos. Para uma melhor visualização dos resultados optou - se por três planos perpendiculares entre si e com origem que passasse no centro do modelo. Dessa forma pode se observar o volume do cubo como um todo, porém fica sempre comprometida uma região localizada. 


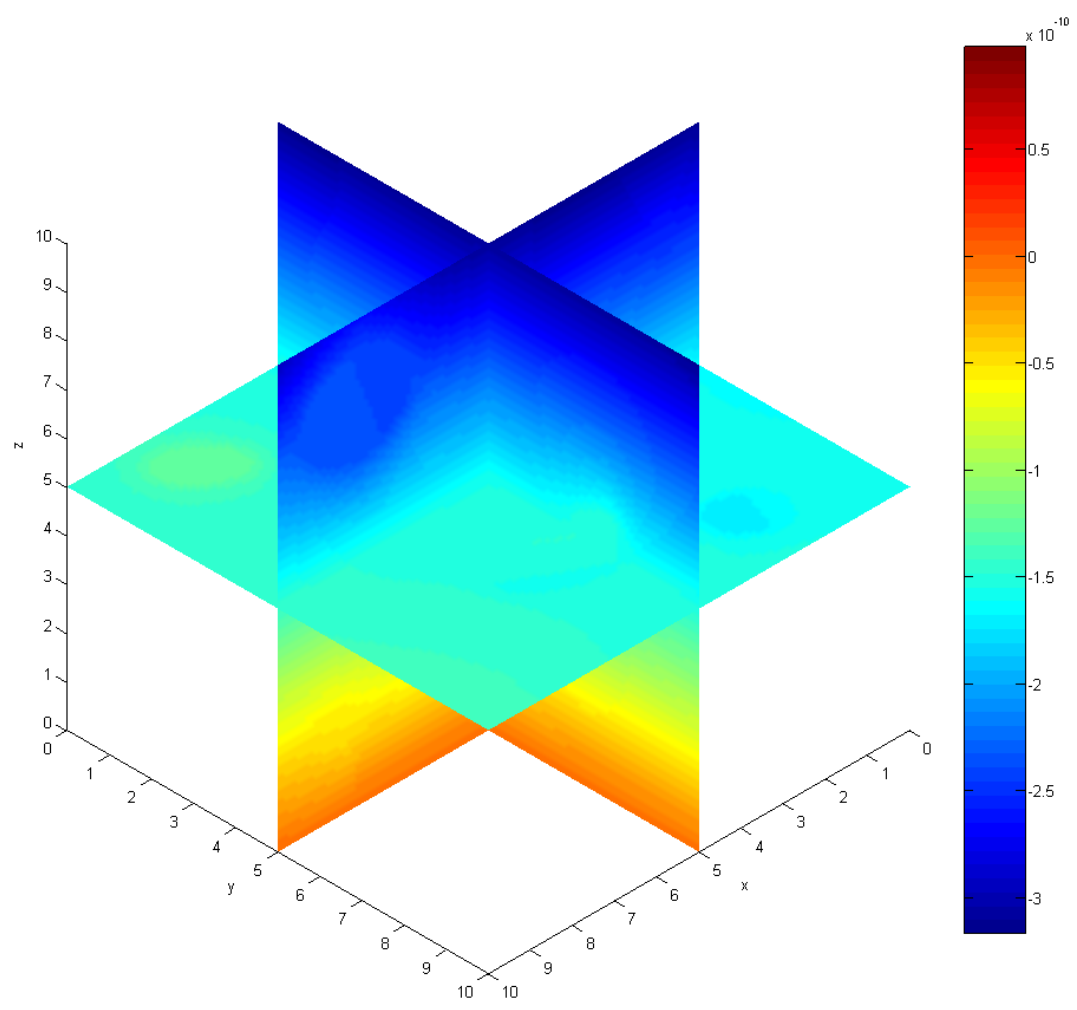

Figura 41 - Deslocamentos no eixo Z do modelo com 13 agregados.

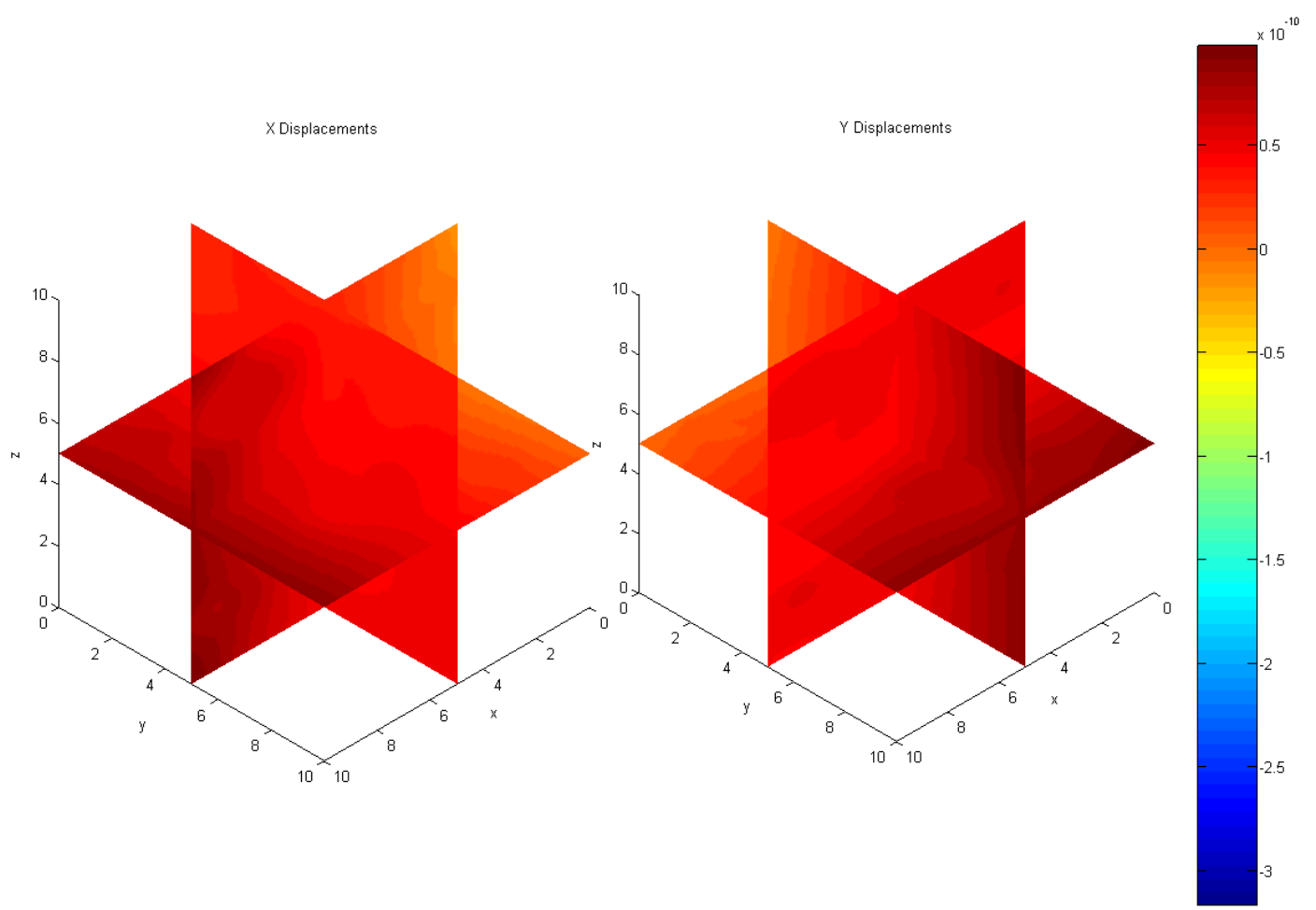

Figura 42 - Deslocamentos nos eixos X e Y do modelo com 13 agregados 
As Figura 43 e Figura 44 ilustram os resultados das tensões e deformações obtidas nesta análise linear tri - dimensional do modelo com treze agregados imersos do domínio da argamassa. Pode - se observar o mapa dos resultados devido à presença das inclusões.
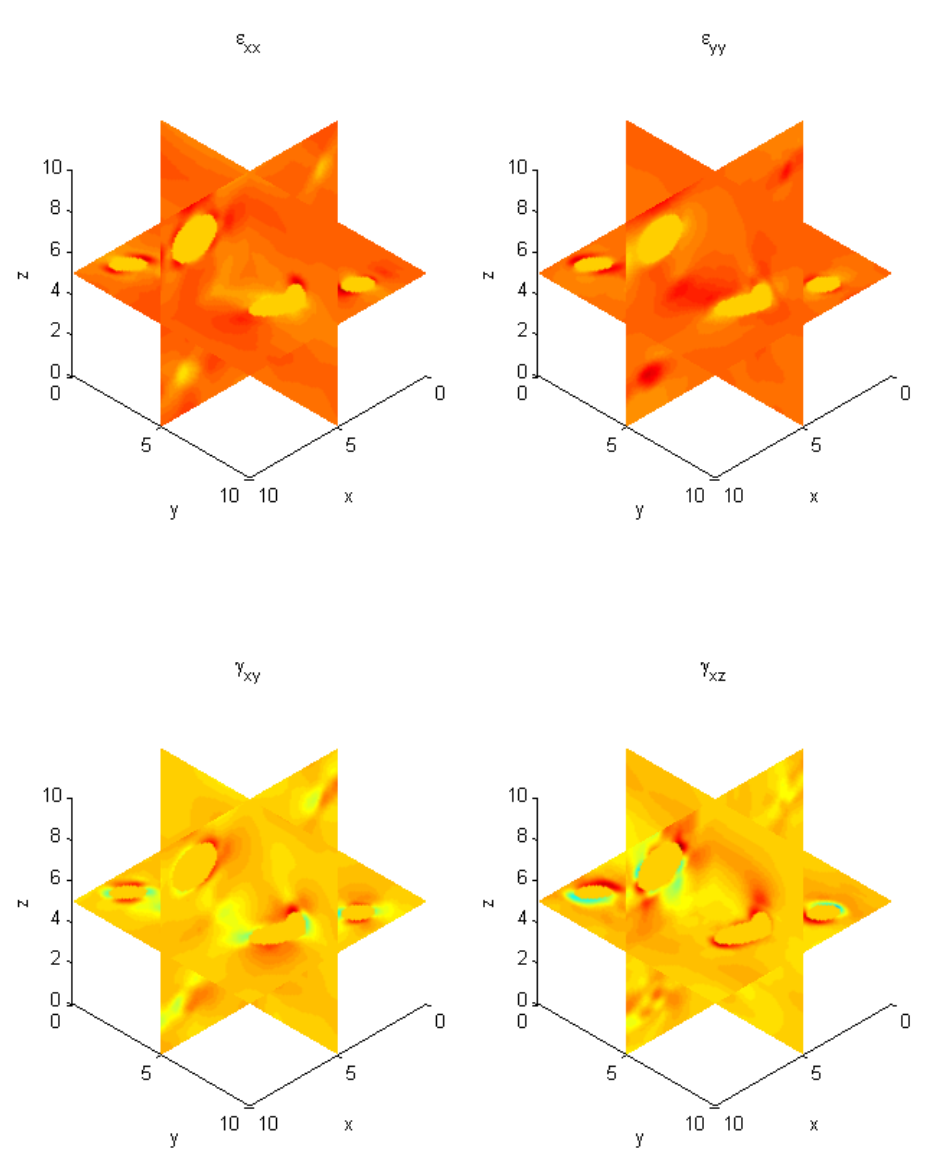
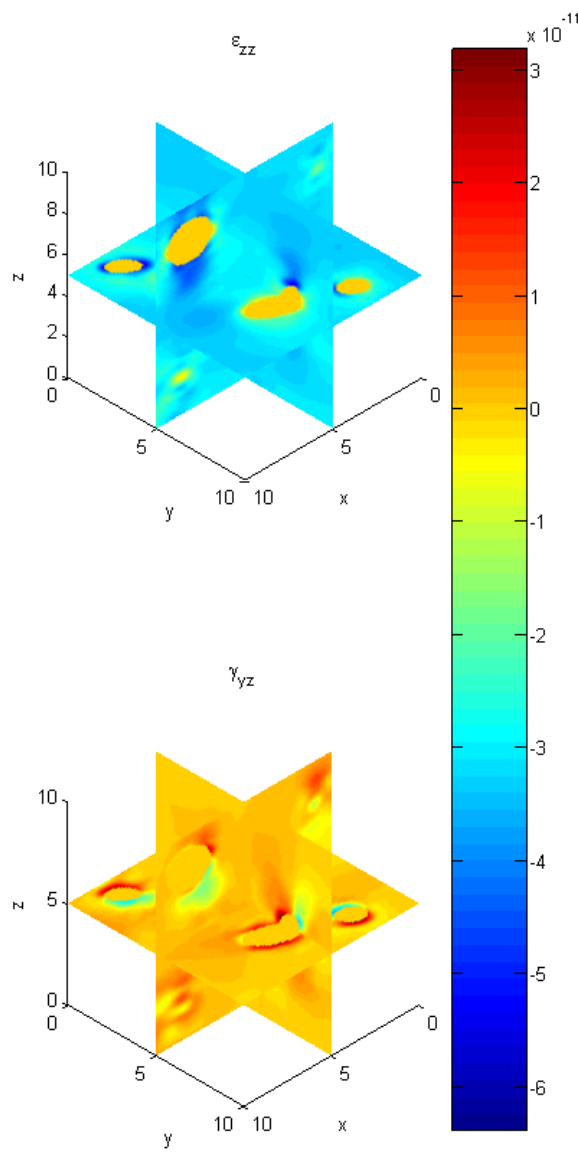

Figura 43 - Distribuição das deformações no modelo com 13 agregados. 

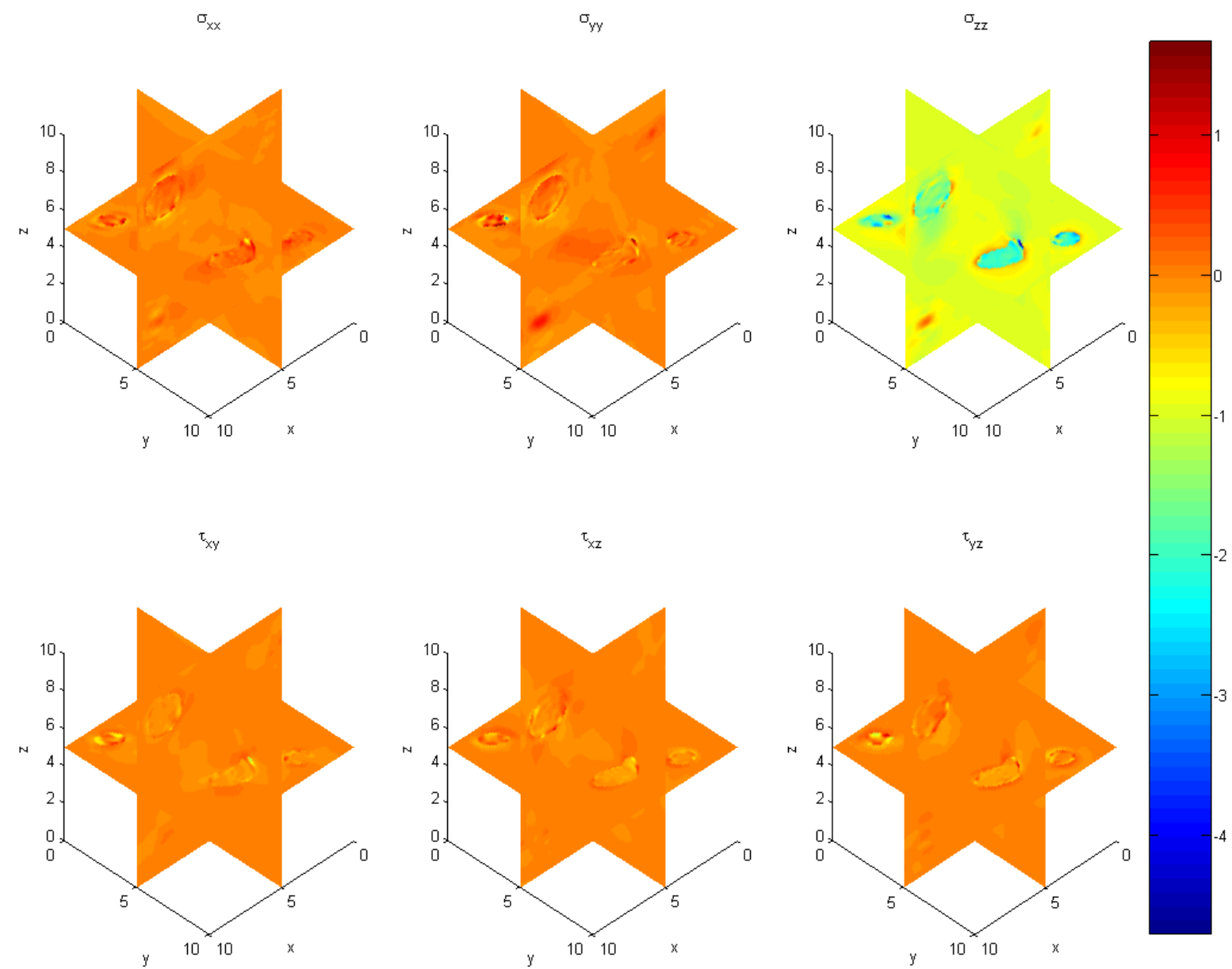

Figura 44 - Distribuição das tensões no modelo com 13 agregados.

\subsection{Homogeneização Dos Modelos Heterogêneos}

Inicialmente, obteveram-se os resultados do modelo com uma inclusão esférica e comparados com os limites de Reuss - Voigt. O valor da rigidez efetiva do material ficou dentro dos limites. Como foi para o modelo com baixa fração de inclusão, o módulo de elasticidade ficou próximo do módulo da argamassa. Neste modelo foi admitido um coeficiente de Poisson igual para as duas fases presentes.

Tabela 7 - Homogeneização das propriedades elásticas do modelo com uma inclusão esférica

\begin{tabular}{|cccc|}
\hline Módulo de Elasticidade & Limite de Reuss & Resultado & Limite de Voigt \\
\hline Coeficiente de Poisson & 0,30 & $33,987 \mathrm{GPa}$ & $353,643 \mathrm{GPa}$ \\
\hline
\end{tabular}


A Tabela 7 e a Figura 38 ilustram os resultados da homogeneização obtida com o modelo com várias inclusões e com diferentes frações de inclusão. Os resultados da rigidez do material nas diversas frações ficaram entre os limites de Reuss - Voigt. Para uma melhor visualização foi fixado uma escala logaritma no gráfico da Figura 45.

Observa-se que o módulo de elasticidade efetivo do material fica próxima do limite em que a matriz se encontra. Se a matriz apresenta uma rigidez inferior a da inclusão o módulo efetivo fica próximo do limite inferior e se a matriz tem uma rigidez superior a da inclusão, o módulo efetivo fica próximo do limite superior.

Tabela 8- Homogeneização das propriedades do modelo com várias inclusões e com diferentes frações de inclusões.

\begin{tabular}{|ccccccccc|}
\hline \multicolumn{7}{c|}{ Módulo de Elásticidade Efetivo (GPa) } \\
$\begin{array}{c}\text { Fração de } \\
\text { Inclusão }\end{array}$ & 0,00 & 10,45 & 19,90 & 30,16 & 69,86 & 80,10 & 89,55 & 100,00 \\
\hline $\begin{array}{c}\text { Limite } \\
\text { de Reuss }\end{array}$ & 30,0 & 33,50 & 37,47 & 42,98 & 99,48 & 150,57 & 285,50 & 5000,0 \\
\hline $\begin{array}{c}\text { Resultado } \\
\text { (Emat < Eagr) }\end{array}$ & 30,0 & 37,06 & 47,27 & 64,46 & & & & \\
\hline $\begin{array}{c}\text { Resultado } \\
\text { (Emat > Eagr) }\end{array}$ & & & & & 2322,2 & 3171,7 & 3941,2 & 5000,0 \\
\hline $\begin{array}{c}\text { Limite } \\
\text { de Voigt }\end{array}$ & 30,0 & 551,5 & 1022,6 & 1532,0 & 3501,2 & 4010,6 & 4481,5 & 5000,0 \\
\hline
\end{tabular}




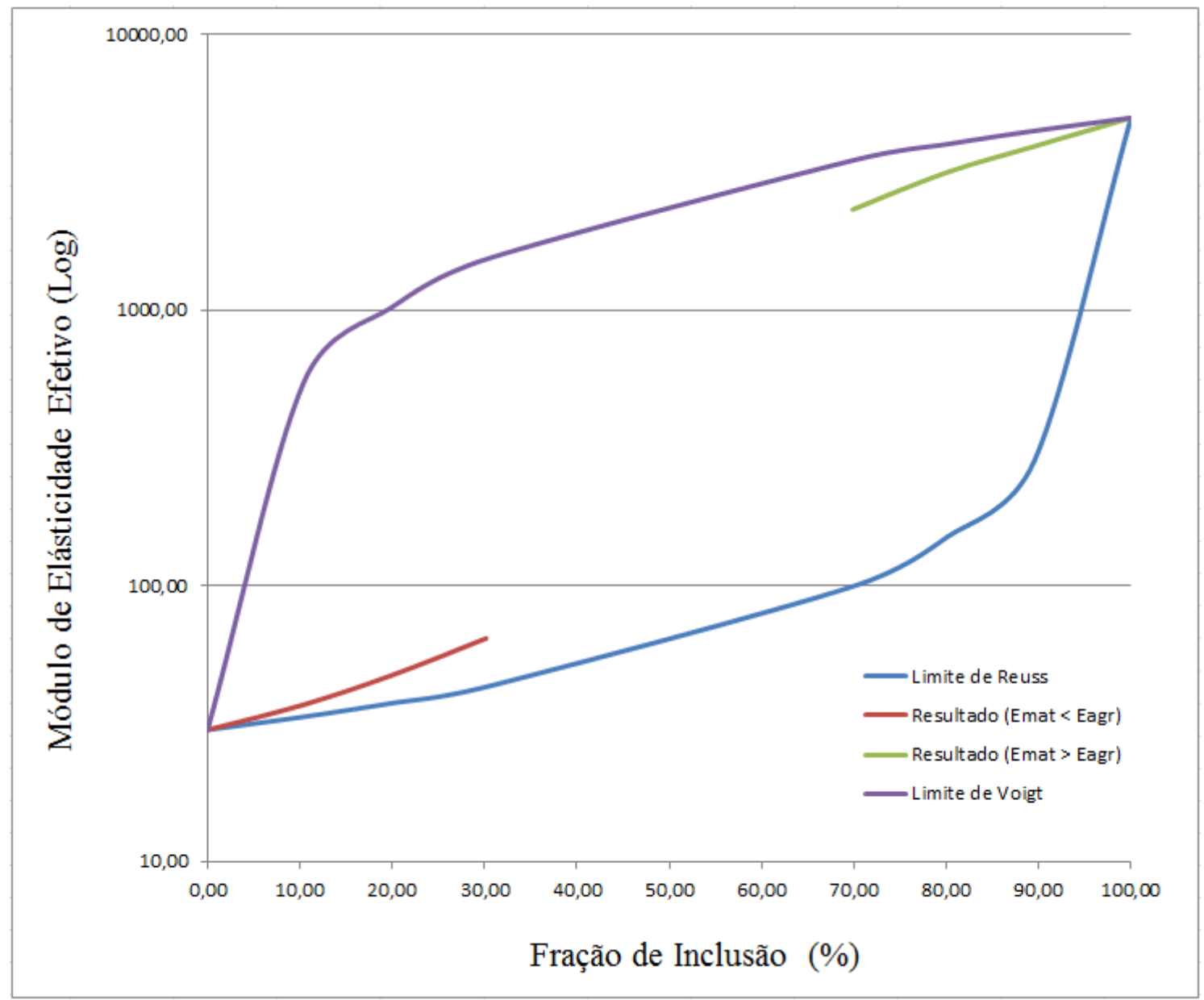

Figura 45 - Curva do módulo de elasticidade do modelo com várias inclusões e diferentes frações de inclusão. 


\section{CONSIDERAÇÕES FINAIS}

A metodologia utilizada nesta pesquisa para simulação computacional da heterogeneidade do concreto com a formulação multiregiões e o método de Galerkin livre de elementos mostrou-se satisfatória. Obtendo-se uma evolução da danificação com uma inclusão coerente com o previsto na literatura e para o caso com várias inclusões, uma região preferencial de ruptura no contorno dos agregados e nas regiões com maior quantidade de inclusões.

Como já relatado no primeiro capítulo, esta pesquisa é o ponto de partida de uma grande linha de pesquisa para a obtenção do envelhecimento do concreto presente nas barragens das usinas hidrelétricas, a partir de modelos computacionais avançados. O objetivo de iniciar esse caminho foi alcançado com a metodologia proposta neste trabalho.

\subsection{Conclusões}

- Foram obtidos resultados satisfatórios na metodologia de multiregiões com o método de Galerkin livre de elementos. Porém, elevando a dificuldade da imposição das fronteiras naturais e essenciais. O mecanismo de definição das fronteiras no MGLE é uma desvantagem quando comparada com o MEF.

- Conseguiu-se uma melhor continuidade dos resultados na interface dos domínios com a utilização do método de Galerkin livre de elementos.

- Observou-se uma melhor convergência das iterações de Newton - Raphson quando admitido a matriz de rigidez danificada, porém mesmo assim encontrando dificuldade na convergência depois de um determinado grau de danificação do material.

- Verificou-se que os resultados da rigidez homogeneizada do concreto quando admitido à argamassa com módulo de elasticidade menor que a do agregado graúdo apresenta resultados que se aproximam do limite inferior. Diferente quando o modulo de elasticidade da argamassa for superior que a do agregado, onde tem resultados que se aproximam do limite superior. Esses 
dois exemplos de material são encontrados na realidade como ilustra a Figura 46.

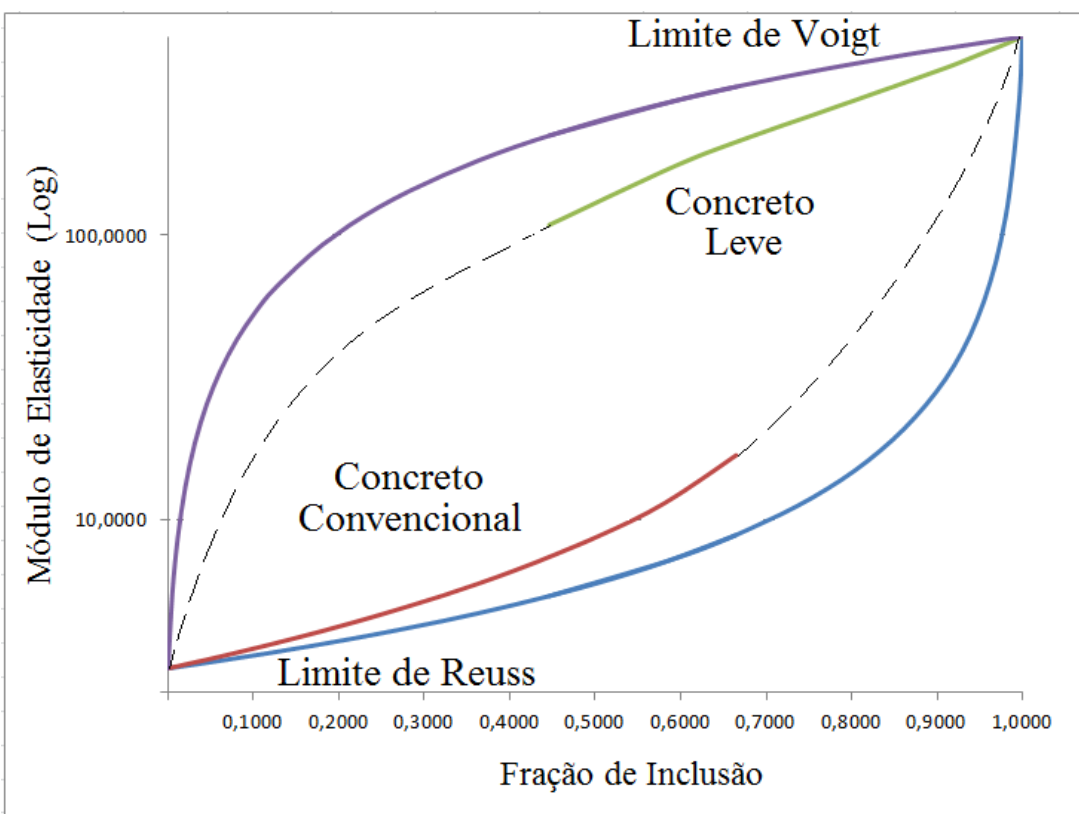

Figura 46 - Comportamento típico da homogeneização da rigidez do concreto.

- Esse comportamento diferente na homogeneização do material da Figura 46 se explica devido à mudança do comportamento do material resultante. Como por exemplo, o caso de concretos leves apresenta ruptura no agregado graúdo e no caso de concreto convencional a ruptura ocorre na argamassa.

- Observou-se danificação do modelo com uma inclusão ao ser submetido ao ensaio de compressão axial coerente com o que encontrado na literatura, apresentando as regiões de ruptura entre a inclusão e os limites do corpo de prova analisado (Figura 47). 


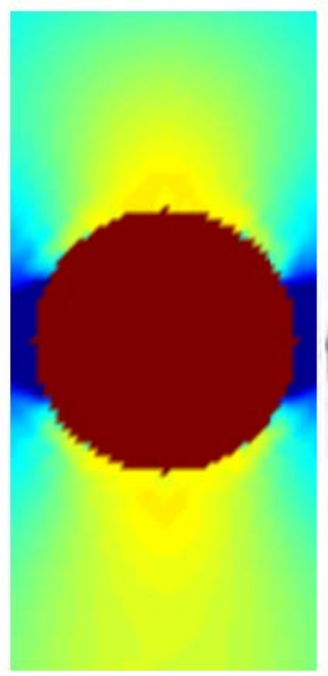

(a)

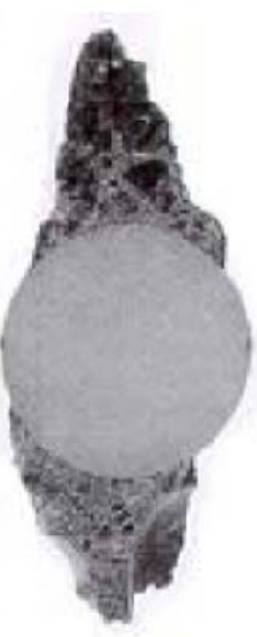

(b)

Figura 47 - (a) Modelo com a região de danificação, (b) Região danificada obtida na literatura [5]

- Obteve-se um mapa da evolução de danificação do modelo computacional com vários agregados satisfatório, apresentando os caminhos preferencias de ruptura na região com maior numero de agregado e no seu contorno (caminho 22). Além disso também ocorreu uma ruptura localizada devido à presença de uma pequena parte de agregado (caminho 11, Figura 48).

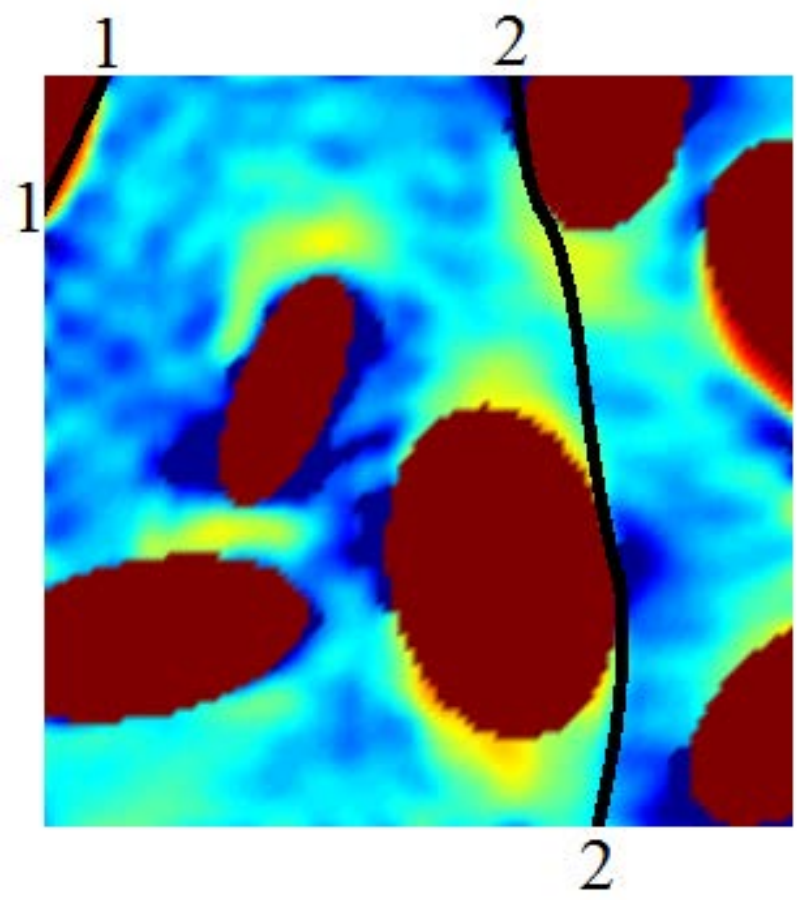

Figura 48 - Caminhos preferencias de ruptura do concreto. 


\subsection{Futuros Desenvolvimentos}

Como recomendações para trabalhos futuros sugerem-se as seguintes pesquisas:

- Desenvolvimento de modelos computacionais da heterogeneidade do concreto, considerando a presença dos vazios e da zona de transição existente entre o agregado graúdo e a argamassa.

- Desenvolvimento de um algoritmo que considere a granulometria do agregado graúdo e a elevada fração de inclusão conforme o encontrado em concretos convencionais.

- Desenvolvimento de um modelo de reação álcali - agregado que simule a evolução dessa deterioração presente em barragens de concreto das usinas hidrelétricas.

- Desenvolvimento do modelo de dano nos modelos computacionais tri dimensionais com adequada fração de inclusão. O modelo de dano deve considerar tanto a evolução da microfissuras com a danificação pela reação álcali - agregado.

- Desenvolvimento de modelos de montantes de barragens a partir de resultados obtidos de modelos meso - mecânicos. Com isso obtendo regiões preferencias de colapso da estrutura.

- Comparação dos resultados obtidos nos modelos computacionais avançados com os ensaios de testemunhos retirados das barragens e com isso obtendo o real estado de conservação dessas grandes obras da Engenharia. 


\section{REFERÊNCIAS BIBLIOGRÁFICAS}

[1] MEHTA, P.K., MONTEIRO, P.J.M. Concreto - Microestrutura, propriedades e materiais. IBRACON, 2008

[2] MONTEIRO, P. J. M. Controle da microestrutura para o desenvolvimento de concretos de alto desempenho. Boletim Técnico. BT/PCC/86. São Paulo. 1993

[3] Drugan WJ,WJR. A micromechanics-based nonlocal constitutive equation and estimates of representative volume element size for elastic composites. J. Mech. Phys. Solids 1996; 44:497-524

[4] SBES. Simulation-Based Engineering Science. Report of the National Science Foundation Blue Ribbon Panel (NSF). 2006

[5] van Mier JGM. Fracture processes of concretes - assessment of material parameters for fracture models, vol. 1. CRC Press : 1997.

[6] QUINTANILLA, J., TORQUATO, S. Microstructure functions for a model of statistically inhomogeneous random media. Physical reviem. 1997; 55

[7] TORQUATO, S. Statistical description of microstructures. Annu. Rev. Mater. Res. 2002; 32:77-111

[8] TORQUATO, S. Random heterogeneous materials. Springer : 2001.

[9] TORQUATO, S. Modeling of physical properties of composite materials. Internacional Journal of Solids and Structures 2000; 37:411-422

[10] GHONIEM, N. M., BUSSO, E. P., KIOUSSIS, N., HUANG, H. Multiscale modeling of nanomechanics and micromechanics: an overview. Philosophical magazine. 2003; 83:3475-3528

[11] GRANVILLE, J., NEVILLE, A. Prediction of concrete durability. E \& FN Spon. 1997 
[12] JIAO, Y., STILLINGER, F. H., TORQUATO, S. Modeling heterogeneous materials via two-point correlation function: algorithmic details. Physical review. 2007; 76

[13] JIAO, Y., STILLINGER, F. H., TORQUATO, S. Modeling heterogeneous materials via two-point correlation function: basic principles. Physical review. 2007; 76

[14] LEE, H. K., SIMUNOVIC, S. A micromechanical constitutive model of progressive crushing in random carbon fiber polymer matrix composites. ORNL/TM-1999/158. 1999

[15] LIU, W. K., MCVEIGH, C. Predictive multiscale theory for design of heterogeneous materials. Computational Mechanic. 2008; 42:147-170

[16] MCVEIGH, C., LIU, W. K. Linking microstructure and properties through a predictive multiresolution continuum. Computer methods in applied mechanics and engineering. 2008

[17] VERNEREY, F. J., LIU, W. K., MORAN, B., OLSON, G. A micromorphic model for the multiple scale failure of heterogeneous materials. Journal of the mechanic and physics of solids. 2008; 56:1320-1347

[18] ZASTRAU, B., LEPENIERS, I., RICHTER, M. On the multi scale modeling of textile reinforced concrete. Technische mechanic. 2008:53-63

[19] Proença SPB,PJJC. A damage constitutive model accounting for induced anisotropy and bimodular elastic response. Latin American Journal of Solids and Structures 2003:101117

[20] Pituba JJC. Método do estado local aplicado na formulação de modelos constitutivos de dano para o concreto. Acta Scientiarum Technology 2009; 31:15-23

[21] Segurado J,LJ. A new three-dimensional interface finite element to simulate fracture in composites. International Journal of Solids and Structures 2004; 41:2977-2993

[22] Santos HC. Análise de estruturas de concreto sob o efeito do tempo: uma abordagem consistente com consideração da viscoelasticidade, da plasticidade, da fissuração, da protensão e de etapas construtivas, Universidade de São Paulo ed, vol. Escola 
Politécnica. Tese de Doutorado : 2006.

[23] Soutis C,BPWR. Multi-scale modelling of composite material systems: the art of predictive damage modelling. Woodhead publishing in Materials : 2005.

[24] Mattei NJ,MMM,HZ. A micromechanical constitutive model for the behavior of concrete. Mechanics of Materials 2007:357-379

[25] Bažant ZP,OBH. Crack band theory for fracture of concrete. Materials and Structures, RILEM 1983:155-177

[26] Dahl KKB. A constitutive model for normal and high-strength concrete.. Department of Structural Engineering, Technical University of Denmark. 1992

[27] ABOUDI, J. Mechanics of composite materials. Studies in applied mechanics 29. Elsevier : 1991.

[28] CHENG, J. Micromechanics of heterogeneous media. Dissertation of Doctorate. University os Connecticint : 1997.

[29] CHENG, J. S., MEHRALEN, S. Multi-scale modeling of heterogeneous materials with fixed and envolving microstructures. Modelling and Simulation in materials science and Engineering. 2005; 13:95-121

[30] LI, S. Introduction to micromechanics and nanomechanics. Lecture notes (CE236/C214). University of California. 2006

[31] NEMAT-NASSER, S., HORI, M. Micromechanics: overall properties of heterogeneous materials. Elsevier : 1999.

[32] PIERARD, O. Micromechanics of inclusion-reinforced composites in elasto-plasticity and elasto-viscoplasticity: modeling and computation. Thèse de Docteur en Sciences Appliquèes. Faculté des Scienses Appliquèes. Université Catholique de Louvain : 2006.

[33] ZOHDI, T., WRIGGERS, P. Introduction to computational micromechanics. Springer : 
2005.

[34] ZOHDI, T., WRIGGERS, P. Modeling and simulation of the decohesion of particulate aggregates in a binging matrix. Engineering Computational. 2001; 95:79-95

[35] Drago A,PMJ. Micro-macromechanical analysis of heterogeneous materials: Macroscopically homogeneous vs periodic microstructures. Composites Science and Technology 2007:1243-1263

[36] Huet C. Coupled Size and Boundary-Condition Effects in Viscoelastic Heterogeneous Composite Bodies. Mech. Mater 1999:787-829.

[37] C. H. Application of variational concepts to size effects in elastic heterogeneous bodies. J. Mech. Phys. Solids 1990:813-841

[38] Hollister SJ,KN. A comparison of homogenization and standard mechanics analyses for periodic porous composites. Comput Mech 1992:73-95

[39] Hollister SJ,KN. Homogenization theory and digital imaging: a basis for studying the mechanics and design principles of bone tissue. Biotechno / Bioengng 1994:586-596

[40] Pecullan S,GLV,TS. Scale effects on the elastic behavior of periodic and hierarchical two-dimensional composites. J. Mech. Phys. Solids 1999:1509-1542

[41] KANIT, T., FOREST, S., GALLIET, I., MOURROURY, V., JEULIN, D. Determination of the size of the representative volume element for randon composites: statistical and numerical approach. International Journal of Solids and Structures. 2003; 40:3647-3679

[42] Velasquez RA. On the representative volume element of asphalt concrete with applications to low temperature. University of Minnesota : Doctoral thesis, 2009.

[43] SWARMINATHAN, S., GHOSH, S., PAGANO, N. J. Statistically equivalent representative volume elements for unidirectional composite microstructures part I without damage. Journal of composite materials 2006; 40 
[44] SWARMINATHAN, S., GHOSH, S., PAGANO, N. J. Statistically equivalent representative volume elements for unidirectional composite microstructures part II with interfacial delonding. Journal of composite materials. 2006; 40

[45] Povirk GL. Incorporation of microstructural information into models of two-phase materials. Acta Metall. Mater 1994:3199-3206

[46] ESHELBY, J. D. Elastic inclusions and inhomogeneities. Progress in solid mechanics. 1962; 2:89-104

[47] ESHELBY, J. D. The determination of the elastic field of an ellipsoidal inclusion, and related problems. Proceedings of the Royal Society. 1957; A241:376-396

[48] ESHELBY, J. D. The elastic field outside an ellipsoidal inclusion. Proceedings of the Royal Society.; A252:561-569

[49] BERRYMAN, J. G. Generalization of Eshelby's formula for a single ellipsoidal elastic inclusion to poroelasticity and thermoelasticity. 79. Physical Review Letters : 1979.

[50] BERRYMAN, J. Generalization of Eshelby's formula for a single ellipsoidal elastic inclusion to poroelasticity and thermoelasticity. Lawrence Livermore National Laboratory. UCRL-JC-126047 : 1997.

[51] BRUNING, M. Eshelby stress tensor in larger strain anisotropic damage mechanics. International Journal of Mechanics Scienses. 2004; 46:1763-1782

[52] CHENG, Z. Q., BATRA, R. C. Exact eshelby tensor for a dynamic circular cylindrical inclusion. Journal of Appied Mechanics. 1999; 66

[53] DUAN, H. L., WAMG, J., HUANG, Z. P., KARCHALOO, B. L. Eshelby formalism for nano-inhomogeneities. Proceedings of the Royal Society. 2005; 461:3335-3353

[54] LAZAR, M., KIRCHNER, H. O. K. The eshelby tensor in nonlocal elasticity and in nonlocal micropolar elasticity. Journal of Mechanics of Materials and Structures. 2006; 1 
[55] LEVIN, V. M. AND TOSTADO, J. M. A. Eshelby's formula for an ellipsoidal elastic inclusion in anisotropic poroelasticity and thermoelasticity. International Journal of Fracture. 2003; 119:79-82

[56] MARKENSCOFF, X. On the shape of the eshelby inclusions. Journal of Elasticity 1998; 49:163-166

[57] PAN, E. Eshelby problem of polygonal inclusions in anisotropic piezo-electric full- and half- planes. Journal of the mechanics and physics of solids. 2004; 52:567-589

[58] RU, C. Q. Eshelby's problem for two-dimensional piezoelectric inclusions of arbitrary shape. The Royal Society. 2000; 456:1051-1068

[59] SAUER, R. A., WANG, G., LI, S. The composite eshelby tensors and their applications to homogenization. Acta Mechanica. 2008; 197:63-69

[60] SHARMA, P., SHARMA, R. On the eshelby's inclusion problem for ellipsoids with nonuniform dilatational Gaussian and exponential eigenstrain. Journal of Applied Mechanics. 2003; 70:418-425

[61] ZHENG, Q. S., ZHAO, Z. H. Green's function and eshelby's fields in couple-stress elasticity. International Journal for Multiscale Computational Engineering. 204; 2:1527

[62] Mori T,TK. Average stress in matrix and average elastic energy of materials with misfitting inclusions. Acta Metal 1973:571-572

[63] Yang CC,HR. Double inclusion model for approximate elastic moduli of concrete material. Cement and Concrete Research 1996:83-91

[64] Castañeda PP. The effective mechanical properties of nonlinear isotropic composites. Department of Mechanical Engineering and Applied Mechanics 2002

[65] Hill R. Elastic properties of reinforced solids: Some theoretical principles. J. Mech. Phys. Solids 1963:357-372 
[66] Kachanov LM. Introduction to Continuum Damage Mechanics. Martinus Nijhoff Publishers, 1986.

[67] Lemaitre J,CJ-L. Mechanics of Solids Materials., 1994.

[68] Hult J. Continuum Damage mechanics., 1988.

[69] Geers MGD. Experimental analysis and computacional modelling of damage and fracture. In Technische Universiteit Eindhoven, thesis D (ed.)., 1997.

[70] Mazars J. A description of micro and macroscale damage of concrete structures. Engrg. Fract. Mech., 1986:729-737

[71] Alvares MS. Contribuição ao estudo e emprego de modelos simplificados de dano e plasticidade para a análise de estruturas de barras em concreto armado. Escola de Engenharia de São Carlos : Tese de Doutorado, 1999.

[72] Du Q,GM,JL. Meshfree, probabilistic determination of point sets and support regions for meshless computing. Computer methods in applied mechanics and engineering. 2002; 191:1349-1366

[73] DUARTE, C. A. A review of some methods to solve partial differencial equations. Technical Report 1995; 06

[74] DUMONT, S., GOUBET, O., HA-DUONG, T., VILLON, P. Meshfree methods and boundary conditions. International Journal for Numerical Methods in Engineering. 2000

[75] GUEDES, C. M. C. F. F. M. Métodos sem malha em problemas de mecânica computacional. Aplicação a processos de enformação plástica. Tese de doutorado. Faculdade de Engenharia da Universidade do Porto. : 2006.

[76] HAO, S., LIU, W. K., CHANG, C. T. Computer implementation of damage models by finite element and meshfree methods. Computer methods in applied mechanics and engineering. 2000; 187:401-440 
[77] HIGHAM, D. J., HIGHAN, N. J. Matlab guide, Second ed. Siam, 2005.

[78] HOBBS, D.W. Expansion of the concrete due to alkali-silica reaction. The Structural Engineer 1984; n 1:26-31

[79] JI-FA, Z., WAN-PU, Z., YAO, Z. A meshfree methods and its applications to elastoplastic problems. Journal of Zhejiang University Science. 2005; 2:148-154

[80] LIU, G. R., GU, Y. T. An introduction meshfree methods and their programming. Springer : 2005.

[81] MIERS, L. S. Formulações não-convencionais de métodos do tipo meshless baseados na equação integral e contorno. Tese de doutorado. Programa de Pós-graduação de Engenharia da Universidade Federal do Rio de Janeiro. : 2007.

[82] BELYTSCHKO, T., ORGAN, D., GERBACH, C. Element free galerkin methods for dynamic fracture in concrete. Computer methods in applied mechanics and engineering. 2000; 187:385-399

[83] DOLBOW, J., BELYTSCHKO, T. Numerical integration of the galerkin weak form in meshfree methods. Computational mechanics. 1999; 23:219-230

[84] DOLBOW, J., BELYTSCHKO, T. An introduction to programming the meshless element free galerkin method. Archives of Computational Methods in Engineering. 1998; 5:207-241

[85] FISH, J., BELYTSCHKO, T. A first course in finite elements. Wiley : 2007.

[86] LIU, W. K., HAO, S., BELYTSCHKO, T., LI, S., CHANG, C. T. Multi-scale methods. International Journal for Numerical Methods in Engineering. 2000; 47:1343-1361

[87] RABCZUK, T., BELYTSCHKO, T. Adaptivity for structured meshfree particle methods in 2D and 3D. International Journal for numerical methods in Engineering. 2005; 63:1559-1582 
[88] LI, S., LIU, W. K. Meshfree and particle methods and their applications. American Society of Mechanical Engineers. 2002; 55

[89] FERNANDES, C. M. T. T. Meshless methods: extending the linear formulation and its generalization to geometically exact structural analysis. Tese de doutorado. Instituto Superior Técnico. Universidade Técnica de Lisboa. : 2007.

[90] FERNANDES, C. M. T. T., LEITÃO, V. M. A. A aproximação GMLS no contexto do EFG: Aplicação a problemas C1. Métodos Computacionais em Engenharia.Portugal. 2004

[91] FERNANDES, C. M. T. T., LEITÃO, V. M. A. Desenvolvimento de uma formulação de galerkin sem elements (EFG) na análise de um problema de dano. VII Congresso de Mecânica Aplicada e Computacional, 2003

[92] FERNANDES, C. M. T. T., PIMENTA, P. M. Uma formulação híbrida para a análise geometricamente exact de cascas e sua implementação sem malha. CILAMCE, 2007

[93] FERNANDES, C. M. T. T., PIMENTA, P. M. An EFG methods for the nonlinear analysis of plates undergoing arbitrarily large deformations. Engineering analysis with boundary elements. 2007

[94] FERNANDES, C. M. T. T., PIMENTA, P. M. Geometrically exact analysis of space frames by a meshless method. ECCOMAS Thematic Conference on Meshless Methods, 2005

[95] LACASTER, P., SALKAUSKAS, K. Surfaces generated by moving least squares methods. Mathematics of Computation. 1981; 155:141-158

[96] WANG, G., LI, S., SAUER, R. A circular inclusion in a finite domain II. The Neumann-Eshelby problem. Acta Mechanica. 2005; 179:91-110

[97] LIU, W. K., KARPOV, E. G., ZHANG, S., PARK, H. S. An introduction to computational nanomechanics and materials. Computer methods in applied mechanics and engineering. 2004; 193:1529-1578 
[98] PIMENTA, P. M., PAULETTI, R.M.O. E PAPPALARDO, A. Uma proposta de metodologia de acompanhamento por modelagem matemática de barragens em concreto afetadas por reação álcali - agregado. Simpósio sobre Reatividade álcali-agregado em Estruturas de Concreto. CBGB/Furnas., 1997

[99] PAPPALARDO, A. Uma metodologia para a modelagem matemática de barragens de concreto afetadas pela reação álcali-agregado. Tese de doutorado. Escola Politécnica da Universidade de São Paulo. : 1998.

[100] BORDAS, S., RABCZUK, T., ZI, G.:. Three-dimensional crack initiation, propagation, branching and junction in non-linear materials by an extended meshfree method without asymptotic enrichment. Engineering Fracture Mechanics. 2008; 78:943-960

[101] LI, S., SAUER, R., WANG, G. The eshelby tensors in a finite spherical domain - part II: applications to homogenization. Journal of Applied Mechanics. 2007; 74:784-797

[102] LI, S., SAUER, R., WANG, G. The eshelby tensors in a finite spherical domain - part I: theoretical formulations. Journal of Applied Mechanics. 2007; 74:770-783

[103] CHATELLIER, L. The Constitution of Hydraulic Mortars. Mcgraw Hill : New York, 1905.

[104] DENT-GLASSER, L.S., KATAOKA, N. The chemistry of alkali-aggregate reaction. Cement and Concrete Research 1981; 11:1-9

[105] SIMS, I. The assessment of concrete for ASR. Concrete. 1992; 26:42-46

[106] WANG, G., LIU, X., LI, S., SITAR, N. Smart element method II: An element based on the finite eshelby tensor. International Journal for Numerical Methods in Engineering. 2005; 65:1303-1333

[107] PAULON, V.A. Reações álcali-agregado em concreto. Dissertação de mestrado. Escola Politécnica da Universidade de São Paulo. : 1981.

[108] SILVA, P. N. E KUPEIMAN, S. C. Reação álcali-agregado nas usinas hidroelétricas do complexo Paulo Afonso /CHESP. Boletim Técnico da Escola Politécnica da 
Universidade de São Paulo. BT/PCC/480. 2008

[109] NEVILLE, A. M. Propriedades do Concreto, 2ª ed. Editora PINI. : 1997.

[110] PIETRUSZCZAK, S. On the mechanical behavior of concrete subjected to alkaliaggregate reaction. Computers and Structures. 1996; 58:1093-1097

[111] TANAKA, K., MORI, T. Note on volume integrals of the elastic field around an ellipsoidal inclusion. Journal of Elasticity. 1972; 2:199-200

[112] KASSNER, M. E., NEMAT-NASSER, S., SUO, Z., BAO, G., BARBOUR, J. C., BRINSON, L. C., ESPINOSA, H., GAO, H., GRANICK, S., GUMBSCH, P., KIM, K., KNAUSS, W., KUBIN, L., LANGER, J., LARSON, B. C., MAHADEVAN, L., MAJUNDAR, A., TORQUATO, S., SWOL, F. New directions in mechanics. Mechanics of materials. 2005; 37:231-259

[113] LIANG, Z. Modelling of stiffness degradation in materials with micromechanical and nanomechanical effects. Dissertation of Doctorate. University of Miami. : 2005.

[114] LOPES, L. E. Modelagem mecânica e numérica da reação álcali-agregado com aplicação a barragens de concreto. Tese de doutorado. Programa de Pós-graduação de Engenharia da Universidade Federal do Rio de Janeiro. : 2004.

[115] CHAMILCO, G. E. I. Modelagem computacional micromecanica de poroviscoelasticidade. Tese de doutorado. Laboratório Nacional de Computação Cientifica. : 2006.

[116] KIN, H. M., PAULINO, G. H., BUTTLAR, W. G., SUN, L. Z. Micromechanics based thermoelastic model for functionally graded particulate materials with particle interactions. Journal of the mechanics and physics of solids. 2007; 55:132-160

[117] JU, J. W., KO, Y. F. Micromechanical elastoplastic damage modeling of progressive interfacial arc debonding for fiber reinforced composites. International Journal of Damage Mechanics. 2008

[118] MORANVILLE-REGOURD, M. Microestruture of high performance concrete. High 
performance concrete - from material to structure.:3-13

[119] BARROS, F. B. Métodos sem malha e método dos elementos finitos generalizados em análise não-linear de estruturas. Tese de doutorado. Escola de Engenharia de São Carlos da Universidade de São Paulo : 2002.

[120] LI, S., LIU, W. K. Meshfree particle methods. Springer : 2004.

[121] MENDEZ, S. F. Meshfree methods and finite elements: friend or foe? Doctoral thesis. Facultat de matematigues i estadistica. Universitat politécnica de catalunya. : 2001.

[122] BÉRARD, J., ROUX, R. La viabilité des bétons du Québec: le role desgranulats. Canadian Journal of Civil Engineering. 1986; 13:12-24

[123] ULM, F. H., CONSTANTINIDES, G., HEUKAMP, F. H. In concrete a poromechanics materials? A multiscale investigation of poroelastic properties. Materials and Structures. 2004; 37:43-58

[124] BISWAS, A., SHAPIRO, V., TSUKANOV, I. Heterogeneous materials modeling with distance fields. Computer aided geometric design. 2004; 21:215-242

[125] ZEMAM, J., SEJNOHA, M. From random microstructures to representative volume elements. Modelling and Simulation in Materials Science and Engineering. 2007; 15:325-335

[126] BAYARD, O., PLÉ, O. Fracture mechanics of reactive powder concrete: material modeling and experimental investigations. Engineering Fracture Mechanics. 2003; 70:839-851

[127] ROESLER, J., PAUlino, G. H., GAEDICKe, C., BORDELON, A., PARK, K. Fracture behavior of functionally graded concrete materials for rigid pavements. Transportation Research record. 2007

[128] KIN, H. M., PAULINO, G. H., BUTTLAR, W. G., SUN, L. Z. Effective thermal conductivity of functionally graded particulate nanocomposites with interfacial thermal resistance. Journal of Applied Mechanics. 2008; 75 
[129] LEMARCHAND, E. Contribution de La Micromécanique à l'étude des phénomènes de transport ET couplage poromécanique dans lês milieux poreux: Application aux. phénomènes de gonglement des géomatériaux. Thèse de Doctorat. École Nationale des Ponts ET Chausses. Paris. France : 2001.

[130] DAL MOLIN, D. C. C. Contribuição ao estudo das propriedades mecânicas dos concretos de alta resistência com e sem adições de microsilica. Tese de Doutorado. Escola Politécnica da Universidade de São Paulo. : 1995.

[131] SWAMI, R. N. Alkali-silica reaction an concrete structures. Structural Engineering Review 1990; 2:89-103

[132] LEPS, T. M. Alkali-aggregate reaction (AAR) - a brief history of its basic chemistry. Internacional Conference on Concrete Alkali-Aggregate Reactions in Hydroelectric Plants and Dams. USCOLD, 1995

[133] PEDERSEN, B. M. Alkali- reactive and inert fillers in concrete. Dissertation of Doctorate. Faculty of Engineering Science and Technology. Norwegian University of Science and Technology. : 2004.

[134] SILVA, V. M. Ação da carbonatação em vigas de concreto armado em serviços, construídas em escala natural. Tese de doutorado. Escola de Engenharia de São Carlos da Universidade de São Paulo. : 2007.

[135] ONAKA, S. A relationship among the components of the eshelby tensor and its physical meaning in general inlusions problems. Philosophical magazine letters. 2000; 80:367-370

[136] LI, S., SAUER, R., WANG, G. A circular inclusion in a finite domain I. The DiricheletEshelby problem. Acta Mechanica. 2005; 179:67-90 